Gilles Pierre Loïc Thomas

Modeling, Design and Manufacturing of an Acoustic Levitation Linear Transportation System 
Gilles Pierre Loïc Thomas

\section{Modeling, Design and Manufacturing of an Acoustic Levitation Linear Transportation System}

Dissertação apresentada à Escola Politécnica da Universidade de São Paulo para obtenção do Título de Mestre em Engenharia Mecânica. 
Gilles Pierre Loïc Thomas

\section{Modeling, Design and Manufacturing of an Acoustic Levitation Linear Transportation System}

Dissertação apresentada à Escola Politécnica da Universidade de São Paulo para obtenção do Título de Mestre em Engenharia Mecânica.

Área de concentração:

Engenharia de Controle e Automação Mecânica

Orientador:

Prof. Dr. Emílio Carlos Nelli Silva 
Ficha Catalográfica

Thomas, Gilles Pierre Loïc

Modeling, Design and Manufacturing of an Acoustic Levitation Linear Transportation System. São Paulo, 2015. 101 p.

Dissertação (Mestrado) — Escola Politécnica da Universidade de São Paulo. Departamento de Engenharia Mecatrônica e de Sistemas Mecânicos.

1. Levitação acústica. 2. Ultrassom. I. Universidade de São Paulo. Escola Politécnica. Departamento de Engenharia Mecatrônica e de Sistemas Mecânicos. II. Título. 


\section{Abstract}

Acoustic levitation is a method which uses sound radiation to suspend matter in a medium. The main use of this phenomenon is for the contactless processing of matter, allowing to manipulate small objects without any solid contact. Contactless processing of matter presents many advantages in, for example, the fabrication of MEMS (microelectromechanical systems) where handling the components is challenging because of their fragile and surface-sensitive characteristics or in the chemical/biological industry when handling high-purity or hazardous materials. Thus, a new device for noncontact linear transportation of small solid objects is presented here. In this device, ultrasonic flexural vibrations are generated along the ring shaped vibrator using two Langevin transducers and by using a reflector parallel to the vibrator, small particles are trapped at the nodal points of the resulting acoustic standing wave. The particles are then moved by generating a traveling wave along the vibrator, which can be done by modulating the vibration amplitude of the transducers. The working principle of the traveling wave along the vibrator has been modeled by the superposition of two orthogonal standing waves, and the position of the particles can be predicted by using finite element analysis of the vibrator and the resulting acoustic field. A prototype consisting of a $3 \mathrm{~mm}$ thick, $220 \mathrm{~mm}$ long, $50 \mathrm{~mm}$ wide and $52 \mathrm{~mm}$ radius aluminum ring-type vibrator and a reflector of the same length and width was built and small polystyrene spheres have been successfully transported along the straight parts of the vibrator. 


\section{Resumo}

Levitação acústica é um método para suspender matéria em um meio através de pressão de radiação acústica gerada por intensas ondas de som. O principal uso desse fenômeno é na manipulação de partículas sem contato solido. Esse fenômeno tem várias aplicações para pesquisas onde deve ser evitado todo o contato como, por exemplo, na área de biologia, química, e na fabricação de MEMS. Assim, um novo sistema de transporte linear de partículas por levitaç acústica está apresentado aqui. Nesse sistema, vibrações flexurais estão geradas em uma placa tipo anel com dois transdutores tipo Langevin, e colocando um refletor paralelo ao oscilador, partículas estão presas no pontos nodais da onda acústica gerada. As partículas estão deslocadas modulando a amplitude dos transdutores. Assim, este trabalho tem como objetivos a modelagem do fenômeno de levitação acústica, o dimensionamento de um protótipo de sistema de transporte linear de partículas por levitação acústica, bem como a fabricação e o controle desse protótipo. Um protótipo consistindo de uma estrutura tipo anel de alumínio de $3 \mathrm{~mm}$ de espessura, $220 \mathrm{~mm}$ de comprimento e um raio de $52 \mathrm{~mm}$ foi fabricado e o transporte de pequenas esferas de isopor foi realizado com exito nas parte retas do vibrador. 


\section{Contents}

\section{List of Figures}

\section{List of Tables}

\section{List of Symbols}

1 Introduction 1

1.1 Background ............................. 1

1.2 Acoustic levitation phenomenon . . . . . . . . . . . . . . . 2

1.3 Single-axis acoustic levitator . . . . . . . . . . . . . . . . . 3

1.4 Acoustic levitation transportation systems . . . . . . . . . . . . . . . 4

1.5 Other applications of the acoustic levitation . . . . . . . . . . . . 6

1.6 Motivation of study . . . . . . . . . . . . . . . . . . . . . . 7

1.7 Objectives . . . . . . . . . . . . . . . . . . 8

1.8 Dissertation overview . . . . . . . . . . . . . . . . . . . . . . . . . . . . . . 8

2 Theory of acoustic levitation 9

2.1 Acoustic plane waves . . . . . . . . . . . . . . . . . 9

2.2 Acoustic radiation force: Gor'kov theory . . . . . . . . . . . . . . . . . 10

2.3 Acoustic transducers . . . . . . . . . . . . . . . . . . . 11

2.3 .1 Piezoelectricity . . . . . . . . . . . . . . . 11

2.3.2 Langevin transducer coupled with mechanical amplifier . . . . . . . 12

2.3.3 Acoustical horn . . . . . . . . . . . . . . . . . 12

3 Theoretical modeling of the ring-type vibrator $\quad 14$ 
3.1 Working principle . . . . . . . . . . . . . . . . . 14

3.2 Analytical modeling of a ring-type structure . . . . . . . . . . . . 17

3.2 .1 Straight part . . . . . . . . . . . . . . 17

3.2 .2 Curved part . . . . . . . . . . . . . . . . . . 18

3.2.3 Boundary conditions . . . . . . . . . . . . . . . . 20

3.2.4 Solutions . . . . . . . . . . . . . . . . . . . 20

3.2 .5 Iterative design . . . . . . . . . . . . . . . . . . 24

4 Numerical modeling $\quad 26$

4.1 Finite element modeling of the beam vibrator . . . . . . . . . . 26

4.1.1 Finite element formulation . . . . . . . . . . . . . . 26

4.1 .2 Modal analysis . . . . . . . . . . . . . . . . . 30

4.1 .3 Harmonic analysis . . . . . . . . . . . . . . . . . . . 30

4.1.4 MatLab implementation .................. 31

4.2 Finite element modeling of the ring-type vibrator . . . . . . . . . . 31

4.3 Finite element modeling of the acoustical horns . . . . . . . . . . . . 32

4.4 Finite element modeling of the acoustic transducers . . . . . . . . . . 33

4.5 Modeling of the acoustic field . . . . . . . . . . . . . 34

$4.5 .12 \mathrm{D}$ model . . . . . . . . . . . . . . . . . 34

$4.5 .23 \mathrm{D}$ model . . . . . . . . . . . . . . . . . . . 40

4.6 Finite element iterative design by using ANSYS and MatLab . . . . . . . . 41

5 Design of the prototype $\quad 42$

5.1 Introduction . . . . . . . . . . . . . . . . . . 42

5.2 Length, thickness and material of the vibrator . . . . . . . . . . . . 42

5.3 Width of the vibrator . . . . . . . . . . . . . . 43

5.4 Radius of the vibrator . . . . . . . . . . . . . . . . . 44

5.5 Design of the acoustical horns . . . . . . . . . . . . . 47

5.6 Design of the transducers . . . . . . . . . . . . . . . . . 49 
6.1 Beam vibrator prototype . . . . . . . . . . . . . . 51

6.1.1 Analysis of the vibrating plate . . . . . . . . . . . . . 51

6.1.2 Analysis of the acoustic field . . . . . . . . . . . . 55

6.2 Ring-type vibrator prototype f . . . . . . . . . . . . . . 58

6.2.1 Analysis of the ring-type vibrator . . . . . . . . . . . . 58

6.2.2 Analysis of the resulting acoustic field . . . . . . . . . . . . 60

7 Manufacturing of the prototype $\quad 62$

7.1 Introduction . . . . . . . . . . . . . . . . . . . . 62

7.2 Bending and welding a plate . . . . . . . . . . . . . . 62

7.3 Full machining using a CNC mill . . . . . . . . . . . . . . . . . 63

7.4 Full machining using a CNC wire-cut EDM machine . . . . . . . . . . . . 63

7.5 Manufacturing . . . . . . . . . . . . . . . . . . . . . 64

8 Experimental evaluation $\quad 66$

8.1 Introduction . . . . . . . . . . . . . . . . 66

8.2 Setup . . . . . . . . . . . . . . . . . 66

8.2.1 Acoustic levitation transportation of particle . . . . . . . . . . 66

8.2 .2 Vibration measurement . . . . . . . . . . . . . . . . . . 68

8.3 Results . . . . . . . . . . . . . . . . . . . . 69

8.3.1 Acoustic levitation transportation of particle . . . . . . . . . 69

8.3.2 Vibration measurement . . . . . . . . . . . . . . . . . . 71

9 Conclusion and future work $\quad 73$

$\begin{array}{ll}\text { References } & 75\end{array}$

$\begin{array}{lr}\text { Appendix A } & 79\end{array}$

A.1 MatLab implementation . . . . . . . . . . . . . . . . . . . 79 
A.1.1 Program structure f . . . . . . . . . . . . . . . . . . 79

A.1.2 PMLs boundaries calibration . . . . . . . . . . . . . . 83

A.1.3 Analytical comparison of the beam vibration . . . . . . . . . . 84

Appendix B

B.1 Hamilton's principle applied to the Timoshenko straight beam theory . . . 85

B.2 Hamilton's principle applied to the Timoshenko curved beam theory . . . . 87

B.3 MatLab program for iterative design with the analytical model of the ring-type vibrator . . . . . . . . . . . . . . . . . 89

$\begin{array}{lr}\text { Appendix C } & 93\end{array}$

C.1 Script for acoustic levitation transportation . . . . . . . . . . . . 93

C.2 Script for vibration measurements . . . . . . . . . . . . . . . . 97 


\section{List of Figures}

1.1 Examples of acoustic levitation applications: (a) Raman spectroscopy (PUSKAR et al., 2007) \& (b) Levitating ant (XIE et al., 2006). . . . . . . . . 2

1.2 Principle of acoustic levitation . . . . . . . . . . . . . . . . 3

1.3 Axisymmetric model of a single-axis acoustic levitator from (ANDRADE,

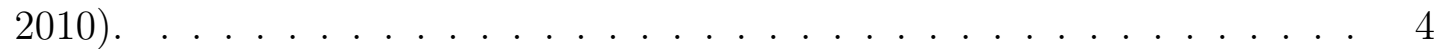

1.4 Linear acoustic levitation transportation system from (KOYAMA;

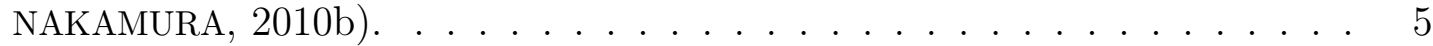

1.5 Circular acoustic levitation transportation system from (KOYAMA;

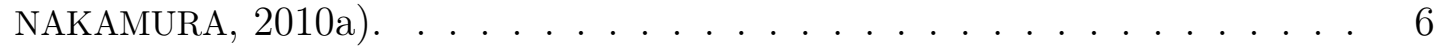

1.6 Contactless mixing of droplets and particles (FORESTI; NABAVI, 2013). $\quad$. . 7

2.1 a/ Levitating particle; b/ dimensionless levitation potential (ANDRADE, 2010). . . . . . . . . . . . . . . . . . . . 10

2.2 Parameterized schematic of a Langevin transducer coupled with a mechanical amplifer. . . . . . . . . . . . . . . . . . . . 12

2.3 The usual profiles for an acoustical horn. . . . . . . . . . . . . . . . 13

3.1 Left: ring-type vibrator, right: frequency difference between the two orthogonal modes as presented by (SEEMANN, 1996). . . . . . . . . . 15

3.2 Polarization of the piezoceramics on the stator (SEEMANN, 1996). . . . . . 15

3.3 Experimental setup of the ultrasonic motor (SEEMANN, 1996). . . . . . . . 15

3.4 Ring-type vibrator. . . . . . . . . . . . . . . . . . . . . 16

3.5 Deformation of a Timoshenko beam. . . . . . . . . . . . . . . . . 17

3.6 Deformation of a curved Timoshenko beam. . . . . . . . . . . . . . . . 19

3.7 Plot of the functions $g_{1}(\omega)$ and $g_{2}(\omega) \ldots \ldots \ldots . \ldots \ldots$

3.8 Plot of the solution 1 and solution 2. . . . . . . . . . . . . 25 
4.1 (a) Acoustic levitation transportation system as presented in (KOYAMA; NAKAMURA, 2010b) (b) Beam model of the vibrating plate. . . . . . . . . 27

4.2 Forces and moment at a point of the beam. . . . . . . . . . . . 27

4.31 1D element of a beam. . . . . . . . . . . . . . . . . . . 28

4.4 ANSYS element SOLID185. . . . . . . . . . . . . . . . . 32

4.5 3D solid model of the ring type structure. . . . . . . . . . . . . 32

4.6 3D solid model of the horns (left: conical profile horn; right: stepped profile horn). . . . . . . . . . . . . . . . . 33

4.7 ANSYS element SOLID226. . . . . . . . . . . . . . . . . 33

$4.8 \mathrm{a} / 3 \mathrm{D}$ solid model of the Langevin transducer (piezoelectric ceramics in purple); b/3D solid model of mechanical amplifier; $\mathrm{c} / 3 \mathrm{D}$ solid model of the complete acoustic transducer (piezoelectric ceramics in purple). . . . . . . 34

4.9 Model of the acoustic field. . . . . . . . . . . . . . . . . 35

4.10 Four-node quad element. . . . . . . . . . . . . . . . . . 36

4.11 Model of the acoustic field with the PML layers. . . . . . . . . . . . . . 39

4.12 3D model of the width analysis. . . . . . . . . . . . . . . . . 40

4.13 ANSYS acoustic element FLUID30 . . . . . . . . . . . . . . . . 41

5.1 Pressure plot of the model. . . . . . . . . . . . . . . . . . 43

5.2 Levitation potential minimum as a function of the width of the plate. . . . 44

5.3 Analytical analysis of the frequency of both solutions for the $20^{\text {th }}$ flexural mode.

5.4 Analytical analysis of the frequency of both solutions for the $22^{\text {nd }}$ flexural mode.

5.5 Analytical analysis of the frequency of both solutions for the $24^{\text {th }}$ flexural mode.

5.6 Comparison between the analytical analysis and the finite elements analysis for the $22^{n d}$ flexural mode. . . . . . . . . . . . . . . . . . . . . . . . . . 46

5.7 Parameters of the acoustical horns (left: conical profile horn; right: stepped profile horn). 
5.8 The four modes of the conical horn with frequencies close to the working frequency of the prototype. . . . . . . . . . . . . . . . . . 48

5.9 The four modes of the stepped horn with frequencies close to the working frequency of the prototype. . . . . . . . . . . . . . . . . . 48

6.1 Relative amplitude of vibration along the beam for some driving phase difference. . . . . . . . . . . . . . . . . . . . 5 52

6.2 Visualization of the vibration along the plate for a complete period of time

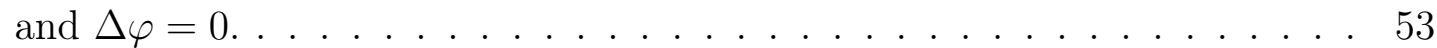

6.3 Visualization of the vibration amplitude along the plate for $\varphi_{1}=0$ and

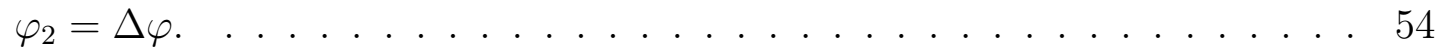

6.4 Translation of the standing wave in function of the driving phase difference 54

6.5 Normalized pressure amplitude, velocity amplitude and dimensionless Gor'kov acoustic levitation potential for $\Delta \varphi=0 . \ldots \ldots$. . . . . . 55

6.6 Normalized dimensionless Gor'kov acoustic levitation potential for $\Delta \varphi=0$ at the extremities. . . . . . . . . . . . . . . . 56

6.7 Relative dimensionless Gor'kov acoustic levitation potential for $\Delta \varphi \in[0,2 \pi]$. 57

6.8 Relative vibration amplitude along the top straight part of the vibrator. . . 59

6.9 Relative vibration amplitude along the bottom straight part of the vibrator. 59

6.10 Visualization of the vibration amplitude along the top straight beam of the vibrator for $\theta \in[0, \pi] \ldots \ldots \ldots \ldots$. . . . . . . . . . . . . . . . 59

6.11 Visualization of the vibration amplitude along the bottom straight beam of the vibrator for $\theta \in[0, \pi] \ldots \ldots \ldots \ldots$

6.12 Relative vibration amplitude along the top straight part of the vibrator. . . 60

6.13 Relative dimensionless Gor'kov acoustic levitation potential for $\Delta \theta \in[0,2 \pi]$. 61

7.1 Estimated uniform pressure cone (BUDYNAS-NISBETT, 2008) . . . . . . . 62

7.2 The radius at the intersection between the horn and the ring-type structure due to the milling process. . . . . . . . . . . . . . . . . . . 63

7.3 Isometric view of the CAD of the final prototype. . . . . . . . . . 64

7.4 Photo of the prototype during the milling. . . . . . . . . . . . . . . . . 64

7.5 Photo of the complete prototype. . . . . . . . . . . . . 65 
8.1 Experimental setup of the prototype for acoustic levitation transportation. 66

8.2 Experimental setup for the vibration amplitude measurement. . . . . . . . 68

8.3 Left: measurement of the vibration amplitude for the top side of the vibrator; Right: measurement of the vibration amplitude for the bottom side of the vibrator. . . . . . . . . . . . . . . . . . . . 69

8.4 Trajectory of the levitated particle. . . . . . . . . . . . . 70

8.5 Distance traveled as a function of $\theta \ldots \ldots \ldots \ldots$. . . . . . . . 71

8.6 Amplitude of the standing wave at the bottom of the vibrator for $\theta \in[0, \pi]$ measured by the laser vibrometer. . . . . . . . . . . . . . . . 71

8.7 Amplitude of the standing wave at the top of the vibrator for $\theta \in[0, \pi]$ measured by the laser vibrometer. . . . . . . . . . . . . . . . . . 72

A.1 Composition of the two main struct variables of the FEM. . . . . . . . . 79

A.2 Steps of a complete analysis of the model fig. 4.9 . . . . . . . . . . . 80

A.3 An example of a mesh generated by this function. . . . . . . . . . . . . . 81

A.4 Model used in this section. . . . . . . . . . . . . . . . . 83

A.5 Normalized pressure amplitude with well scaled PMLs boundaries. . . . . . 83

A.6 Comparison between the analytical solution and FEM solution for the frequency ............................. 84 


\section{List of Tables}

2.1 Matrix variables used in the piezoelectric constitutive equations . . . . . . 11

5.1 Aluminium 5052 physical properties. . . . . . . . . . . . . . . . 42

5.2 Air acoustical properties. . . . . . . . . . . . . . . . . . 43

5.3 Results of the analytical analysis. . . . . . . . . . . . . . . . 46

5.4 Parameters of the designed horns. . . . . . . . . . . . . . . . . . . 48

5.5 Frequencies of the mode of the conical horn close to the working frequency of the prototype. . . . . . . . . . . . . . . . . . . . . . . 48

5.6 Frequencies of the mode of the stepped horn close to the working frequency of the prototype. . . . . . . . . . . . . . . . . . . 48

5.7 PZT4 properties. . . . . . . . . . . . . . . 50

5.8 Parameters of the designed transducer. . . . . . . . . . . . . . . . 50

6.1 Properties of the vibrating plate designed in (KOYAMA; NAKAMURA, 2010a) 51

6.2 Modal analysis of the vibrating plate designed in (KOYAMA; NAKAMURA,

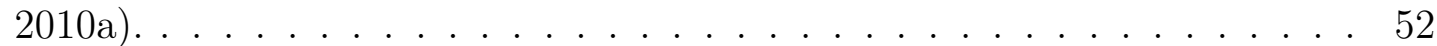




\section{List of Symbols}

$p$ acoustic pressure

$t$ time

$c$ velocity of propagation of the wave

$U$ levitation potential (Gor'kov)

$\tilde{U}$ dimensionless levitation potential

$\rho$ density

$\rho_{f}$ density of the fluid

$\lambda$ wavelength

$\lambda_{a}$ wavelength in the fluid

$\lambda_{v}$ wavelength in the vibrator

$v$ transverse deflection of the beam

$\varphi$ temporal phase of the transducer

$\theta$ spatial phase of the vibrator standing wave 


\section{Introduction}

\subsection{Background}

Acoustic levitation, also called acoustophoresis, is a method which uses sound radiation, usually at the ultrasonic frequency (superior to $20 \mathrm{kHz}$ ), to suspend matter in a medium. The main use of this phenomenon is for the contactless processing of matter, allowing the manipulation almost all substances, liquids, solids and even small living animals (XIE et al., 2006) (fig. 1.1b), without any contamination and contact noise. One of the major advantage that makes acoustic levitation suitable for a wide range of applications lies in the fact that any kind of material can be levitated, in opposition to other methods such as magnetic and electromagnetic levitation that can only work with magnetic materials, and electrostatic levitation that can only work with conductive materials, and so on.

Contactless processing of matter presents many advantages in, for example, the fabrication of MEMS (microelectromechanical systems) where handling the components is challenging because of their fragile and surface-sensitive characteristics (REINHART; HOEPPNER, 2000), or in the chemical/biological industry when handling high-purity or hazardous materials (SANTESSON; NILSSON, 2004). Today, acoustic levitation devices are able to levitate the heaviest solid (iridium, $\rho=22.6 \mathrm{~g} / \mathrm{cm}^{3}$ ) and liquid (mercury, $\rho=13.6 \mathrm{~g} / \mathrm{cm}^{3}$ ) on earth (XIE et al., 2002).

For now, most of the applications are in the chemical/biological research domain, including investigation of the basic physical properties of liquid or solid samples in titration and crystallization experiments, melting and solidification, the dynamics of growth and formation of ice particles, the properties of molten materials, the equilibrium shape and stability of liquid drops, evaporation, the generation and characterization of organic surface layers at the air-water interface and Raman spectroscopy (PUSKAR et al., 2007) (fig. 1.1a). Nowadays, most of the publications on acoustic levitation are working toward applications of the phenomenon in the pharmaceutical industry, in order to produce high purity products (LIU; HU, 2009). 

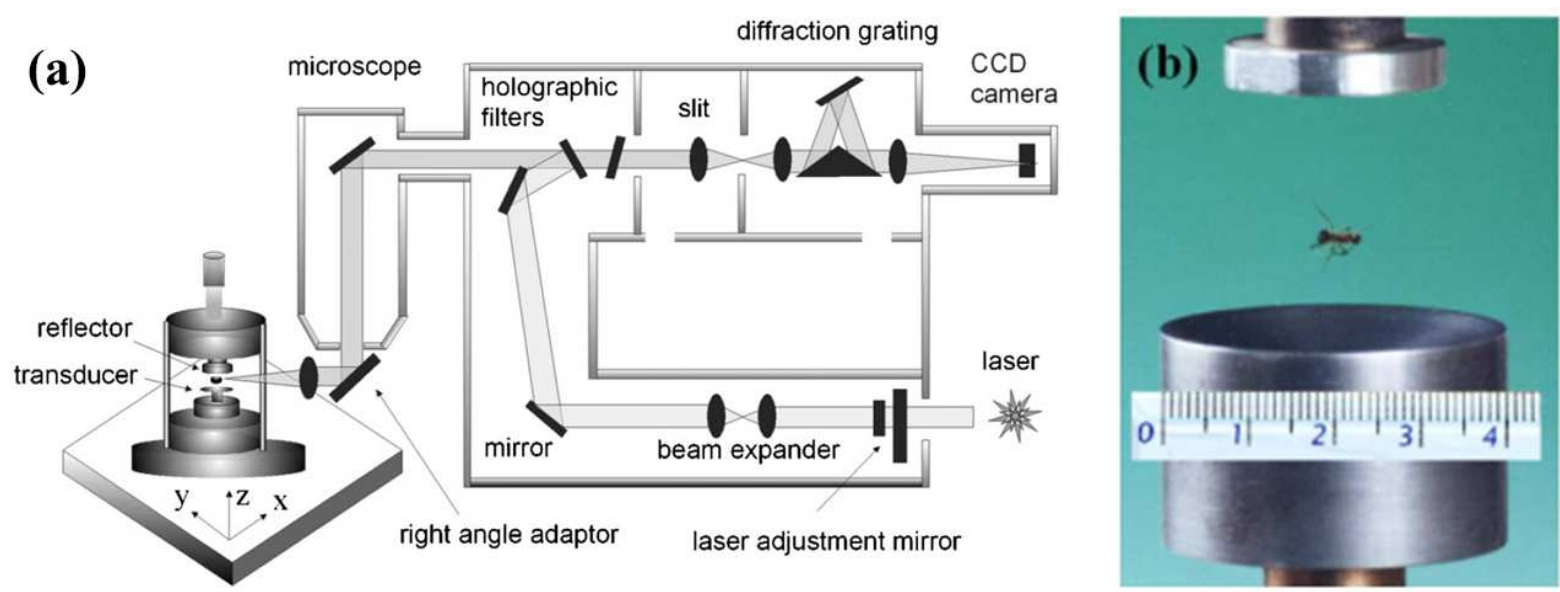

Figure 1.1: Examples of acoustic levitation applications: (a) Raman spectroscopy (PUSKAR et al., 2007) \& (b) Levitating ant (XIE et al., 2006).

The first theoretical work on acoustic levitation goes back to the 1930s, with the work of King on the force on disks and spheres resulting of radiation pressure (KING, 1934), and some successful experiment of acoustic levitation where presented between 1930 and 1970 (BUCKS; MULLER, 1933) (CLAIR, 1949) (HANSON; DOMICH; ADAMS, 1964). However, it was not until the 1970s that powerful acoustic drivers became easy enough to produce to allow practical application of acoustic levitators. In the 1980s, most of the study of acoustic levitators were made by the NASA, who conducted research on microgravity and containerless material processing (WANG et al., 1986). In the early 2000s, Xie et al. published several studies on the effect of the geometry, the temperature, size and shape of the particle levitated on the axisymmetric (also called single-axis) acoustic levitator (XIE; WEI, 2001, 2002; XIE et al., 2002; XIE; WEI, 2003; XIE et al., 2006; XIE; WEI, 2007), which is now the most used and studied type of acoustic levitator. In this device, particles are entrapped at the pressure nodes of the standing wave established between a radiating plate and a reflector. Until 2010, acoustic levitation devices were limited to almost static experiments, with the maximum displacement possible of only a few millimeters (KOZUKA; TUZIUTI, 1998). The first acoustic levitation transportation systems are presented by D. Koyama and K. Nakamura, allowing the displacement of particles in a linear trajectory (KOYAMA; NAKAMURA, 2010b) and in a circular trajectory (KOYAMA; NAKAMURA, 2010a).

\section{$1.2 \quad$ Acoustic levitation phenomenon}

The acoustic levitation phenomenon uses standing acoustic wave to trap particles and make them levitate. This phenomenon can be achieved due to interference of two traveling waves with same frequency and amplitude traveling in opposite directions, creating high pressure regions (anti-nodes) and low pressure regions (nodes). The nodes and anti-nodes 
allow the trapping, and thus the suspension, of particles in a fluid (usually, but not only, air). In a typical levitator, described in fig. 1.2, the acoustic standing wave is generated by a piezoelectric ultrasonic transducer and a reflector separated by an integral number of half wavelengths. The forces on an object subjected to an acoustic field result from the nonlinear relationship between the pressure and velocity in an acoustic medium.

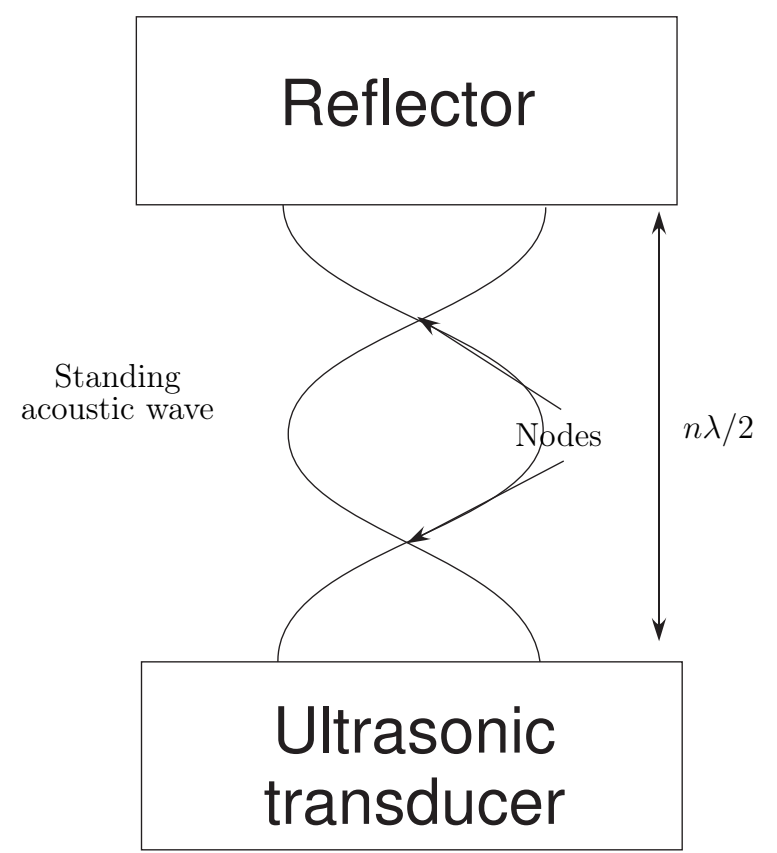

Figure 1.2: Principle of acoustic levitation

\subsection{Single-axis acoustic levitator}

The single-axis (or axisymmetric) acoustic levitator is the simplest and most common acoustic levitator, and consists of an ultrasonic transducer and a reflector, arranged typically with their axis in the gravitational direction. Most of the single-axis acoustic levitator uses a Langevin transducer, which consists of pairs of piezoelectric rings clamped between two loading masses, coupled with a mechanical amplifier (horn), as shown in fig. 1.3. A lot of studies have been made to optimize the geometry of the transducer and the reflector (XIE; WEI, 2001; ANDRADE, 2010) in order to levitate heavier object and to have a more stable levitation. In addition, the position and the force applied on a particle can be determined by the finite element method (ANDRADE, 2010), by the boundary element method (XIE; WEI, 2002) or using Rayleigh integral accounting multiple reflection of the acoustic wave between the transducer and the reflector (ANDRADE; ADAMOWSKI, 2011).

Single-axis acoustic levitators are mostly used when a particle needs to be standing still in the air, and was used, for example, to determine the resonant modes of a liquid droplet in microgravity (APFEL et al., 1997). 


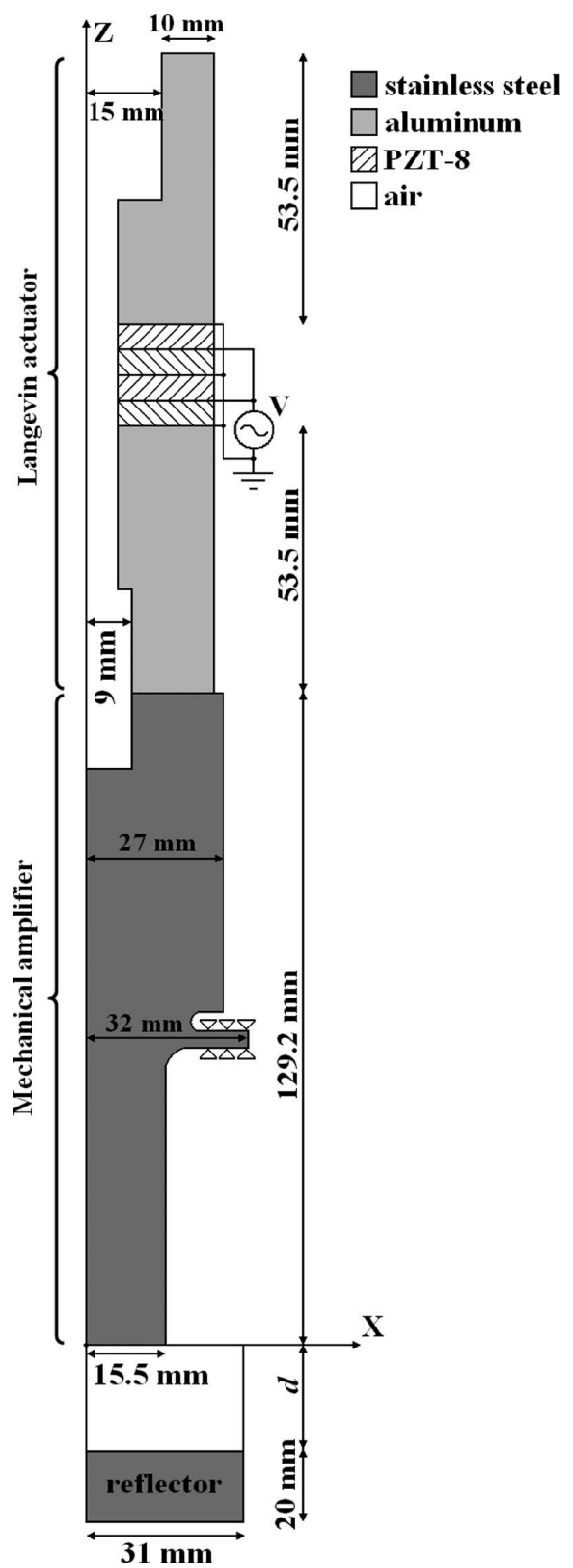

Figure 1.3: Axisymmetric model of a single-axis acoustic levitator from (ANDRADE, 2010).

\subsection{Acoustic levitation transportation systems}

The transportation and manipulation of particles using acoustic levitation in a $2 \mathrm{D}$ plane can be very useful in the fields of micromachine, biotechnology and powder processing. The first successful experiment was made by (KOZUKA; TUZIUTI, 1998), where a particle is displaced with precision over a few millimeters using two transducers whose sound beam axes are crossing each other.

However the first long distance acoustic levitation transportation systems was developed by D. Koyama and K. Nakamura, following previous works in near field acoustic levitation (KOYAMA; NAKAMURA; UEHA, 2007). In the case of an acoustic levitation linear transportation system, a plate is excited at each end by Langevin transducers in order 
to obtain flexural vibrations along the plate, and a reflector is installed parallel to the vibrating plate at a distance equals to integral number of half wavelengths of standing wave in air, as show in fig. 1.4. The prototype plate dimensions are $605 \times 60 \times 3 \mathrm{~mm}$ and the $33^{\text {rd }}$ flexural mode is used at the frequency of $22.5 \mathrm{kHz}$. The pressure nodes, and thus the levitated particles, are moved by changing the driving phase difference between the two transducers (KOYAMA; NAKAMURA, 2010b). The maximum distance achieved by a particle with this system is $60 \mathrm{~mm}$ with an accuracy of $0.046 \mathrm{~mm} / \mathrm{deg}$.

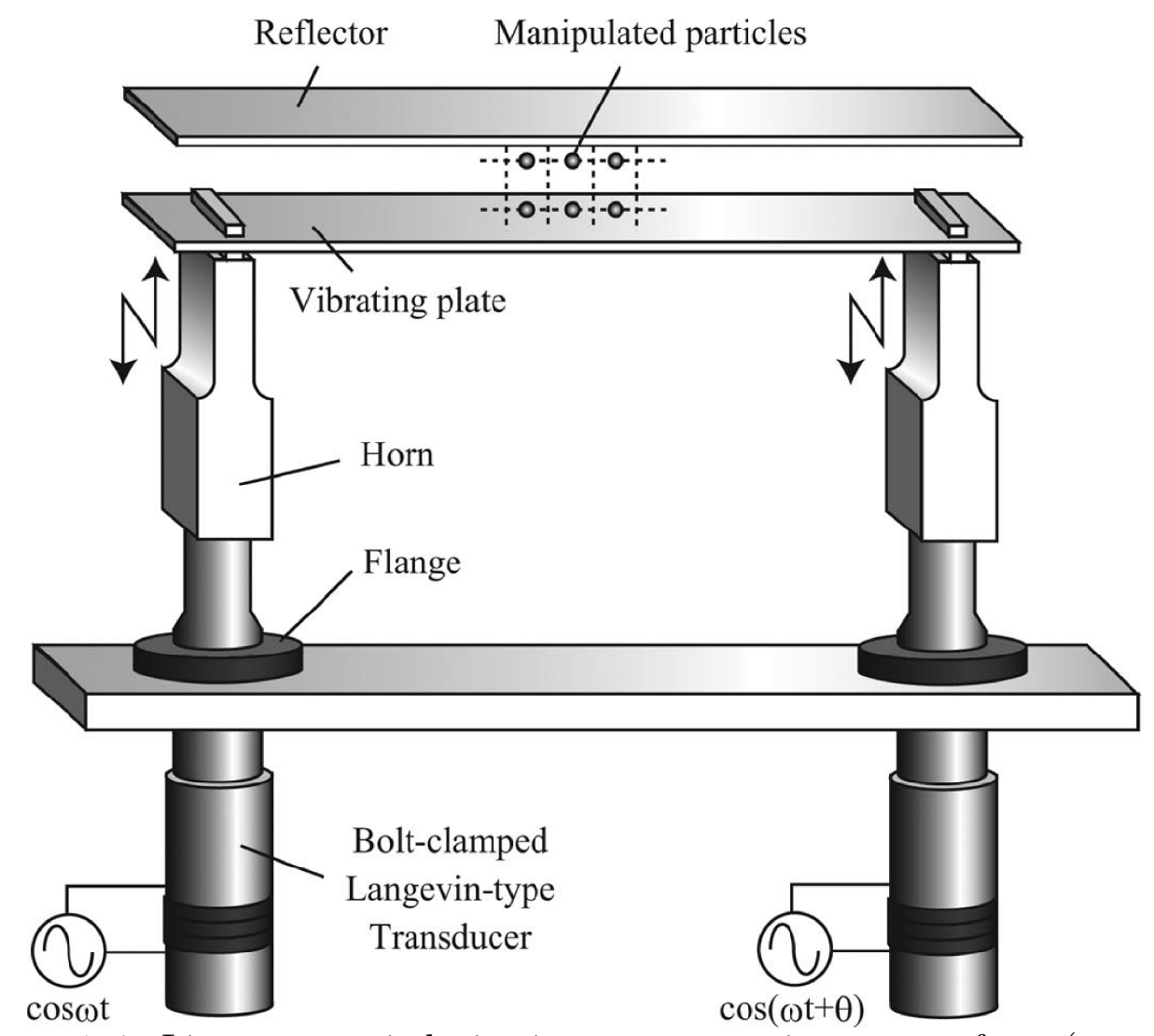

Figure 1.4: Linear acoustic levitation transportation system from (KOYAMA; NAKAMURA, 2010b).

D. Koyama and K. Nakamura also worked on an acoustic levitation circular transportation system (KOYAMA; NAKAMURA, 2010a). Their prototype is similar to an ultrasonic rotative motor, using an aluminium disc coupled with a piezoelectric ring divided into 24 equals elements, as shown in fig. 1.5. The aluminium disc diameter was $30 \mathrm{~mm}$ with a thickness of $0.5 \mathrm{~mm}$ and the mode used was the $(1,4)$ mode (note: (modal circle, modal line)) at the frequency of $46.6 \mathrm{kHz}$. The particle is manipulated by switching between a 3-3 drive and a 4-2 drive: since there are 24 piezoelectric elements and the fourth flexural mode is excited, six elements are used to generate a wavelength in the disc. The 3-3 drive means three electrodes have a phase difference of $\pi$ with the other three while the 4-2 drive means four electrodes have a phase difference of $\pi$ with the other two. The authors successfully moved polystyrene particles in a circular trajectory, with step of $7.5 \mathrm{deg}$ between each position and a maximum speed of $97 \mathrm{~mm} / \mathrm{s}$. 


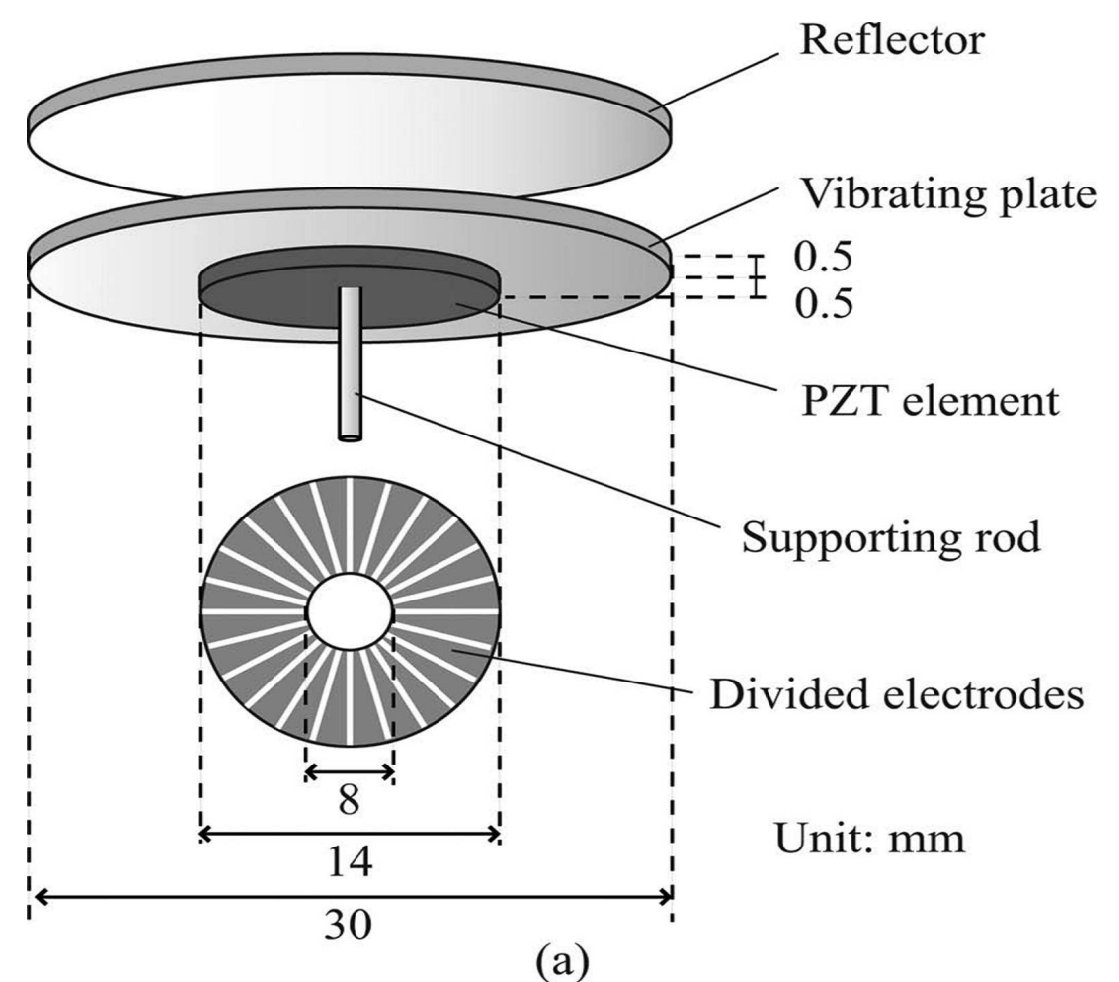

Figure 1.5: Circular acoustic levitation transportation system from (KOYAMA; NAKAMURA, 2010a).

The idea of D. Koyama and K. Nakamura is to couple both acoustic levitation transportation system, linear and circular, in order to be able to integrate acoustic levitation transportation system in the industry. Unfortunately, as reported in their paper for the linear transportation system, the particle cannot be moved behind the transducers and thus, making this idea impossible with their prototypes.

Another type of long distance acoustic levitation transportation is found by D. Foresti et al., where the particles are moved from a single-axis levitator to another by decreasing the pressure amplitude of the sender and increasing the pressure amplitude of the receiver. This method allow a wide palette of application experiments, such as contactless droplet coalescence and mixing, solid-liquid encapsulation, absorption, dissolution, and DNA transfection (FORESTI; NABAVI, 2013).

\subsection{Other applications of the acoustic levitation}

Other works have been done in order to allow particle manipulation using acoustic levitation. S. A. Seah et al. used a circular array of 8 transducers to manipulate the acoustic pressure field and thereby translated particle in a $2 \mathrm{D}$ vertical plane with an accuracy of $0.09 \mathrm{~mm}$ (SEAH et al., 2014). In a similar manner, Y. Ochiai et al. used four ultrasonic phased array as transducers and placed them facing each other, allowing three-dimensional manipulation of particle in air (OCHIAI; HOSHI; REKIMOTO, 2014). By 
extension, various works on acoustofluidics use the acoustophoresis phenomenon in liquid to manipulate cells (LENSHOF; MAGNUSSON; LAURELL, 2012).

\subsection{Motivation of study}

Because of its high potential of application in the micromachining, chemical and pharmaceutical industries, the acoustic levitation phenomenon and its applications have been the focus of a lot of research in the last two decades. Until 2010, these applications were limited to almost static levitation, however now, the theory behind the contactless transportation of particle have been developed, we can imagine lots of different applications in the industry, mainly pharmaceutical which needs to deal with high purity or hazardous materials. For example, (FORESTI; NABAVI, 2013) use acoustic levitation to transport droplets or particles and mix them without any contact, which is critical for pharmaceutical and chemical industry (see fig. 1.6).

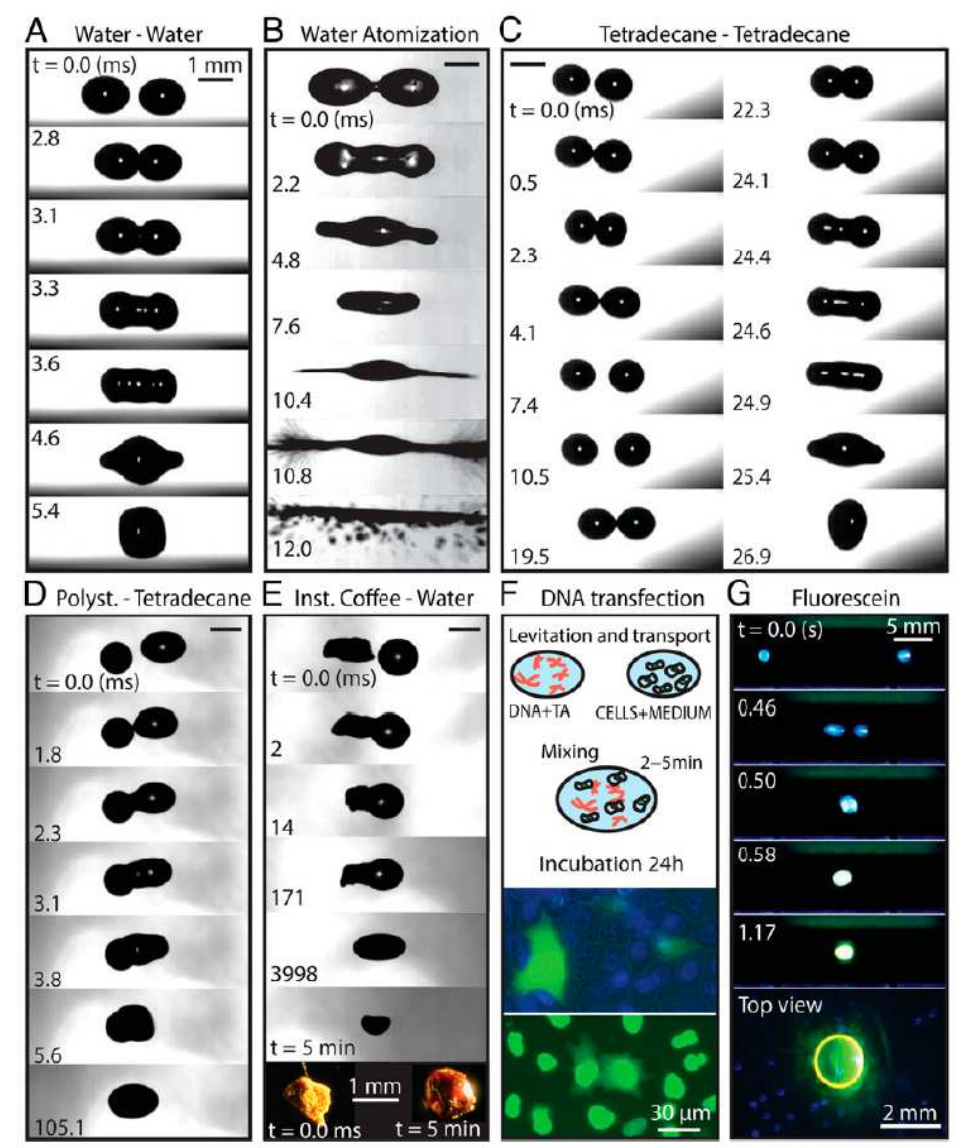

Figure 1.6: Contactless mixing of droplets and particles (FORESTI; NABAVI, 2013).

Since the first prototype was made in 2010 , there may be a lot of way to improve acoustic levitation linear transportation systems and in this work a different prototype is proposed. 


\subsection{Objectives}

This project looks into the principles and concepts behind acoustic levitation linear transportation of particles. The scope includes the following areas:

- Study on the principle of acoustic levitation transportation.

- Complete finite element modeling of an acoustic levitation linear transportation system.

- Design and fabrication of prototype.

- Evaluation of proposed theoretical model.

- Testing and evaluation of prototype.

- Verification of theoretical model with experimental results.

\subsection{Dissertation overview}

The second chapter focus on the theory behind acoustic levitation, i.e. the theory of acoustic wave, the forces resulting of an acoustic field and the transducer used in acoustic levitation. The third chapter presents the theoretical modeling of a ring-type vibrator, and chapter four the numerical modeling of the acoustic levitation transportation system designed by (KOYAMA; NAKAMURA, 2010a), and also the modeling of a new kind of vibrator. A prototype with this new kind of vibrator is then designed by using iterative design in the fifth chapter. This prototype is then analysed numerically and compared to the prototype of (KOYAMA; NAKAMURA, 2010a), before being manufactured and experimentally evaluated. The last chapter conclude on the efficiency of the proposed system and open the way to future work. 


\section{Theory of acoustic levitation}

\section{$2.1 \quad$ Acoustic plane waves}

Acoustic waves in non-viscous fluid are longitudinal waves, meaning that the particles transmitting the wave move back and forth in the direction of propagation of the wave, producing alternate regions of compression and rarefaction. The theory behind acoustic plane wave can be linear, in the case of waves with a small amplitude, or non-linear if the amplitudes involved are high. In the particular case of acoustic levitation, the acoustic forces exerted on a solid are the result of non-linear acoustic theory, however, the pressure field can be described by using the linear theory. Even without the presence of a wave the molecules in a fluid are in constant motion, however from a statistical point of view a small volume element can be treated as an unchanging unit, since the molecules leaving its confine are replaced by a equal number possessing identical properties. That is why the terms particle displacement and particle velocity are used when speaking about acoustic wave.

When the pressure variation, and so the amplitude of the acoustic plane wave, is small, the linear acoustic theory can be used. In the case of a non-viscous fluid, the governing equation is (KINSLER et al., 1982):

$$
\frac{\partial^{2} p}{\partial t^{2}}=c^{2} \nabla^{2} p
$$

where $p$ is the instantaneous pressure at any point, $t$ is the time and $c$ is the velocity of propagation of the wave. A relationship between the particle velocity vector $\mathbf{u}$ and the pressure gradient can be found in the linear Euler's equation:

$$
\rho_{0} \frac{\partial \mathbf{u}}{\partial t}=-\nabla p
$$

where $\rho_{0}$ is the constant equilibrium density of medium. 


\subsection{Acoustic radiation force: Gor'kov theory}

Acoustic radiation force results from the nonlinear effect of an acoustic wave. In 1962, Gor'kov (GOR'KOV, 1962) proposed a formulation for the acoustic radiation force based on the energy stored in a one-dimensional plane standing wave. The potential $U$ of the acoustic force $F$ that acts on small spherical particle in an arbitrary acoustic field in an non-viscous ideal fluid is:

$$
U=2 \pi \alpha^{3}\left(\frac{\overline{p_{i n}^{2}}}{3 \rho_{f} c^{2}} f_{1}-\frac{\rho_{f} \overline{u_{i n}^{2}}}{2} f_{2}\right)
$$

where $\alpha$ is the radius of the levitated sphere, $\rho_{f}$ the density of the fluid, $\overline{p_{i n}^{2}}$ and $\overline{u_{i n}^{2}}$ are the mean-square fluctuations of the pressure and velocity of the wave at the point where the particle is located. In the case of a rigid sphere $f_{1}=f_{2}=1$. Finally, the acoustic force components are obtained by:

$$
F=-\nabla U
$$

In 1985, Barmatz and Collas (BARMATz, 1985) applied the method of Gor'kov to determine the acoustic radiation potential on a sphere for standing wave modes in rectangular, cylindrical and spherical geometries, in the case where the radius of the suspended sphere is very small compared to the acoustic wavelength $\left(\alpha<<\lambda_{a}\right)$. For comparison and computational purpose, they defined the dimensionless potential as:

$$
\tilde{U}=\frac{U}{\pi \alpha^{3} \rho_{f} u_{0}}
$$

where $u_{0}$ is the particle maximum velocity. Since the forces exerted on a small sphere are equal to the negative gradient of the levitation potential, as expressed in 2.4 , a small sphere can be levitated at a levitation potential well. An example plot of the dimensionless potential $\tilde{U}$ and the resulting levitating particle is shown fig.2.1, from the work of (ANDRADE, 2010).

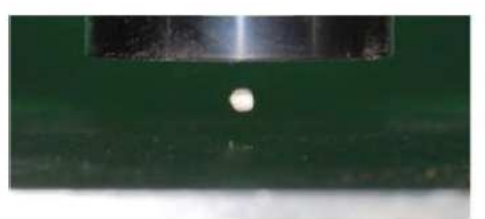

(a)

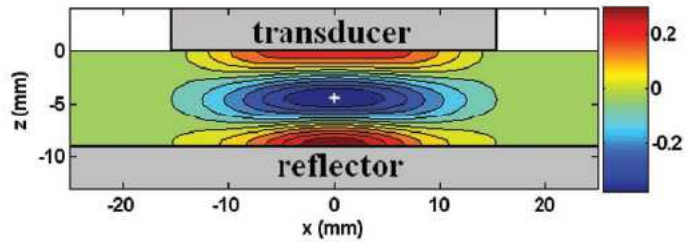

(b)

Figure 2.1: a/ Levitating particle; b/ dimensionless levitation potential (ANDRADE, 2010). 


\subsection{Acoustic transducers}

In recent papers on acoustic levitation, most of the acoustic transducers are piezoelectric ultrasonic transducers. It is usually composed of a Langevin transducer, which transform the electrical energy inside the piezoelectric ceramics into mechanical energy, a mechanical amplifier, that amplifies the output displacement, and sometimes a horn is added as a second mechanical amplifier in order to obtain very high displacement output. Those parts all function as half-wavelength resonators, meaning that the length of the part is equal to half the wavelength of the first longitudinal mode.

\subsubsection{Piezoelectricity}

The piezoelectric phenomenon can be observed in some non conductive materials, as some crystals are able to polarize themselves under mechanical constraints, and it is defined as the conversion of mechanical energy into electrical energy (called the direct effect of piezoelectricity) and reciprocally (the indirect effect). The piezoelectric effect is presented by 2 coupled equations that bind the electrical variables (the electric charge displacement $\mathbf{D}$ or the electrical field $\mathbf{E}$ ) and the mechanical variables (the strain $\mathbf{S}$ or the stress $\mathbf{T}$ ). The strain-charge form is presented here in 2.6.

$$
\left\{\begin{array}{l}
\mathbf{S}=\mathbf{S}_{\mathbf{E}} \cdot \mathbf{T}+\mathbf{d}^{t} \cdot \mathbf{E} \\
\mathbf{D}=\mathbf{d} \cdot \mathbf{T}+\varepsilon_{\boldsymbol{T}} \cdot \mathbf{E}
\end{array}\right.
$$

Table 2.1: Matrix variables used in the piezoelectric constitutive equations

\begin{tabular}{cccc}
\hline Symbol & Meaning & Type & Size \\
\hline $\mathbf{D}$ & Electric charge density in $\frac{C}{m^{2}}$ & Electric & $(3,1)$ \\
$\mathbf{E}$ & Electric field in $\frac{N}{C}$ & & $(3,1)$ \\
$\boldsymbol{\varepsilon}_{\boldsymbol{T}}$ & Electric permittivity in $\frac{F}{m}$ & & $(3,3)$ \\
\hline $\mathbf{S}$ & Strain in $\frac{m}{m}$ & Mechanical & $(6,1)$ \\
$\mathbf{T}$ & Stress in $\frac{N}{m^{2}}$ & & $(6,1)$ \\
$\mathbf{S}_{\mathbf{E}}$ & Compliance in $\frac{m^{2}}{N}$ & & $(6,6)$ \\
\hline \multirow{2}{*}{$\mathbf{d}$} & Piezoelectric coupling coefficients & Piezoelectric & $(3,6)$ \\
& for strain-charge form in $\frac{C}{N}$ & & \\
\hline
\end{tabular}




\subsubsection{Langevin transducer coupled with mechanical amplifier}

A Langevin transducer works as a half-wavelength resonator, and usually have a very small bandwidth, meaning that they can convert efficiently the electrical energy inside the piezoelectric ceramics into mechanical displacement only for a short range of frequencies. The Langevin transducer consists of a stack of piezoelectric ceramics, polarized longitudinally, and embedded between two metal cylinders, as shown in fig.2.2.

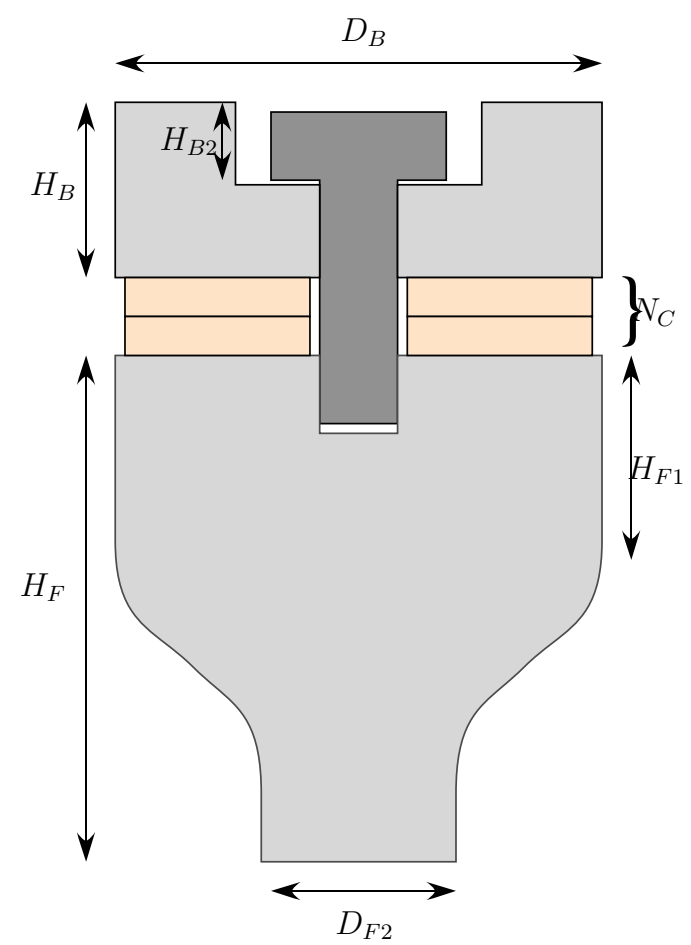

Figure 2.2: Parameterized schematic of a Langevin transducer coupled with a mechanical amplifer.

A very complete design guide of a bolt-camped Langevin transducer coupled with mechanical amplifier was made by (ABDULLAH; SHAHINI; PAK, 2009). This article explain in a very detailed way how to correctly choose the parameters of the transducer presented fig.2.2, its materials, the prestress exerted by the bolt on the piezoelectric ceramics and its assembly.

\subsubsection{Acoustical horn}

In order to amplify even more the vibration amplitude output of the transducer, an acoustical horn can be added. Acoustic horns are common in the ultrasonic machining industry. Usually, there is four kind of profile for acoustical horn: cylindrical, stepped, conical and exponential, shown in fig.2.3.

As for the Langevin transducers, the acoustical horn works as a half-wavelength resonator, have a very small bandwidth, and the displacement amplification mostly 


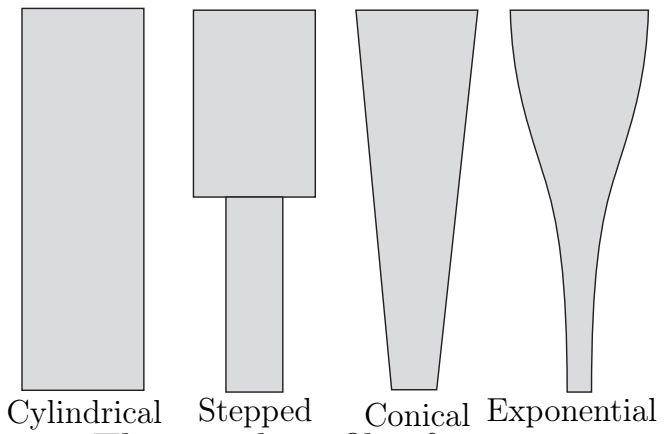

Figure 2.3: The usual profiles for an acoustical horn.

depend of the ratio between the area of the upper end and lower end. The finite elements design process is fully explained by (AMIN; AHMED; YOUSSEF, 1995), and this article will be used to design the horns of this project. 


\section{Theoretical modeling of the ring-type vibrator}

In the acoustic levitation transportation system described in (KOYAMA; NAKAMURA, 2010b), the standing wave of the bending vibrator generates an acoustic standing wave between the vibrator and the reflector where small particles can levitate at pressure nodes. By changing the spatial phase of the standing wave in the vibrator, the particle levitating is moved by the same distance as the vibrator standing wave. In the original paper, the distance $\Delta x$ traveled by the standing wave of the vibrator is the result of a temporal phase shift $\Delta \varphi$ between the two Langevin transducers. This type of traveling wave presents a non linear relation between $\Delta x$ and $\Delta \varphi$, and the amplitude of the standing wave have a $30 \%$ variation (KOYAMA; NAKAMURA, 2010b) (maximum for $\Delta \varphi=0$ and minimum for $\Delta \varphi=\pi)$. Another inconvenient of this system is that the particle cannot be transported to the extremities of the plate, thus making difficult the transfer of the particle from one transportation system to another. On the last point, the original author and his coworkers recently published a paper where this problem is solved by ejecting the particle between the two systems (KASHIMA; MURAKAMI; KOYAMA, 2014). The objective of this section is to model the ring-type vibrator in order to evaluate its use in an acoustic levitation linear transportation system.

\subsection{Working principle}

A ring-type structure consists of two straight beams of length $L$ and two curved beams of radius $R$. It was first developed by Seemann (SEEMANN, 1996) as a new type of linear ultrasonic traveling wave motor. Beam theory shows that, for certain ratios of $L / R$, double eigenfrequencies exist, meaning that it is possible to generate a traveling wave by superposing two standing waves with a $\pi / 2$ phase difference in both time and space, in the same way as a traveling wave rotary motor. Figure 3.1, from the original paper, shows the ring-type structure and the frequency difference between the two orthogonal resonant modes. 

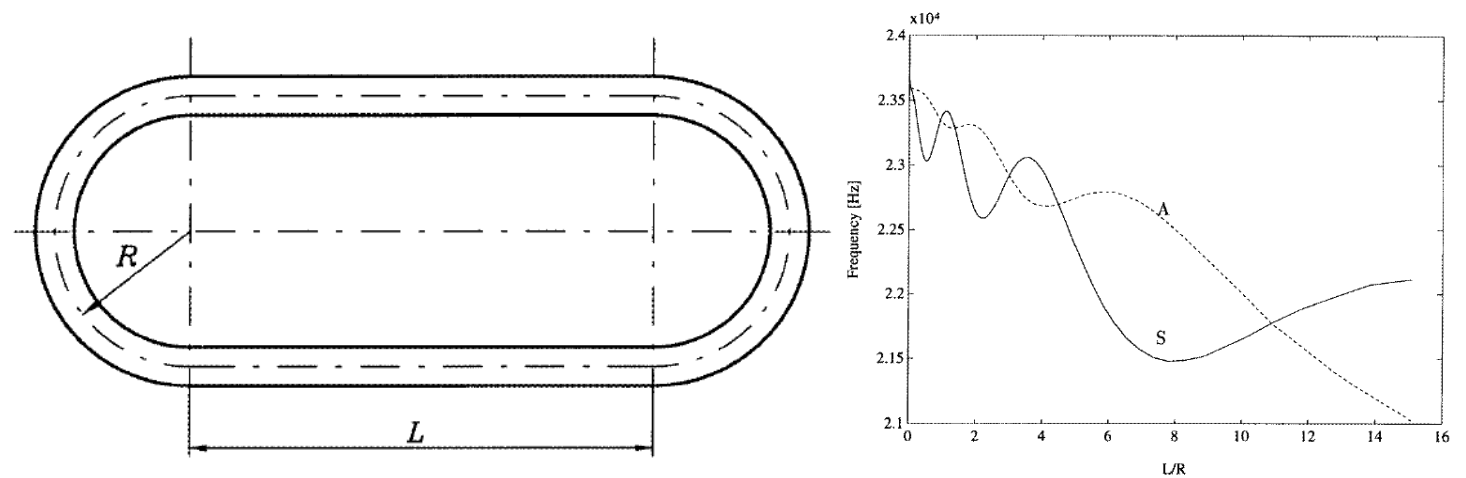

Figure 3.1: Left: ring-type vibrator, right: frequency difference between the two orthogonal modes as presented by (SEEMANN, 1996).

In the ultrasonic linear motor presented by (SEEMANN, 1996), the excitation is realized by using piezoceramics of the length of $\lambda_{v} / 2$ directly bonded to the structure, with their polarity alternated, as shown in fig. 3.2. Finally, the experimental setup used in this case is shown in fig. 3.3 .

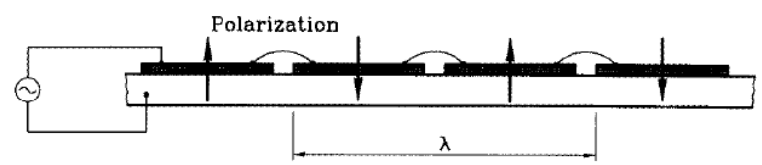

Figure 3.2: Polarization of the piezoceramics on the stator (SEEMANN, 1996).

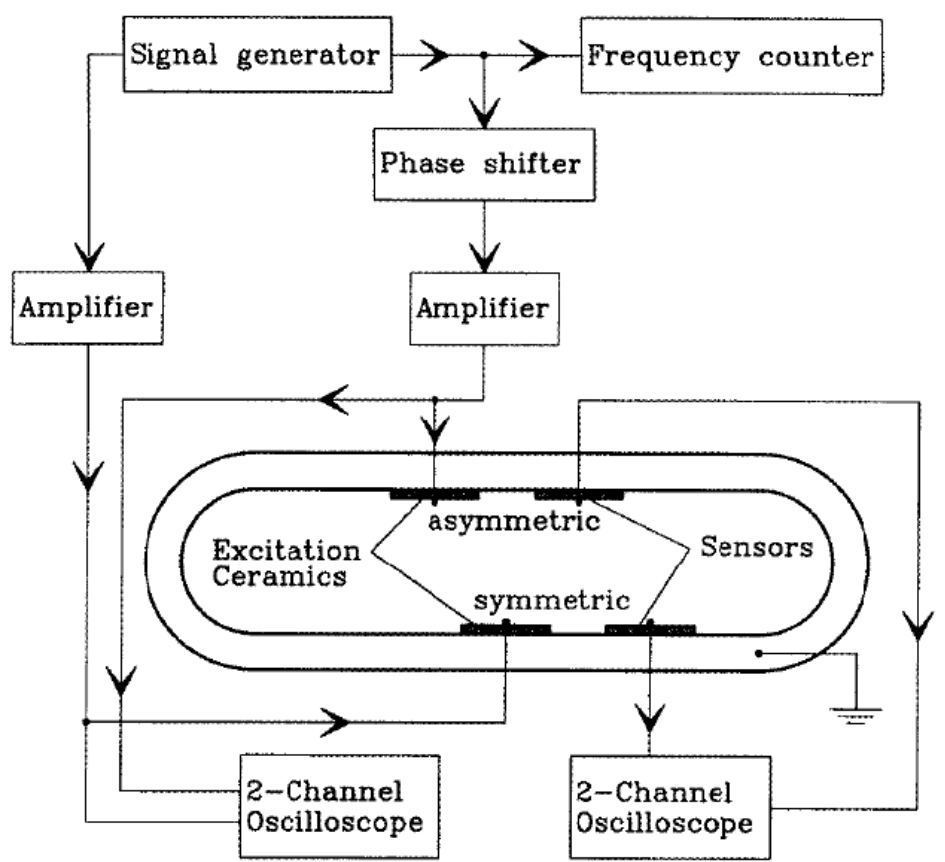

Figure 3.3: Experimental setup of the ultrasonic motor (SEEMANN, 1996).

In the conclusion of (SEEMANN, 1996), the author comments that, due to the uncertainties in the production process and the unprecise ceramic locations, the 
frequencies of the two modes did not coincide and manipulation on the stator had to be made in order to correct it.

This ring-type structure presents the two advantage desired to increment the work of (KOYAMA; NAKAMURA, 2010a): the possibility to move the particle everywhere around the vibrator and, by using another type of excitation, a linear relation between the distance traveled by the wave and the input variable. Here, instead of bonding piezoceramics to the vibrator, two high-power acoustic transducers will be used in order to produce the high displacement required for acoustic levitation and also to avoid the problem encountered by Seemann where the two orthogonal mode frequencies were off due to imprecision in the ceramic locations. The vibrator model used in this project is presented in fig. 3.4.

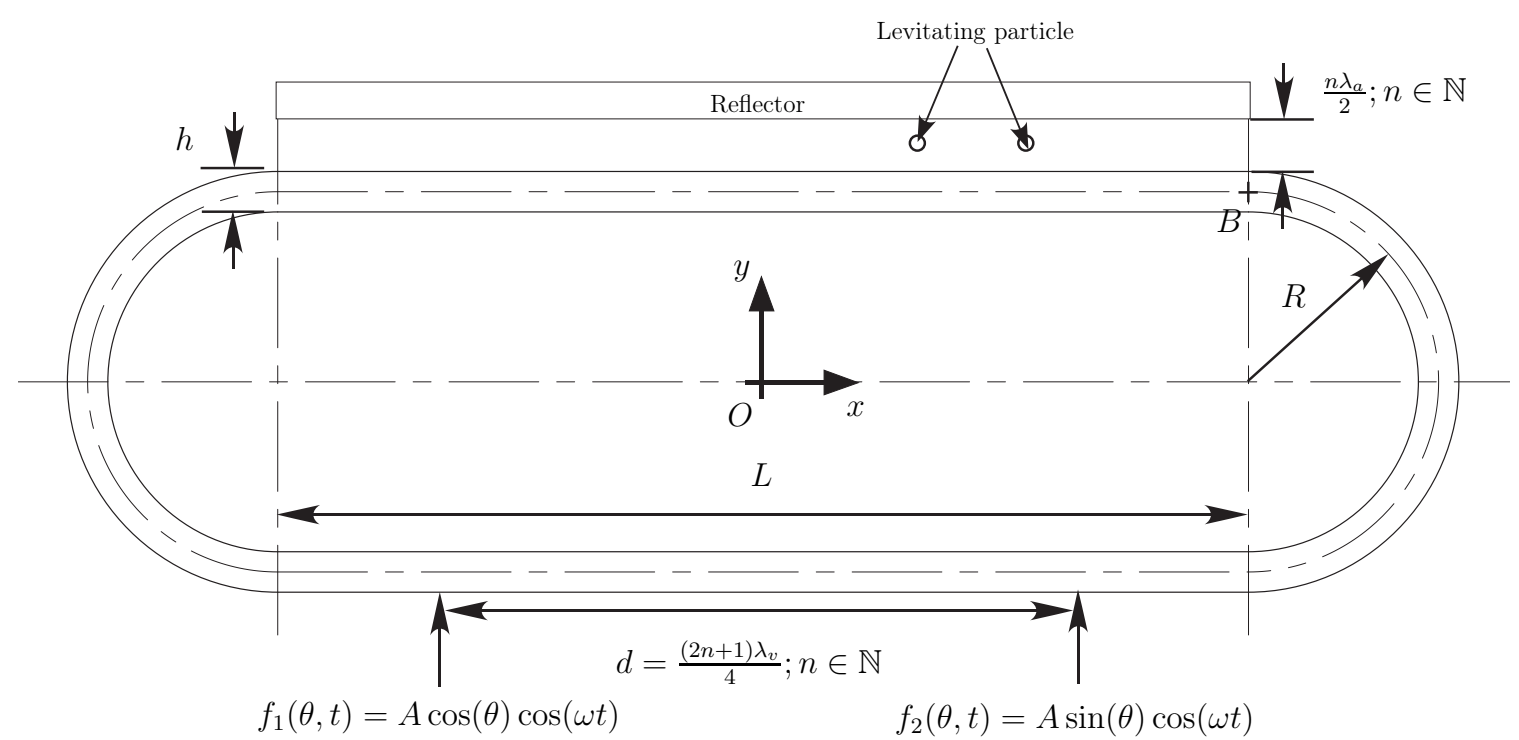

Figure 3.4: Ring-type vibrator.

The excitation is set as $f_{1}(\theta, t)=A \cos (\theta) \cos (\omega t)$ and $f_{2}(\theta, t)=A \sin (\theta) \cos (\omega t)$, where $A$ is the excitation amplitude, $\theta$ a variable, and $\omega$ the angular frequency. The length $L$ and radius $R$ are set to allow two pseudo orthogonal mode at the frequency $\omega$, and the distance $d$ between the two excitation points as $d=\frac{(2 n+1) \lambda_{v}}{4} ; n \in \mathbb{N}$, where $\lambda_{v}$ is the wavelength of the standing wave inside the vibrator, thus giving a spatial phase shift of $\pm \pi / 2$ between the two standing wave generated by $f_{1}$ and $f_{2}$. The resulting flexural wave of the neutral axis in the straight parts of the vibrator is:

$$
\begin{aligned}
u & =A \cos (\theta) \cos (k x) \cos (\omega t) \pm A \sin (\theta) \sin (k x) \cos (\omega t) \\
& =A \cos (k x \mp \theta) \cos (\omega t)
\end{aligned}
$$

where $k=\frac{2 \pi}{\lambda_{v}}$ is the wave number. Equation 3.1 shows that $\theta$ is the spatial phase of the standing wave in the vibrator, meaning that by changing the value of $\theta$ the standing wave will move by $\Delta x=\frac{\theta}{k}$, and therefore moving the levitating particle with it. 


\subsection{Analytical modeling of a ring-type structure}

A modeling of a ring-type vibrator for ultrasonic traveling wave motor was proposed by (LIU et al., 2013) using Euler-Bernoulli beam theory, however since the frequency of the prototype is expected to be higher than $20 \mathrm{kHz}$, the ring-type vibrator is modeled here using Timoshenko beam theory for both straight and curved part. The objective here is to develop an analytical method that will serve as a paradigm for finding the right $\frac{R}{L}$ ratio that gives two pseudo orthogonal modes at the same frequency.

\subsubsection{Straight part}

The Timoshenko beam theory takes into account shear deformation and rotational effects, which makes it suitable for describing the behavior of beams subjected to high-frequency excitation. The displacement of the Timoshenko beam represented at fig. 3.5 are:

$$
u_{x}(x, y, z, t)=u_{s}-y \varphi_{s}(x, t) ; u_{y}(x, y, z, t)=v_{s}(x, t) ; u_{z}(x, y, z, t)=0
$$

where $u_{x}, u_{y}, u_{z}$ are the components of the displacement vector, $u_{s}$ is the horizontal motion of the beam, $v_{s}$ the vertical motion of the midline of the beam and $\varphi_{s}$ the rotation of the normal of the midline.

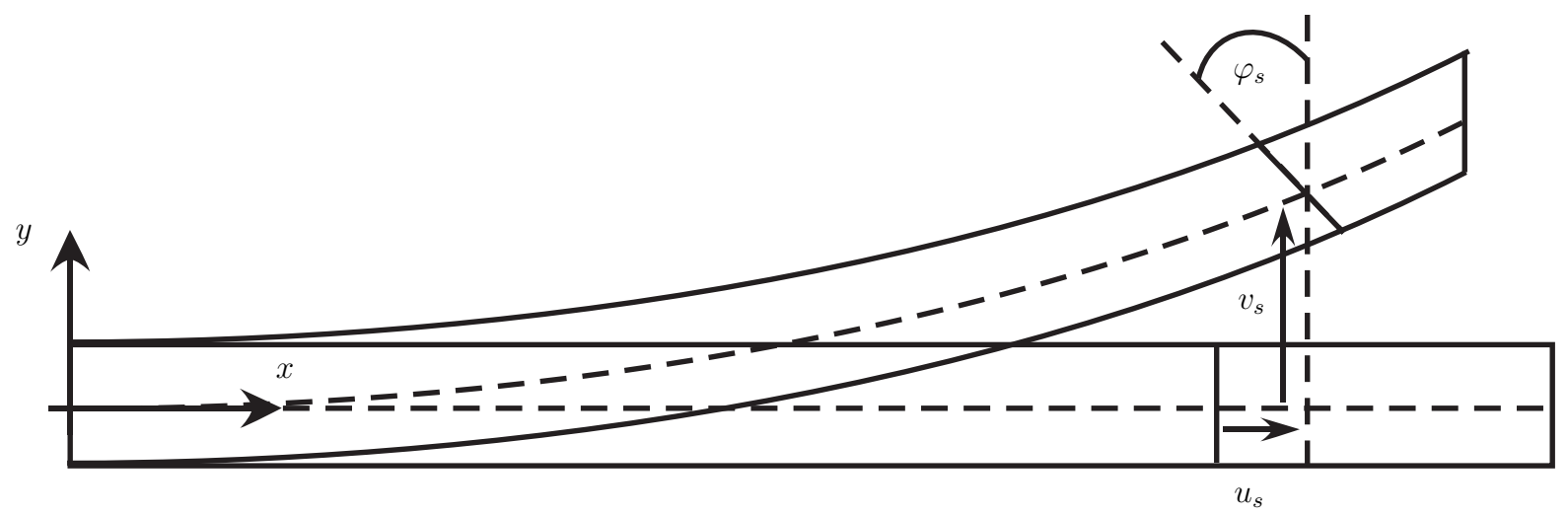

Figure 3.5: Deformation of a Timoshenko beam.

The resulting forces inside the beam are:

$$
\begin{gathered}
N_{s}=E A\left(\frac{\partial u_{s}}{\partial x}\right) \\
M_{s_{x x}}=-E I\left(\frac{\partial \varphi_{s}}{\partial x}\right)
\end{gathered}
$$




$$
Q_{s_{x}}=k G A\left(\frac{\partial v_{s}}{\partial x}-\varphi_{s}\right)
$$

where $N_{s}$ is the normal force, $M_{s_{x x}}$ the bending moment, $Q_{s_{x}}$ the shear force, $E$ the Young's modulus, $k$ the Timoshenko shear correction coefficient, $G$ the shear modulus, $I$ the second moment of area, $A$ the beam section area and $\rho$ the density.

The kinetic energy $\mathcal{T}_{s}$ and the deformation energy $\mathcal{V}_{s}$ of the straight part of the beam are:

$$
\begin{gathered}
\mathcal{T}_{s}=\frac{1}{2} \int_{0}^{L}\left[\rho A\left(\frac{\partial u_{s}}{\partial t}\right)^{2}+\rho A\left(\frac{\partial v_{s}}{\partial t}\right)^{2}+\rho I\left(\frac{\partial \varphi_{s}}{\partial t}\right)^{2}\right] \mathrm{d} x \\
\mathcal{V}_{s}=\frac{1}{2} \int_{0}^{L}\left[E A\left(\frac{\partial u_{s}}{\partial x}\right)^{2}+k G A\left(\frac{\partial v_{s}}{\partial x}-\varphi_{s}\right)^{2}+E I\left(\frac{\partial \varphi_{s}}{\partial x}\right)^{2}\right] \mathrm{d} x
\end{gathered}
$$

The Euler-Lagrange equation of the problem is:

$$
\mathcal{L}_{s}=\mathcal{T}_{s}-\mathcal{V}_{s}
$$

Applying the Hamilton's principle $\delta \int_{t_{1}}^{t_{2}} \mathcal{L}_{s} \mathrm{~d} t=0$ (detailed in appendix B.1) yield:

$$
\begin{gathered}
\rho A \frac{\partial^{2} u_{s}}{\partial t^{2}}-E A \frac{\partial^{2} u_{s}}{\partial x^{2}}=0 \\
\rho A \frac{\partial^{2} v_{s}}{\partial t^{2}}-k G A\left(\frac{\partial^{2} v_{s}}{\partial x^{2}}-\frac{\partial \varphi}{\partial x}\right)=0 \\
\rho I \frac{\partial^{2} \varphi_{s}}{\partial t^{2}}-E I \frac{\partial^{2} \varphi_{s}}{\partial t^{2}}-k G A\left(\frac{\partial v_{s}}{\partial x}-\varphi_{s}\right)=0
\end{gathered}
$$

\subsubsection{Curved part}

The curved part of the ring-type structure is also modeled considering rotational inertia and shear deformation as in the Timoshenko straight beam theory. The curved beam is represented fig.3.6.

where $u_{c}$ the axial displacement, $v_{c}$ the radial displacement, $\varphi_{c}$ the shear angle and $R$ the curvature radius.

The axial force $N_{c}$, the shear force $Q_{c_{s}}$ and the bending moment $M_{c_{s s}}$ are expressed as follows: 


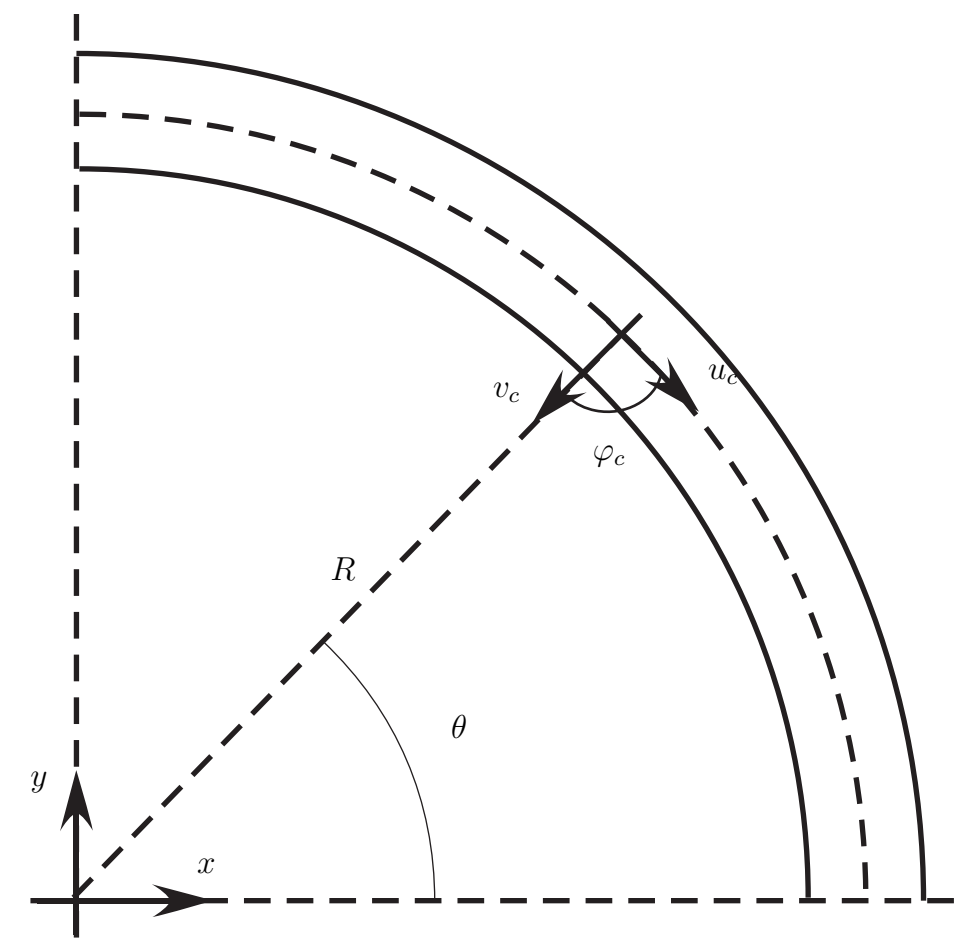

Figure 3.6: Deformation of a curved Timoshenko beam.

$$
\begin{gathered}
N_{c}=E A\left(\frac{\partial v_{c}}{\partial s}+\frac{u_{c}}{R}\right) \\
Q_{c_{s}}=k A G\left(\frac{\partial v_{c}}{\partial s}+\frac{u_{c}}{R}-\varphi_{c}\right) \\
M_{c_{s s}}=E I\left(\frac{\partial \varphi_{c}}{\partial s}\right)
\end{gathered}
$$

where $s$ is the arc length from the midline of the curved beam, and $\mathrm{d} s=R \mathrm{~d} \theta$.

In the case of closed ring, the hypothesis of inextensibility of the midline can be made (CHIDAMPARAM; LEISSA, 1993), and thus $N_{c}=0$, which gives the following relation between $u_{c}$ and $v_{c}$ :

$$
v_{c}=\frac{\partial u_{c}}{\partial \theta} ; u_{c}=-\frac{\partial v_{c}}{\partial \theta}
$$

The kinetic energy $\mathcal{T}_{c}$ and the deformation energy $\mathcal{V}_{c}$ of the curved part of the beam are:

$$
\mathcal{T}_{c}=\frac{1}{2} \int_{-\frac{\pi}{2}}^{\frac{\pi}{2}}\left[\rho A R\left(\frac{\partial u_{c}}{\partial t}\right)^{2}+\rho A R\left(\frac{\partial v_{c}}{\partial t}\right)^{2}+\rho I R\left(\frac{\partial \varphi_{c}}{\partial t}\right)^{2}\right] \mathrm{d} \theta
$$




$$
\mathcal{V}_{c}=\frac{1}{2} \int_{-\frac{\pi}{2}}^{\frac{\pi}{2}}\left[\frac{k G A}{R}\left(\frac{\partial v_{c}}{\partial \theta}+u_{c}-R \varphi\right)^{2}+\frac{E I}{R}\left(\frac{\partial \varphi_{c}}{\partial \theta}\right)^{2}\right] \mathrm{d} \theta
$$

The Euler-Lagrange equation of the problem is:

$$
\mathcal{L}_{c}=\mathcal{T}_{c}-\mathcal{V}_{c}
$$

Applying the Hamilton's principle $\delta \int_{t_{1}}^{t_{2}} \mathcal{L}_{c} \mathrm{~d} t=0$ (detailed in appendix B.2) yield:

$$
\begin{gathered}
\frac{\rho R^{2}}{k G}\left[\frac{\partial^{2} u_{c}}{\partial t^{2}}-\frac{\partial^{2}}{\partial t^{2}} \frac{\partial^{2} u_{c}}{\partial \theta^{2}}\right]+\frac{\partial^{4} u_{c}}{\partial \theta^{4}}+2 \frac{\partial^{2} u_{c}}{\partial \theta^{2}}+u_{c}-R \varphi-R \frac{\partial^{2} \varphi}{\partial \theta^{2}}=0 \\
\frac{\rho I R}{k A G} \frac{\partial^{2} \varphi}{\partial t^{2}}-\frac{E I}{k A G R} \frac{\partial^{2} \varphi}{\partial \theta^{2}}+R \varphi-\frac{\partial^{2} u_{c}}{\partial \theta^{2}}-u_{c}=0
\end{gathered}
$$

\subsubsection{Boundary conditions}

Taking account of the symmetry with the center $(O, x, y)$ (see fig.3.4) of the structure, only two boundary conditions are needed to describe the structure vibration: same displacement and same slope of the middle line of the structure at the point $B$.

$$
\begin{aligned}
v_{s}\left(\frac{L}{2}, t\right) & =v_{c}\left(\frac{\pi}{2}, t\right) \\
\frac{\partial v_{s}}{\partial x}\left(\frac{L}{2}, t\right) & =\frac{\partial v_{c}}{\partial x}\left(\frac{\pi}{2}, t\right) \\
& =-\frac{\partial v_{c}}{R \partial \theta}\left(\frac{\pi}{2}, t\right)
\end{aligned}
$$

\subsubsection{Solutions}

Since only the harmonic solution of the flexural vibrations is of interest here, we can write:

$$
\left\{\begin{array}{l}
v_{s}(x, t)=V_{s}(x) \sin \left(\omega_{s} t\right) \\
\varphi_{s}(x, t)=\Phi_{s}(x) \sin \left(\omega_{s} t\right)
\end{array}\right.
$$

Using (3.23) in (3.10) and (3.11), we get:

$$
\rho A \omega_{s}^{2} V_{s}+k G A \frac{\partial^{2} V_{s}}{\partial x^{2}}-k G A \frac{\partial \Phi_{s}}{\partial x}=0
$$




$$
\rho I \omega_{s}^{2} \Phi_{s}+k G A \frac{\partial^{2} \Phi_{s}}{\partial x^{2}}-k G A \frac{\partial V_{s}}{\partial x}+k G A \Phi_{s}=0
$$

Combining (3.24) and (3.25) yields:

$$
\frac{\partial^{4} V_{s}}{\partial x^{4}}+\alpha_{s} \omega_{s}^{2} \frac{\partial^{2} V_{s}}{\partial x^{2}}+\left(\beta_{s} \omega_{s}^{4}-\gamma_{s} \omega_{s}^{2}\right) V_{s}=0
$$

where

$$
\left\{\begin{array}{l}
\alpha_{s}=\frac{\rho}{E}\left(1+\frac{E}{k G}\right) \\
\beta_{s}=\frac{\rho^{2}}{k E G} \\
\gamma_{s}=\frac{\rho A}{E I}
\end{array}\right.
$$

Similarly, for the curved part:

$$
\left\{\begin{array}{l}
u_{c}(\theta, t)=U_{c}(\theta) \sin \left(\omega_{c} t\right) \\
\varphi_{c}(\theta, t)=\Phi_{c}(\theta) \sin \left(\omega_{c} t\right)
\end{array}\right.
$$

From (3.28), (3.19) and (3.20) becomes:

$$
\begin{gathered}
\frac{\partial^{4} U_{c}}{\partial \theta^{4}}+\left(2-\frac{\rho R^{2}}{k G} \omega_{c}^{2}\right) \frac{\partial^{2} U_{c}}{\partial \theta^{2}}+\left(\frac{\rho R^{2}}{k G} \omega_{c}^{2}+1\right) U_{c}-R \Phi_{c}-R \frac{\partial^{2} \Phi_{c}}{\partial \theta^{2}}=0 \\
\frac{E I}{k A G R} \frac{\partial^{2} \Phi_{c}}{\partial \theta^{2}}-\left(\frac{\rho I R}{k A G} \omega_{c}^{2}+R\right) \Phi_{c}+\frac{\partial^{2} U_{c}}{\partial \theta^{2}}+U_{c}=0
\end{gathered}
$$

Combining (3.29) and (3.30) yields:

$$
\begin{aligned}
& \frac{\partial^{6} U_{c}}{\partial \theta^{6}}+\left(2+\alpha_{c}\left(\beta_{c}+\gamma_{c}\right) \omega_{c}^{2}\right) \frac{\partial^{4} U_{c}}{\partial \theta^{4}}+\left(1+\alpha_{c}\left(2 \beta_{c}-\gamma_{c}-1\right) \omega_{c}^{2}+\alpha_{c}^{2} \beta_{c} \gamma_{c} \omega_{c}^{4}\right) \frac{\partial^{2} U_{c}}{\partial \theta^{2}} \\
& +\left(\alpha_{c}\left(1+\beta_{c}\right) \omega_{c}^{2}-\alpha_{c}^{2} \beta_{c} \gamma_{c} \omega_{c}^{4}\right) U_{c}=0
\end{aligned}
$$

where

$$
\left\{\begin{aligned}
\alpha_{c} & =\frac{\rho A R^{4}}{E I} \\
\beta_{c} & =\frac{I}{A R^{2}} \\
\gamma_{c} & =\frac{E I}{k A G R^{2}}
\end{aligned}\right.
$$


In our case, we only look for the particular solution of the form $\pm \cos$ and $\pm \sin$ describing the standing wave inside the ring-type structure. For the next part, we chose those two following orthogonal particular solutions that respect the boundary conditions (3.21) and (3.22):

solution 1:

$$
\left\{A_{s_{1}} \cos \left(k_{s} x\right), A_{c_{1}} \cos \left(k_{c} \theta\right)\right\}
$$

and solution 2 :

$$
\left\{A_{s_{2}} \sin \left(k_{s} x\right), A_{c_{2}} \sin \left(k_{c} \theta\right)\right\}
$$

Substituting solution 1 (3.33) into equations (3.26) and (3.31) and boundary conditions (3.21) and (3.22) and remembering from the hypothesis of inextensibility of the curved beam (3.15) that $U_{c}=-\frac{\partial V_{c}}{\partial \theta}$ :

$$
\begin{gathered}
k_{s}^{4}-\alpha_{s} \omega_{s}^{2} k_{s}^{2}+\beta_{s} \omega_{s}^{4}-\gamma_{s} \omega_{s}^{2}=0 \\
k_{c}^{6}-\left(2+\alpha_{c}\left(\beta_{c}+\gamma_{c}\right) \omega_{c}^{2}\right) k_{c}^{4}+\left(1+\alpha_{c}\left(2 \beta_{c}-\gamma_{c}-1\right) \omega_{c}^{2}+\alpha_{c}^{2} \beta_{c} \gamma_{c} \omega_{c}^{4}\right) k_{c}^{2} \\
-\left(\alpha_{c}\left(1+\beta_{c}\right) \omega_{c}^{2}-\alpha_{c}^{2} \beta_{c} \gamma_{c} \omega_{c}^{4}\right)=0 \\
A_{s_{1}} \cos \left(k_{s} \frac{L}{2}\right)=A_{c_{1}} k_{c} \sin \left(k_{c} \frac{\pi}{2}\right) \\
A_{s_{1}} k_{s} \sin \left(k_{s} \frac{L}{2}\right)=R A_{c_{1}} k_{c}^{2} \cos \left(k_{c} \frac{\pi}{2}\right)
\end{gathered}
$$

Since the frequency of the straight part is equal to the frequency of the curved part (i.e. $\left.\omega_{s}=\omega_{c}=\omega\right)$, the dispersion equation of the straight beam (3.35) can be linked to the dispersion equation of the curved beam (3.36) by first calculating the frequency $\omega$ as a function of the wave number of the curved beam $k_{c}$ :

$$
A_{c} \omega^{4}+B_{c} \omega^{4}+C_{c}=0
$$

where 


$$
\left\{\begin{array}{l}
A_{c}=-\alpha_{c} \beta_{c} \gamma_{c}\left(k_{c}^{2}+1\right) \\
B_{c}=\alpha_{c}\left(\beta_{c}+\gamma_{c}\right) k_{c}^{4}-\alpha_{c}\left(2 \beta_{c}-\gamma_{c}-1\right) k_{c}^{2}+\alpha_{c}\left(1+\beta_{c}\right) \\
C_{c}=-k_{c}^{6}+2 k_{c}^{4}-k_{c}^{2}
\end{array}\right.
$$

which gives

$$
\omega^{2}\left(k_{c}\right)=\frac{-B_{c} \pm \sqrt{B_{c}^{2}-4 A_{c} C_{c}}}{2 A_{c}}
$$

and then calculating the corresponding wave number of the straight beam $k_{s}$ :

$$
k_{s}^{2}(\omega)=\frac{\alpha_{s} \omega^{2} \pm \sqrt{\alpha_{s}^{2} \omega^{4}-4\left(\beta_{s} \omega^{4}-\gamma_{s} \omega^{2}\right)}}{2}
$$

Now that the wave number of the straight part of the structure is linked to the wave number of the curved part, the modes corresponding to the solution 1 (3.33) are the modes that verify both boundary conditions (3.37) and (3.38). Since both boundary condition have the term $\frac{A_{s_{1}}}{k_{c} A_{c_{1}}}$ in common, a new boundary condition function $g_{1}\left(k_{c}\right)$ is created in (3.43):

$$
g_{1}\left(k_{c}\right)=\left(\frac{\sin \left(k_{c} \frac{\pi}{2}\right)}{\cos \left(k_{s}\left(k_{c}\right) \frac{L}{2}\right)}-R \frac{k_{c} \cos \left(k_{c} \frac{\pi}{2}\right)}{k_{s}\left(k_{c}\right) \sin \left(k_{s}\left(k_{c}\right) \frac{L}{2}\right)}\right)^{2}
$$

and the case of $g_{1}\left(k_{c}\right)=0$ means that both boundary conditions are satisfied, and thus $k_{c}$ is a valid wave number for a given length $L$ and radius $R$ of the ring-type structure.

As for the solution 2 (3.34), the equations that link $k_{s}$ to $k_{c}$ (equations (3.41) and (3.42)) are still valid however the boundary conditions change to:

$$
\begin{gathered}
A_{s_{2}} \sin \left(k_{s} \frac{L}{2}\right)=A_{c_{2}} k_{c} \cos \left(k_{c} \frac{\pi}{2}\right) \\
A_{s_{2}} k_{s} \cos \left(k_{s} \frac{L}{2}\right)=R A_{c_{2}} k_{c}^{2} \sin \left(k_{c} \frac{\pi}{2}\right)
\end{gathered}
$$

thus the boundary conditions function $g_{2}\left(k_{c}\right)$ is defined as $(3.46)$ :

$$
g_{2}\left(k_{c}\right)=\left(\frac{\cos \left(k_{c} \frac{\pi}{2}\right)}{\sin \left(k_{s}\left(k_{c}\right) \frac{L}{2}\right)}-R \frac{k_{c} \sin \left(k_{c} \frac{\pi}{2}\right)}{k_{s}\left(k_{c}\right) \cos \left(k_{s}\left(k_{c}\right) \frac{L}{2}\right)}\right)^{2}
$$




\subsubsection{Iterative design}

The objective of this analytical model is to optimally design the ring-type vibrator, i.e. to choose the correct radius that will allow a traveling wave in relation with the other given parameters. The input parameters are the length $L$ of the straight part, the width $w$ of the vibrator, the thickness $h$ of the vibrator, the mode number $n$ desired, the boundary values $R_{\min }$ and $R_{\max }$ of the radius and the material related parameters $E$, $\rho$ and $G$. Once those parameters are set, the main program consists of two embedded loops. The first loop set a value for the radius $R$ between its boundaries and in relation with its discretization, and then the boundary values of the wave number of the straight part is estimated by simply dividing the perimeter of the vibrator by the desired mode, or $k_{c_{\text {low }}}=\frac{2 \pi}{\frac{2 \pi R+2 L}{n-1}}$ and $k_{c_{\text {high }}}=\frac{2 \pi}{\frac{2 \pi R+2 L}{n+1}}$. The second loop, which is embedded in the first, calculates the values of the boundary functions $g_{1}$ and $g_{2}$ for the values of $k_{c}$ between its boundaries and in function of its discretization, and the value of the frequency for the minimum of $g_{1}$ and $g_{2}$ is then saved in order to later find the cases when those frequencies are equals. The MatLab program used for the iterative design is available in appendix B.3.

A example plot of functions $g_{1}(\omega)$ and $g_{2}(\omega)$ for an aluminium alloy and $\mathrm{h}=3 \mathrm{~mm}$, $\mathrm{w}=50 \mathrm{~mm}, \mathrm{~L}=220 \mathrm{~mm}, \mathrm{R}=50 \mathrm{~mm}$ is presented fig. 3.7 .

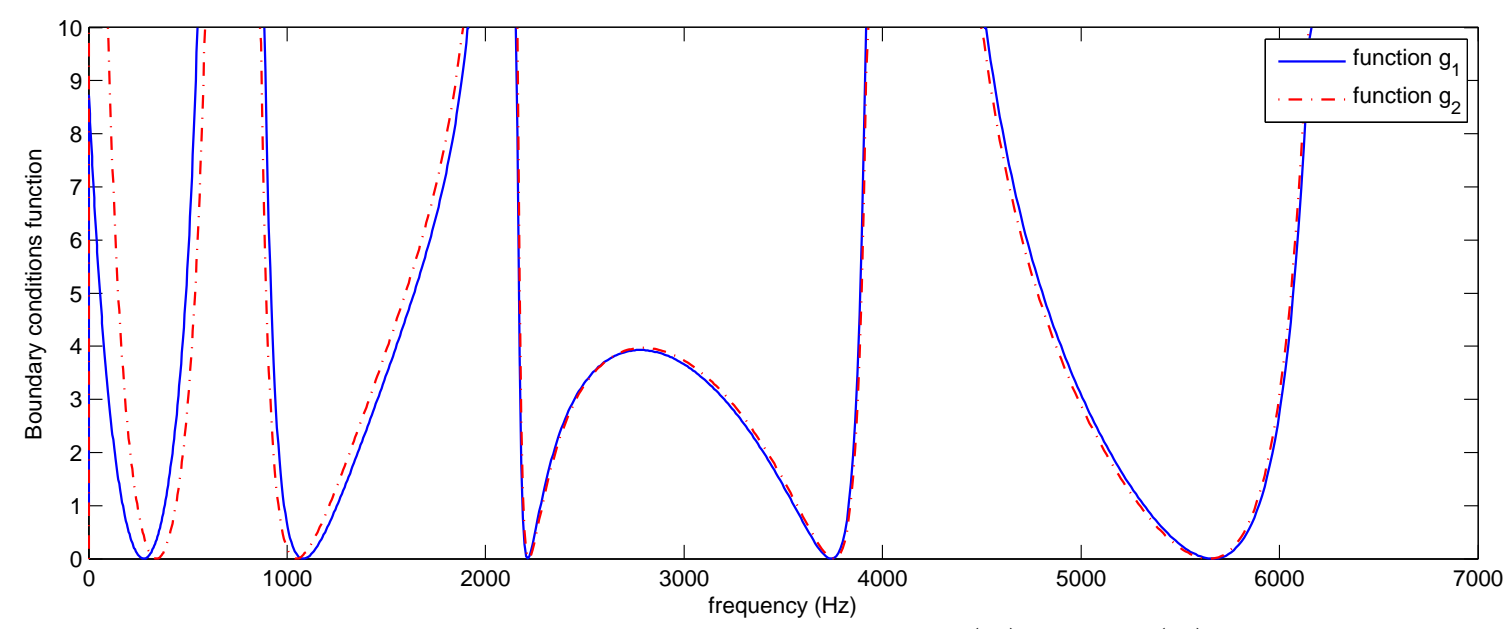

Figure 3.7: Plot of the functions $g_{1}(\omega)$ and $g_{2}(\omega)$.

and both solutions are represented in fig. 3.8 where solution 1 has a frequency of $5666 \mathrm{~Hz}$ and solution 2 5654Hz.: 


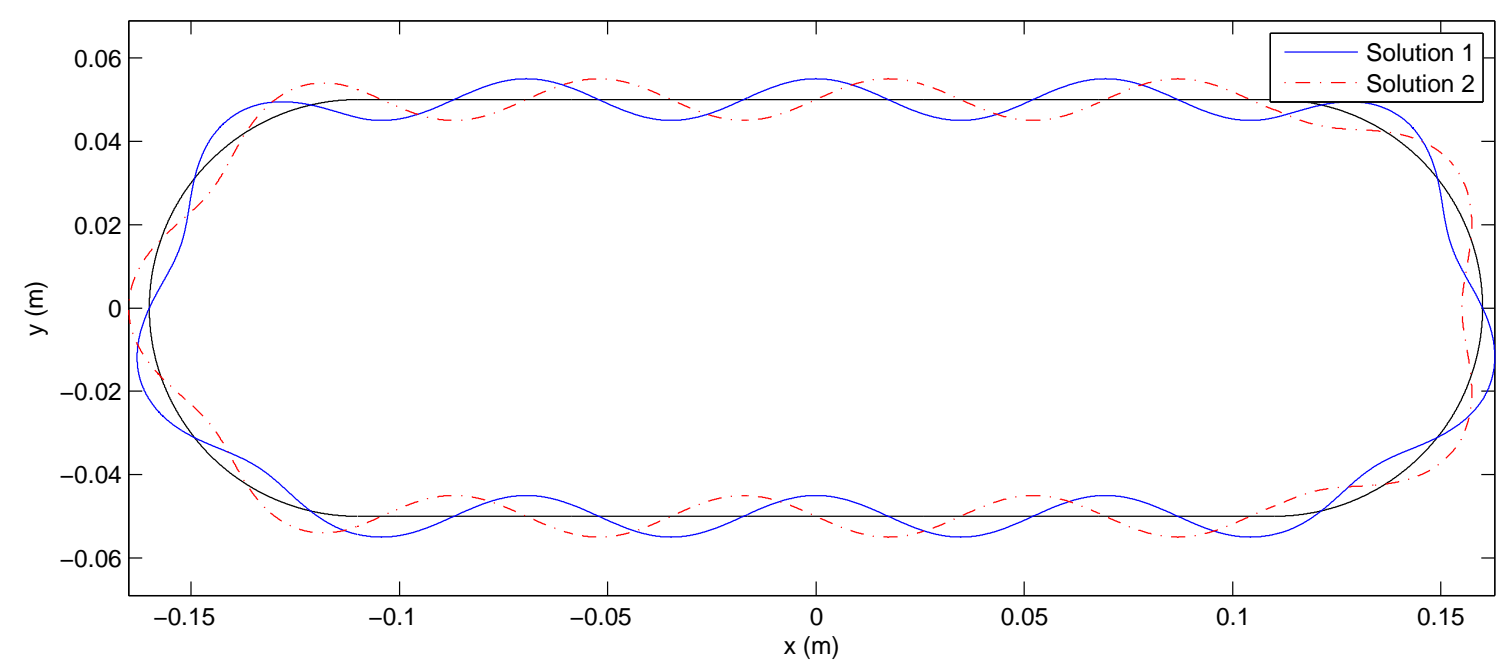

Figure 3.8: Plot of the solution 1 and solution 2 . 


\section{$4 \quad$ Numerical modeling}

In this chapter, the modeling of a beam vibrator (of the same kind that (KOYAMA; NAKAMURA, 2010b)), the modeling of the proposed ring-type vibrator and the modeling of the acoustic field in this devices are presented. The goal of this project is to evaluate an alternative solution for the vibrator, where the distance traveled by the particle is linear and where the particle can be moved from one system to another easily. Therefore, the use of a ring-type structure as vibrator is discussed here.

\subsection{Finite element modeling of the beam vibrator}

This section presents the modeling of vibrator of the same type as described by D. Koyama and K. Nakamura (KOYAMA; NAKAMURA, 2010b), i.e. using a flexural vibration mode in a plate as an acoustic transducer to generate the acoustic field. In the model presented here, the solid (vibrating plate) will generate an acoustic field, however the hypothesis is made that the resulting acoustic field will not impact the solid. This hypothesis is justified by the fact that the acoustic field occurs in air, which has a low density compared to the solid, and thus has a negligible impact on it.

\subsubsection{Finite element formulation}

In the system described in (KOYAMA; NAKAMURA, 2010b) (fig. 4.1a), the acoustic transducer is a $605 \times 60 \times 3 \mathrm{~mm}$ plate vibrating at its $33^{\text {rd }}$ flexural mode. Only the flexural modes are useful to generate the acoustic field desired to levitate particles and therefore the vibrating plate can, in that case, be modeled as a beam. Also, since the plate is modeled as a beam, and the contact surface between the transducers and the plate is very small compared to the total length of the beam, the transducers are modeled as two ponctual loads $F_{1}$ and $F_{2}$, at the postion $\ell_{1}$ and $\ell_{2}$, respectively. The model is represented in fig. $4.1 \mathrm{~b}$.

The beam has no boundary conditions (free-free beam) and the Euler-Bernoulli beam theory will be used to perform the finite element modeling. The Euler-Bernoulli beam 

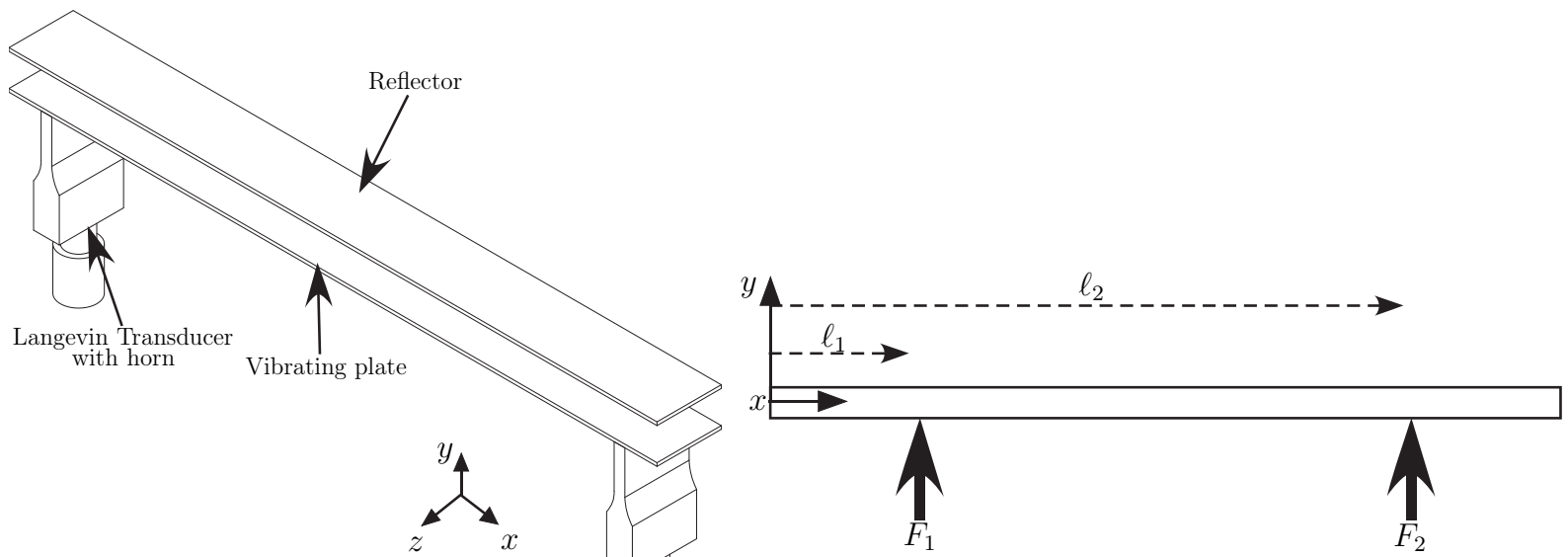

(a)

(b)

Figure 4.1: (a) Acoustic levitation transportation system as presented in (KOYAMA; NAKAMURA, 2010b) (b) Beam model of the vibrating plate.

theory assumes that the plane cross sections perpendicular to the axis of the beam remain plane and perpendicular to the axis after deformation. At a point of a beam represented in fig. 4.2, the forces and their interrelationships are:

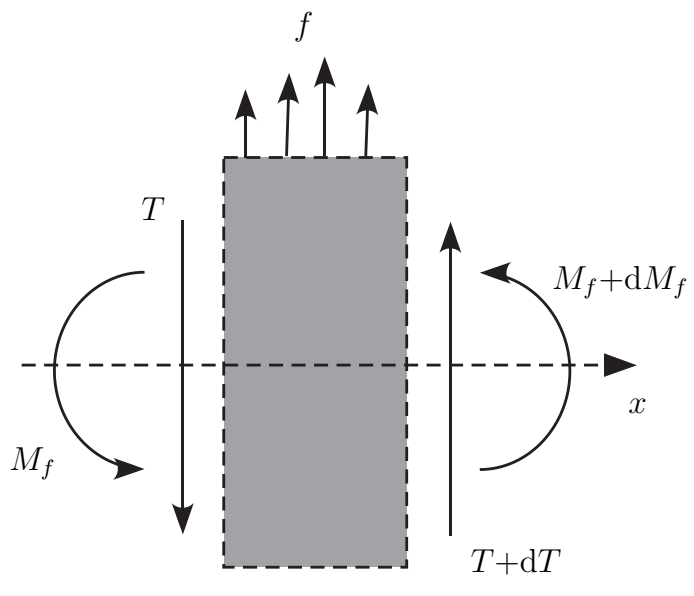

$\mathrm{d} x$

Figure 4.2: Forces and moment at a point of the beam.

$$
\left\{\begin{array}{l}
T+\mathrm{d} T-T+f \mathrm{~d} x=\rho A \frac{\partial^{2} v}{\partial t^{2}} \mathrm{~d} x \\
(T+\mathrm{d} T) \frac{\mathrm{d} x}{2}+M_{f}+\mathrm{d} M_{f}-M_{f}+T \frac{\mathrm{d} x}{2}=0 \\
M_{f}=E I_{z} \frac{\partial^{2} v}{\partial x^{2}}
\end{array}\right.
$$

The transverse deflection $v$ of the beam is governed by the following differential equation:

$$
\left.\rho A \frac{\partial^{2} v}{\partial t^{2}}+E I_{z} \frac{\partial^{4} v}{\partial t^{4}}=f, \forall x \in\right] 0, \ell[
$$


where $\ell$ is the length of the beam, $\rho$ is the density of the beam, $A$ the area of the beam, $E$ the Young modulus of the beam, $I_{z}$ the second moment of area defined as $I_{z}=\frac{b h^{3}}{12}$, where $b$ and $h$ are the width and thickness of the beam, respectively, and $f$ is the distributed transverse load (here $f=0$ ). Because the equation 4.2 is a fourth-order differential equation, $v$ needs to satisfy four boundary conditions and since the model is a free-free beam, the boundary conditions are:

$$
\left\{\begin{array}{l}
\frac{\partial^{2} v}{\partial x^{2}}=0 \text { at } x=0 \text { and } x=\ell \\
\frac{\partial^{3} v}{\partial x^{3}}=0 \text { at } x=0 \text { and } x=\ell
\end{array}\right.
$$

The element used for the discretization is shown in fig. 4.3.

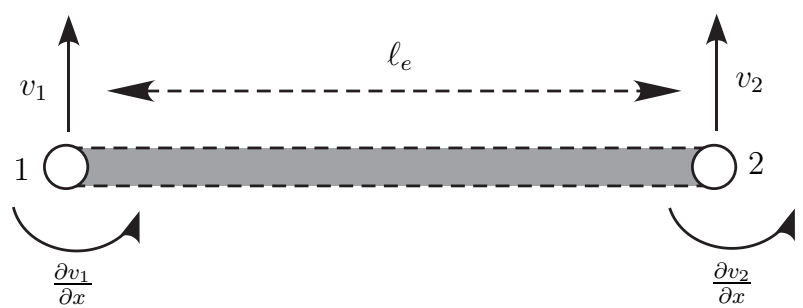

Figure 4.3: $1 \mathrm{D}$ element of a beam.

Considering an homogenous beam $\left(E, A, I_{z}\right.$ and $\rho$ constants) and no transversal loads $(f=0)$, applying Galerkin's method gives (4.4):

$$
\begin{gathered}
\int_{0}^{\ell_{e}} \phi\left[\rho A \frac{\partial^{2} v}{\partial t^{2}}+E I_{z} \frac{\partial^{4} v}{\partial x^{4}}\right] \mathrm{d} x=0 \\
\rho A \int_{0}^{\ell_{e}} \phi \frac{\partial^{2} v}{\partial t^{2}} \mathrm{~d} x+E I_{z}\left(\left[\phi \frac{\partial^{3} v}{\partial x^{3}}\right]_{0}^{\ell_{e}}-\int_{0}^{\ell_{e}} \frac{\partial \phi}{\partial x} \frac{\partial^{3} v}{\partial x^{3}} \mathrm{~d} x\right)=0 \\
\rho A \int_{0}^{\ell_{e}} \phi \frac{\partial^{2} v}{\partial t^{2}} \mathrm{~d} x+E I_{z}\left(\left[\phi \frac{\partial^{3} v}{\partial x^{3}}\right]_{0}^{\ell_{e}}-\left[\frac{\partial \phi}{\partial x} \frac{\partial^{2} v}{\partial x^{2}}\right]_{0}^{\ell_{e}}+\int_{0}^{\ell_{e}} \frac{\partial^{2} \phi}{\partial x^{2}} \frac{\partial^{2} v}{\partial x^{2}} \mathrm{~d} x\right)=0
\end{gathered}
$$

where $\phi$ represents the interpolation functions. Seing in the equation 4.1 that $E I_{z} \frac{\partial^{2} v}{\partial x^{2}}=$ $M_{f}$ and $E I_{z} \frac{\partial^{3} v}{\partial x^{3}}=T$, the weak form of 4.2 can be written as:

$\rho A \int_{0}^{\ell_{e}} \phi \frac{\partial^{2} v}{\partial t^{2}} \mathrm{~d} x+E I_{z} \int_{0}^{\ell_{e}} \frac{\partial^{2} \phi}{\partial x^{2}} \frac{\partial^{2} v}{\partial x^{2}} \mathrm{~d} x=-\phi(0) T(0)-\phi\left(\ell_{e}\right) T\left(\ell_{e}\right)+\frac{\partial \phi}{\partial x}(0) M f(0)+\frac{\partial \phi}{\partial x}\left(\ell_{e}\right) M f\left(\ell_{e}\right)$

The equation 4.7 shows that the interpolation functions have to be at least a four parameters polynomial. The interpolation functions chosen are the Hermite interpolation functions: 


$$
\begin{gathered}
\forall x \in\left[0, \ell_{e}\right], \\
\phi_{1}=1-3\left(\frac{x}{\ell_{e}}\right)^{2}+2\left(\frac{x}{\ell_{e}}\right)^{3} \\
\phi_{2}=-x\left(1-\frac{x}{\ell_{e}}\right)^{2} \\
\phi_{3}=3\left(\frac{x}{\ell_{e}}\right)^{2}-2\left(\frac{x}{\ell_{e}}\right)^{3} \\
\phi_{4}=-x\left[\left(\frac{x}{\ell_{e}}\right)^{2}-\frac{x}{\ell_{e}}\right]
\end{gathered}
$$

By posing:

$$
\begin{gathered}
N=\left\{\phi_{1}, \phi_{2}, \phi_{3}, \phi_{4}\right\} \\
v=N V_{e}, \phi=N \Phi \\
\frac{\partial^{2} N}{\partial x^{2}}=\left\{\frac{\partial^{2} \phi_{1}}{\partial x^{2}}, \frac{\partial^{2} \phi_{2}}{\partial x^{2}}, \frac{\partial^{2} \phi_{3}}{\partial x^{2}}, \frac{\partial^{2} \phi_{4}}{\partial x^{2}}\right\} \\
\frac{\partial^{2} v}{\partial x^{2}}=\frac{\partial^{2} N}{\partial x^{2}} V_{e}, \frac{\partial^{2} \phi}{\partial x^{2}}=\frac{\partial^{2} N}{\partial x^{2}} \Phi \\
V_{e}=\left\{v_{1}, \frac{\partial v_{1}}{\partial x}, v_{2}, \frac{\partial v_{2}}{\partial x}\right\}^{T}, \Phi=\left\{\phi_{1}, \frac{\partial \phi_{1}}{\partial x}, \phi_{2}, \frac{\partial \phi_{2}}{\partial x}\right\}^{T}
\end{gathered}
$$

the equation 4.7 can be written as:

$$
\mathbf{M}_{\mathbf{e}} \frac{\partial^{2} V_{e}}{\partial t^{2}}+\mathbf{K}_{\mathbf{e}} V_{e}=F_{e}
$$

where

$$
\begin{gathered}
\mathbf{M}_{\mathbf{e}}=\rho A \int_{0}^{\ell_{e}} N^{t} N \mathrm{~d} x=\frac{\rho A \ell_{e}}{420}\left[\begin{array}{cccc}
156 & -22 \ell_{e} & 54 & 13 \ell_{e} \\
-22 \ell_{e} & 4 \ell_{e}^{2} & -13 \ell_{e} & -3 \ell_{e}^{2} \\
54 & -13 \ell_{e} & 156 & 22 \ell_{e} \\
13 \ell_{e} & -3 \ell_{e}^{2} & 22 \ell_{e} & 4 \ell_{e}^{2}
\end{array}\right] \\
\mathbf{K}_{\mathbf{e}}=E I_{z} \int_{0}^{\ell_{e}} \frac{\partial^{2} N^{T}}{\partial x^{2}} \frac{\partial^{2} N}{\partial x^{2}} \mathrm{~d} x=\frac{2 E I_{z}}{\ell_{e}^{3}}\left[\begin{array}{cccc}
6 & -3 \ell_{e} & -6 & -3 \ell_{e} \\
-3 \ell_{e} & 2 \ell_{e}^{2} & 3 \ell_{e} & \ell_{e}^{2} \\
-6 & 3 \ell_{e} & 6 & 3 \ell_{e} \\
-3 \ell_{e} & \ell_{e}^{2} & 3 \ell_{e} & 2 \ell_{e}^{2}
\end{array}\right] \\
F_{e}=\left\{-T(0), M_{f}(0),-T\left(\ell_{e}\right), M_{f}\left(\ell_{e}\right)\right\}^{T}
\end{gathered}
$$

Once all the elements of the beam are assembled, the equation is:

$$
\mathbf{M} \frac{\partial^{2} V}{\partial t^{2}}+\mathbf{K} V=F
$$




\subsubsection{Modal analysis}

A modal analysis of the beam is useful to identify the vibration modes of the plate and their frequency. Since in the case of a modal analysis the variables are harmonic functions, $\mathrm{V}$ can be written as $V(t)=V \cos (\omega t)=R e\left(V e^{j \omega t}\right)$, and there are no loads: $F=0$. Accordingly, the equation 4.9 can be rewritten as an eigenvalue problem:

$$
\left(\mathbf{K}-\omega_{n}^{2} \mathbf{M}\right) V_{n}=0
$$

where $\omega_{n}$ is the angular frequency of the $n^{\text {th }}$ mode of the beam and $V_{n}$ represents the $n^{t h}$ mode shape of the beam.

\subsubsection{Harmonic analysis}

An harmonic analysis of the model fig. $4.1 \mathrm{~b}$ is needed to simulate the behavior of the vibrating plate when the two langevin transducers are operating. The two transducers (represented as $F_{1}$ and $F_{2}$ in the model) will be working at the angular frequency $\omega$ and so:

$$
\begin{gathered}
F_{1}(t)=F_{1} \cos \left(\omega t+\varphi_{1}\right)=\operatorname{Re}\left(\hat{F}_{1} e^{j \omega t}\right), \hat{F}_{1}=F_{1} e^{j \varphi_{1}} \\
F_{2}(t)=F_{2} \cos \left(\omega t+\varphi_{2}\right)=\operatorname{Re}\left(\hat{F}_{2} e^{j \omega t}\right), \hat{F}_{2}=F_{2} e^{j \varphi_{2}} \\
V(t)=V \cos (\omega t+\psi)=\operatorname{Re}\left(\hat{V} e^{j \omega t}\right), \hat{V}=V e^{j \psi}
\end{gathered}
$$

where $\hat{F}_{1}, \hat{F}_{2}$ and $\hat{V}$ are complex variables with phases equals to $\varphi_{1}, \varphi_{2}$ and $\psi$, respectively. We will also be introducing damping in our model in order to consider the natural damping of the material of the model. The equation 4.9 including damping is:

$$
\mathbf{M} \frac{\partial^{2} \hat{V}}{\partial t^{2}}+\mathbf{C} \frac{\partial \hat{V}}{\partial t}+\mathbf{K} \hat{V}=\hat{F}
$$

where $\mathbf{C}$ is the Rayleigh damping matrix defined by (COOK et al., 2002):

$$
\mathbf{C}=\alpha \mathbf{M}+\beta \mathbf{K}
$$

where $\alpha$ and $\beta$ are two coefficient depending of the material. The corresponding damping ratio is:

$$
\xi=\frac{1}{2}\left(\frac{\alpha}{\omega}+\beta \omega\right)
$$


The damped natural frequency $f_{d}$ is related to the undamped natural frequency $f_{n}$ by:

$$
f_{d}=f_{n} \sqrt{1-\xi^{2}}
$$

Finally, the equation of the harmonic analysis is:

$$
\left((1+\beta j \omega) \mathbf{K}-\left(\omega^{2}-\alpha j \omega\right) \mathbf{M}\right) \hat{V}=\hat{F}
$$

\subsubsection{MatLab implementation}

The finite element model developed in the previous section is implemented in the numerical computing software MatLab developed by MathWorks. The full program is detailed in appendix A.1.

\subsection{Finite element modeling of the ring-type vibrator}

The preceding analytical modeling made in chapter 3 will give a very good idea to which ratio ${ }^{R} / L$ will yield two orthogonal modes at the same frequency, however due to the rather large width needed for the acoustic levitation ((KOYAMA; NAKAMURA, 2010b) used a $60 \mathrm{~mm}$ wide plate), the beam theory might not be the most reliable here. Therefore, a finite element modeling using 3D solid elements is made here using the commercial software ANSYS for the simulations.

The model is made by using the ANSYS Parametric Design Language (APDL), as it allows a great flexibility and facilitates interaction between ANSYS and MatLab. The geometry is fully parameterized in order to do iterative design later on, and is then discretized by using the ANSYS eight nodes and three degrees of freedom element SOLID185 (see fig.4.4). 

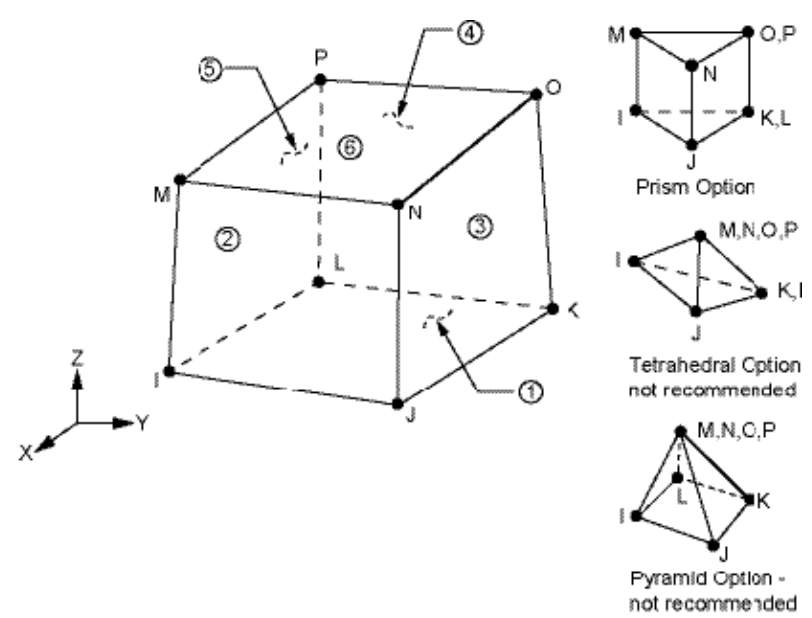

Figure 4.4: ANSYS element SOLID185.

The mesh size of the ring-type structure is chosen as $1 \mathrm{~mm}$. The $3 \mathrm{D}$ solid ring-type structure representation in ANSYS is shown in fig.4.5.

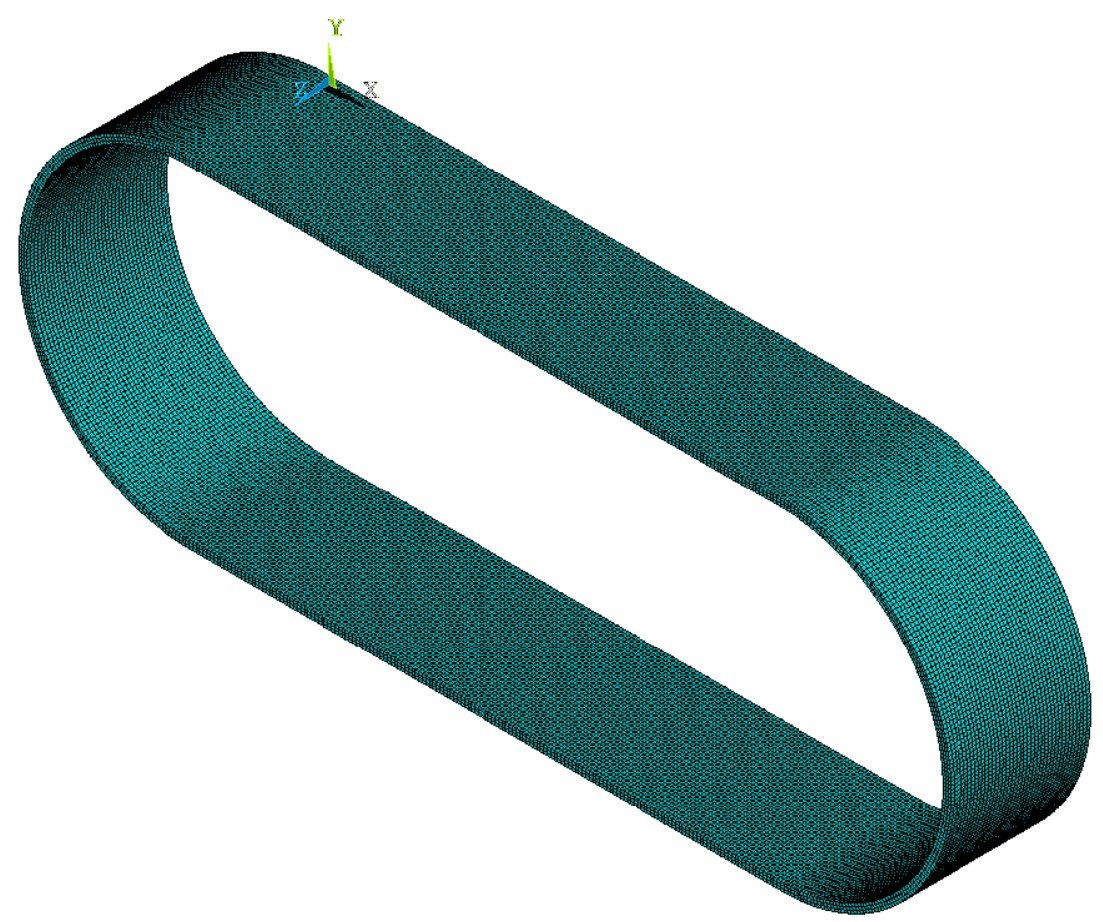

Figure 4.5: 3D solid model of the ring type structure.

\subsection{Finite element modeling of the acoustical horns}

Following the article by (AMIN; AHMED; YOUSSEF, 1995), as detailed in section 2.3.3, models of two profiles of acoustical horn are made, as shown in fig.4.6. The models are made by using the ANSYS APDL with a fully parameterized geometry, and discretized with the SOLID185 element detailed in $§ 4.2$. 

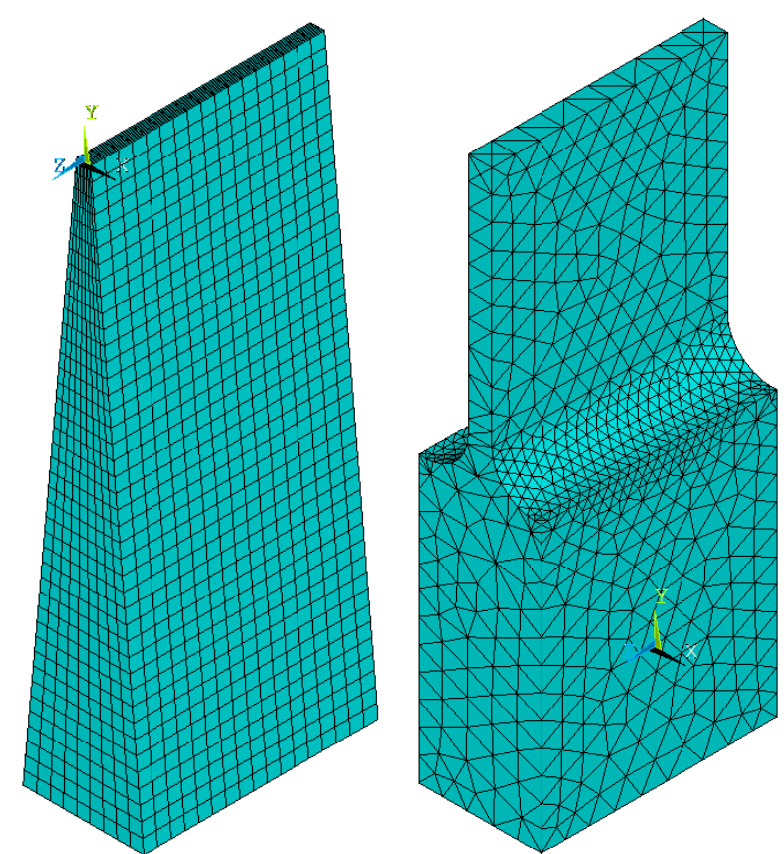

Figure 4.6: 3D solid model of the horns (left: conical profile horn; right: stepped profile horn).

\subsection{Finite element modeling of the acoustic transducers}

In order to design properly the Langevin-type acoustic transducers, modal analysis must be made separately on both the transducer and its mechanical amplifier. Then, harmonic analysis considering the whole transducer are made in order to tune the design and ensure that the manufactured transducer have a working frequency very close of that desired.

As before, the model is made by using the ANSYS APDL with a fully parameterized geometry, and discretized with the twenty nodes and up to five degrees of freedom SOLID226 element (see fig.4.7), as it allows coupled structural/piezoelectric analysis.
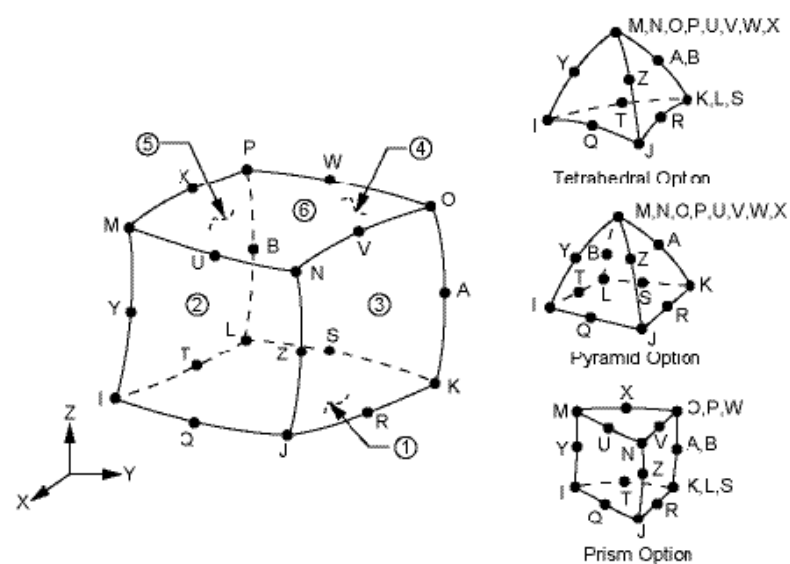

Figure 4.7: ANSYS element SOLID226.

The model consider the fillets, holes for the screws, and only one fourth of the geometry 
is modeled with symmetry constraints on both faces in order to simulate the whole transducer. Due to the complexity induced by the fillets into the geometry, the mesh here is created automatically by ANSYS using the SMRTSIZE function (set to 2, the second finest parameter). The models in ANSYS for the transducer, the amplifier and the transducer combined with its amplifier are shown fig.4.8.

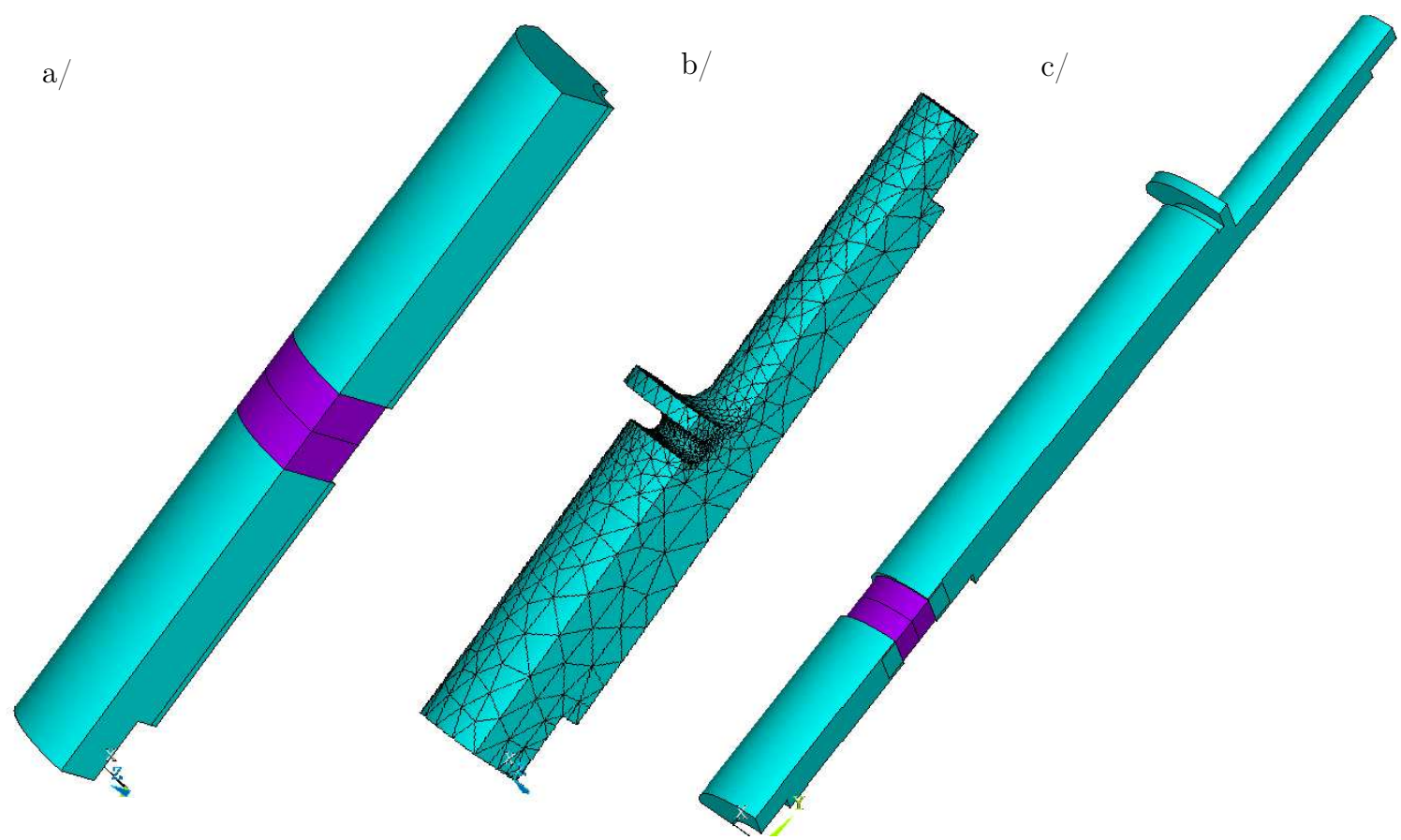

Figure 4.8: a/3D solid model of the Langevin transducer (piezoelectric ceramics in purple); b/3D solid model of mechanical amplifier; $\mathrm{c} / 3 \mathrm{D}$ solid model of the complete acoustic transducer (piezoelectric ceramics in purple).

\subsection{Modeling of the acoustic field}

\subsubsection{D model}

The acoustic field represents the behavior of the air between the vibrating plate and the reflector. The model used is a $2 \mathrm{D}$ plane, represented in fig. 4.9, and the hypothesis that the the acoustic field will not influence the vibration of the beam is made. The boundary conditions are a total transmission of node displacement from the beam to the acoustic field where they are in contact, and total reflection of the sound wave for both sides. The lateral boundary conditions are infinite, non-reflecting boundaries.

The acoustic field will be a response of the harmonic excitation exerted by the beam, hence will be itself harmonic and may be represented as

$$
p(x, y, t)=p_{a}(x, y) \cos (w t+\psi)=\operatorname{Re}\left(p_{a} e^{j(\omega t+\psi)}\right)=\operatorname{Re}\left(\hat{p} e^{j \omega t}\right)
$$




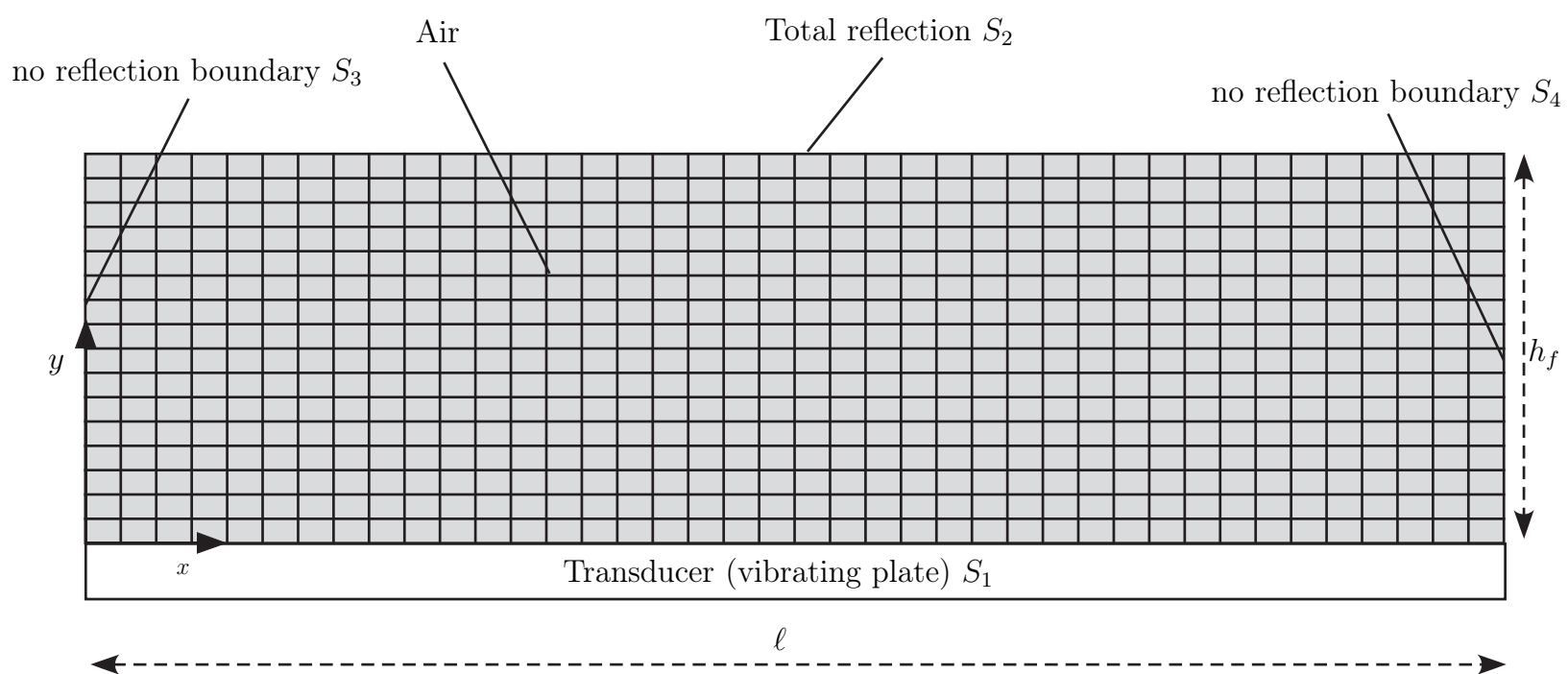

Figure 4.9: Model of the acoustic field.

where $\hat{p}$ is the complex amplitude of the pressure. Substituting eq. 4.16 to eq. 2.1 results in the Helmholtz equation:

$$
\nabla^{2} \hat{p}+k^{2} \hat{p}=0
$$

where $k$ is the wave number defined as $k=\frac{\omega}{c}$.

\section{a) Finite element formulation}

In two dimension the equation 4.17 becomes:

$$
\frac{\partial^{2} \hat{p}}{\partial x^{2}}+\frac{\partial^{2} \hat{p}}{\partial y^{2}}+k^{2} \hat{p}=0
$$

The weak form of the Helmholtz equation 4.18 for a 2D element of area $A_{e}$ and contour $S_{e}$ is developed by using Galerkin's method:

$$
\int_{A_{e}} \phi\left[\frac{\partial^{2} \hat{p}}{\partial x^{2}}+\frac{\partial^{2} \hat{p}}{\partial y^{2}}+k^{2} \hat{p}\right] \mathrm{d} A=0
$$

where $\phi$ represent the interpolation functions. Integrating by parts gives:

$$
\begin{aligned}
\int_{A_{e}} \phi \frac{\partial^{2} \hat{p}}{\partial x^{2}} \mathrm{~d} A & =\int_{A_{e}} \frac{\partial}{\partial x}\left(\phi \frac{\partial \hat{p}}{\partial x}\right) \mathrm{d} A-\int_{A_{e}} \frac{\partial \phi}{\partial x} \frac{\partial \hat{p}}{\partial x} \mathrm{~d} A \\
\int_{A_{e}} \phi \frac{\partial^{2} \hat{p}}{\partial y^{2}} \mathrm{~d} A & =\int_{A_{e}} \frac{\partial}{\partial y}\left(\phi \frac{\partial \hat{p}}{\partial y}\right) \mathrm{d} A-\int_{A_{e}} \frac{\partial \phi}{\partial y} \frac{\partial \hat{p}}{\partial y} \mathrm{~d} A
\end{aligned}
$$

and using Stokes' theorem: 


$$
\begin{aligned}
& \int_{A_{e}} \frac{\partial}{\partial x}\left(\phi \frac{\partial \hat{p}}{\partial x}\right) \mathrm{d} A=\oint_{S_{e}} \phi \frac{\partial \hat{p}}{\partial x} \cdot n_{x} \mathrm{~d} S \\
& \int_{A_{e}} \frac{\partial}{\partial y}\left(\phi \frac{\partial \hat{p}}{\partial y}\right) \mathrm{d} A=\oint_{S_{e}} \phi \frac{\partial \hat{p}}{\partial y} \cdot n_{y} \mathrm{~d} S
\end{aligned}
$$

therefore, the weak form of 4.18 is:

$$
\int_{A_{e}}\left(\frac{\partial \phi}{\partial x} \frac{\partial \hat{p}}{\partial x}+\frac{\partial \phi}{\partial x} \frac{\partial \hat{p}}{\partial y}\right) \mathrm{d} A-k^{2} \int_{A_{e}} \phi \hat{p} \mathrm{~d} A=\oint_{S_{e}} \phi \nabla \hat{p} \cdot n \mathrm{~d} S
$$

where $n$ is the vector normal to the boundary.

The element used for the discretization is a four-node quadrilateral as shown in fig. 4.10 .

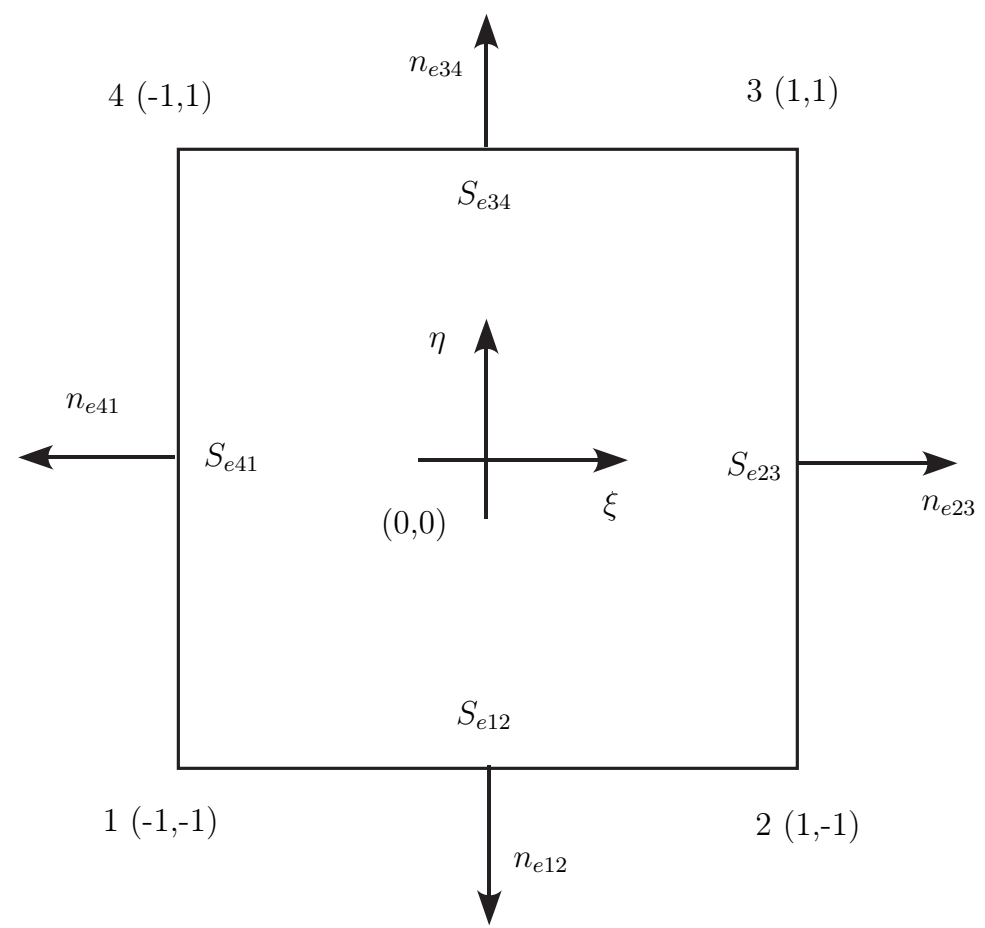

Figure 4.10: Four-node quad element.

The four shape functions of the four-node quad element can be written as:

$$
\begin{aligned}
& N_{1}=\frac{1}{4}(1-\xi)(1-\eta) \\
& N_{2}=\frac{1}{4}(1+\xi)(1-\eta) \\
& N_{3}=\frac{1}{4}(1+\xi)(1+\eta) \\
& N_{4}=\frac{1}{4}(1-\xi)(1+\eta)
\end{aligned}
$$

The relation between the local coordinates $(\xi, \eta)$ and the global coordinate $(x, y)$ is: 


$$
\left\{\begin{array}{l}
x=N_{1} x_{1}+N_{2} x_{2}+N_{3} x_{3}+N_{4} x_{4} \\
y=N_{1} y_{1}+N_{2} y_{2}+N_{3} y_{3}+N_{4} y_{4}
\end{array}\right.
$$

and for a function $f(x, y)$

$$
\left\{\begin{array}{l}
\frac{\partial f}{\partial x} \\
\frac{\partial f}{\partial y}
\end{array}\right\}=\mathbf{J}^{-1}\left\{\begin{array}{l}
\frac{\partial f}{\partial \xi} \\
\frac{\partial f}{\partial \eta}
\end{array}\right\}
$$

where $\mathbf{J}$ is the Jacobian matrix defined as:

$$
\mathbf{J}=\left[\begin{array}{ll}
\frac{\partial x}{\partial \xi} & \frac{\partial y}{\partial \xi} \\
\frac{\partial x}{\partial \eta} & \frac{\partial y}{\partial \eta}
\end{array}\right]
$$

Another useful result is:

$$
\mathrm{d} x \mathrm{~d} y=\operatorname{det}(\mathbf{J}) \mathrm{d} \xi \mathrm{d} \eta
$$

By posing:

$$
\begin{gathered}
N=\left\{N_{1}, N_{2}, N_{3}, N_{4}\right\} \\
\hat{p}=N \hat{P}_{e}, \phi=N \Phi \\
\frac{\partial N}{\partial x}=\left\{\frac{\partial N_{1}}{\partial x}, \frac{\partial N_{2}}{\partial x}, \frac{\partial N_{3}}{\partial x}, \frac{\partial N_{4}}{\partial x}\right\}, \frac{\partial N}{\partial y}=\left\{\frac{\partial N_{1}}{\partial y}, \frac{\partial N_{2}}{\partial y}, \frac{\partial N_{3}}{\partial y}, \frac{\partial N_{4}}{\partial y}\right\} \\
\frac{\partial \hat{p}}{\partial x}=\frac{\partial N}{\partial x} \hat{P}_{e}, \frac{\partial \phi}{\partial x}=\frac{\partial N}{\partial x} \Phi \\
\frac{\partial \hat{p}}{\partial y}=\frac{\partial N}{\partial y} \hat{P}_{e}, \frac{\partial \phi}{\partial y}=\frac{\partial N}{\partial y} \Phi \\
\hat{P}_{e}=\left\{\hat{p}_{1}, \hat{p_{2}}, \hat{p_{3}}, \hat{p_{4}}\right\}^{T}, \Phi=\left\{\phi_{1}, \phi_{2}, \phi_{3}, \phi_{4}\right\}^{T}
\end{gathered}
$$

the equation 4.20 can be written as:

$$
\left(\mathbf{H}_{\mathbf{e}}-k^{2} \mathbf{Q}_{\mathbf{e}}\right) \hat{P}_{e}=\hat{F}_{e}
$$


where

$$
\begin{gathered}
\mathbf{H}_{\mathbf{e}}=\int_{A_{e}}\left(\frac{\partial N^{T}}{\partial x} \frac{\partial N}{\partial x}+\frac{\partial N^{T}}{\partial y} \frac{\partial N}{\partial y}\right) \mathrm{d} x \mathrm{~d} y \\
\mathbf{Q}_{\mathbf{e}}=\int_{A_{e}} N^{T} N \mathrm{~d} x \mathrm{~d} y \\
\hat{F}_{e}=-\oint_{S_{e 12}} N \frac{\partial \hat{p}}{\partial y} \mathrm{~d} S+\oint_{S_{e 23}} N \frac{\partial \hat{p}}{\partial x} \mathrm{~d} S+\oint_{S_{e 34}} N \frac{\partial \hat{p}}{\partial y} \mathrm{~d} S-\oint_{S_{e 41}} N \frac{\partial \hat{p}}{\partial x} \mathrm{~d} S
\end{gathered}
$$

Once all the elements of the fluid are assembled, the equation is:

$$
\left.\left(\mathbf{H}-k^{2} \mathbf{Q}\right]\right) \hat{P}=\hat{F}
$$

\section{b) Boundary conditions}

The vector $\hat{F}$ is defined by:

$$
\hat{F}=-\oint_{S_{1}} N \frac{\partial \hat{p}}{\partial y} \mathrm{~d} S+\oint_{S_{2}} N \frac{\partial \hat{p}}{\partial x} \mathrm{~d} S+\oint_{S_{3}} N \frac{\partial \hat{p}}{\partial y} \mathrm{~d} S-\oint_{S_{4}} N \frac{\partial \hat{p}}{\partial x} \mathrm{~d} S
$$

where $S_{1}, S_{2}, S_{3}$ and $S_{4}$ are defined in fig. 4.9 .

The natural Neumann boundary condition $\frac{\partial \hat{P}}{\partial n}=0$ is a total reflection boundary, as a total reflection boundary condition is defined by $u=0$ (IHLENBURG, 1998) and the Euler equation 2.2 links the pressure gradient to the particle speed. Therefore, the boundary $S_{2}$ will be free.

Considering a total movement transmission between the beam and the acoustic field, and by applying Euler relation defined by 2.2 , on $S_{1}$ :

$$
\hat{F}_{1}=-j \omega \rho_{0} \oint_{S_{1}} N \hat{v} \mathrm{~d} S
$$

where $\hat{v}$ is the complex normal velocity of the flexural vibrations of the beam.

The non-reflecting boundaries on $S_{3}$ and $S_{4}$ correspond to an infinite space, where the wave will slowly be damped. One solution to simulate this effect in finite element is to use an absorbing boundary layer adjacent to the edge of the finite element mesh, in which the acoustic wave will decay exponentially. This technique is called perfectly matched layers (PML) and was first introduced by (BERENGER, 1996). The idea is that inside the PMLs, the differential equation is transformed as (JOHNSON, 2008): 


$$
\begin{gathered}
\frac{\partial}{\partial x} \rightarrow \frac{1}{1+j \frac{\sigma_{x}(x)}{\omega}} \frac{\partial}{\partial x} \\
\frac{\partial}{\partial y} \rightarrow \frac{1}{1+j \frac{\sigma_{y}(y)}{\omega}} \frac{\partial}{\partial y}
\end{gathered}
$$

where $\sigma_{x}(x)$ and $\sigma_{y}(y)$ can be constant, quadratic or cubic functions. The PML absorbing boundaries have no physical meaning, being only a technique to simulate an infinite, non-reflecting boundary condition for the Helmholtz equation (eq. 4.17), and all results inside the PMLs are to be discarded. The model in fig. 4.9 become:

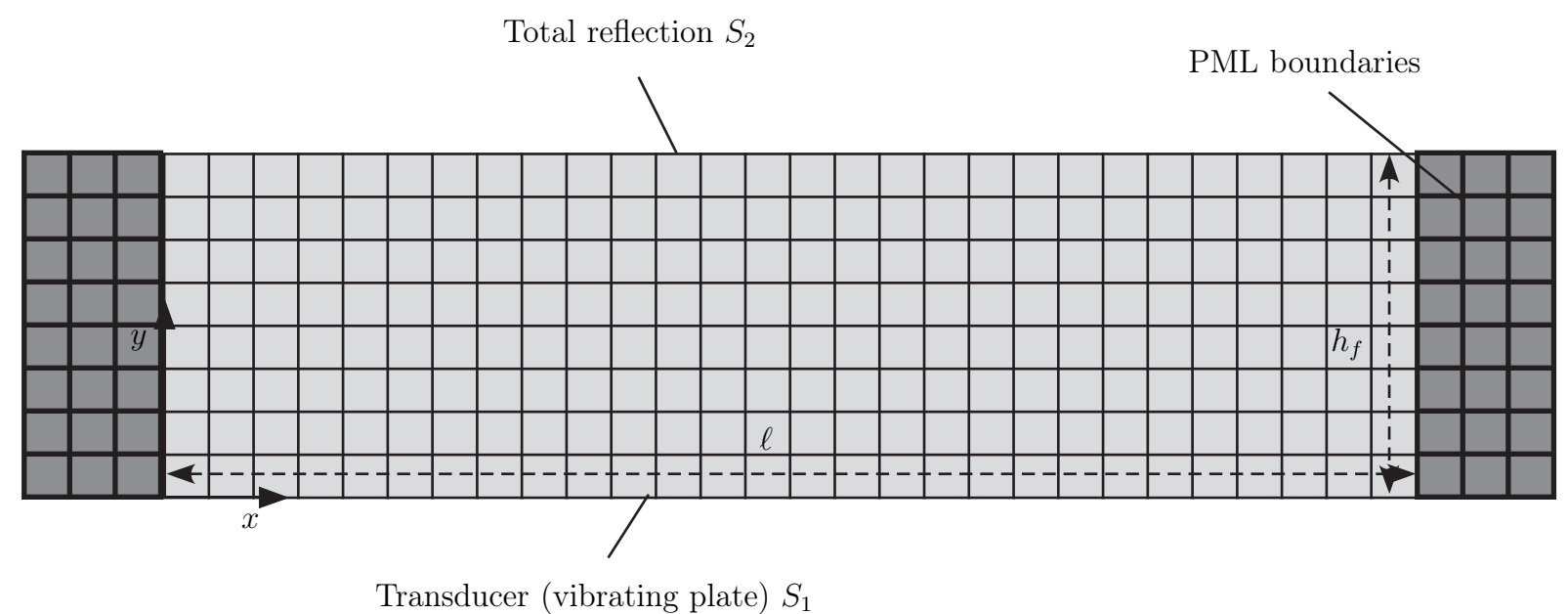

Figure 4.11: Model of the acoustic field with the PML layers.

In the finite element formulation, only the matrix $\mathbf{H}_{\mathbf{e}}$ (eq. 4.26) is affected inside the PML boundaries:

$$
\mathbf{H}_{\mathbf{e}}=\int_{A_{e}}\left(\frac{1}{1+j \frac{\sigma_{x}(x)}{\omega}} \frac{\partial N^{T}}{\partial x} \frac{\partial N}{\partial x}+\frac{1}{1+j \frac{\sigma_{y}(y)}{\omega}} \frac{\partial N^{T}}{\partial y} \frac{\partial N}{\partial y}\right) \mathrm{d} x \mathrm{~d} y
$$

The size of the PMLs is about a wavelength and its contour have the Dirichlet boundary condition $\hat{P}=0$. The reflector is modeled as an unmeshed line where the free Neumann boundary condition $\frac{\partial \hat{P}}{\partial n}=0$ apply.

\section{c) MatLab implementation}

The finite element model developed in the previous section is implemented in the numerical computing software MatLab developed by MathWorks. The full program is detailed in appendix A.1. 


\subsubsection{D model}

A 3D model of the acoustic field will also be needed to determine the width of the prototype. Therefore, an acoustic levitation transportation system model of infinite length and parameterizable width is made by using ANSYS APDL and is presented in fig.4.12.

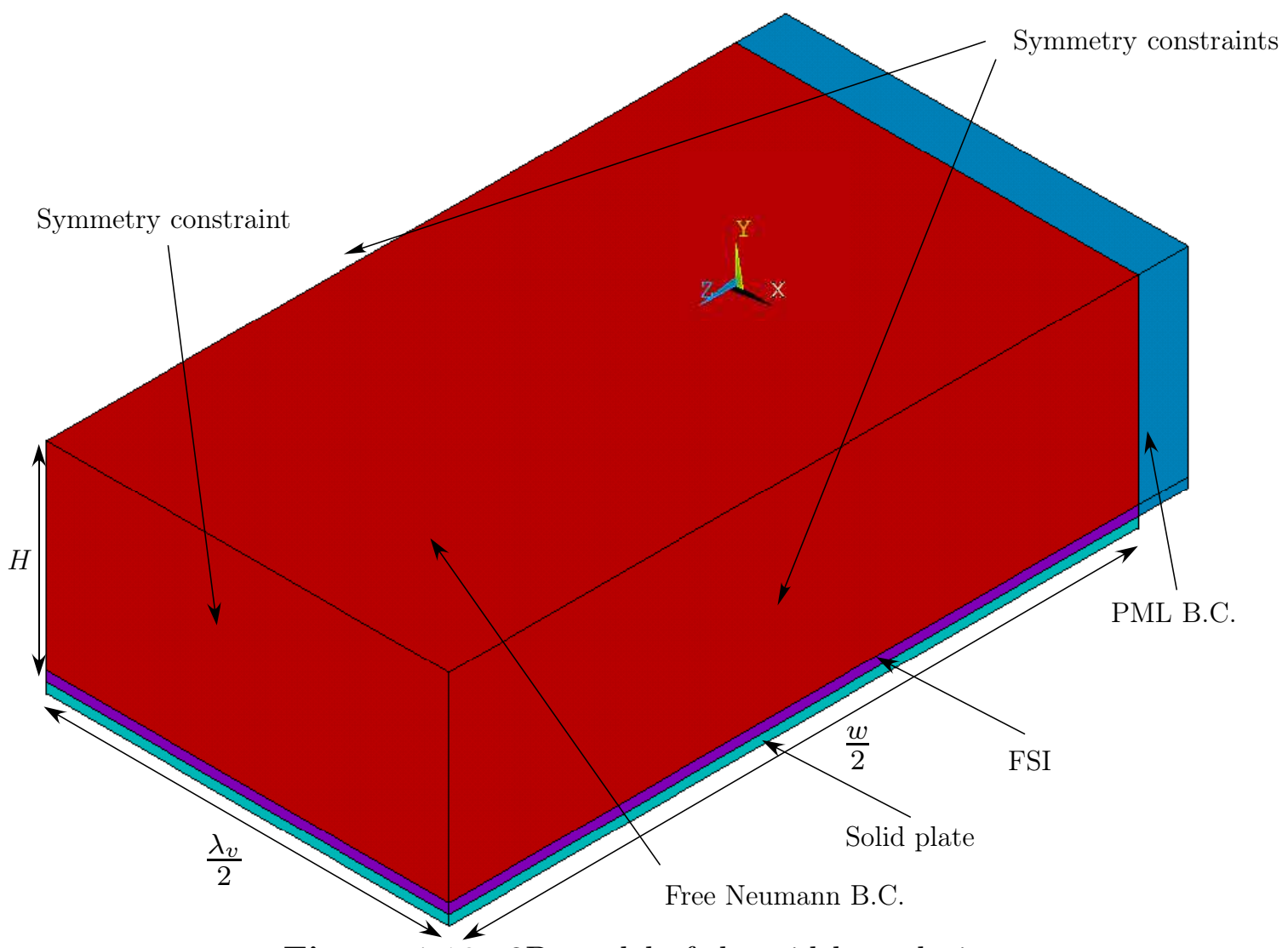

Figure 4.12: $3 \mathrm{D}$ model of the width analysis.

The model consists of a plate of half wavelength of the vibrating plate ${ }^{\lambda_{v}} / 2$ where both sides have an area symmetry boundary condition, half the width of the vibrating plate where one side have an area symmetry boundary condition and the other side is free. The thickness of the plate is one element, the element SOLID185 presented earlier is used, and in order to simulate flexural vibrations every nodes have an imposed displacement boundary condition. The fluid is a brick volume (in red in fig.4.12) has a width and length equal to the plate, a thickness ' $H$ ' and is discretized by using the ANSYS acoustic element FLUID30 (see fig.4.13). Like the plate, both sides of the fluid in the length direction have an area symmetry boundary condition to simulate an infinite length, and in the width direction one side has an area symmetry boundary condition and the other side has a PML boundary condition to simulate a no reflection boundary condition (see $\S$ b)). The top boundary condition of the fluid is set as a free to simulate total wave reflection. The fluid-structural interaction (FSI) is one element thick and placed between the plate and the fluid. The element size of the model is chosen as $H / 20$ as it gives accurate results as 
shown by (FORESTI; BJELOBRK, 2011).

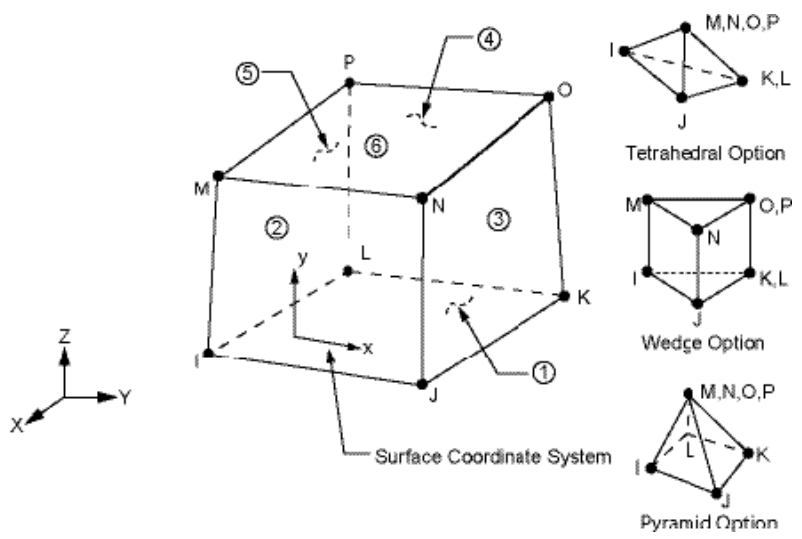

Figure 4.13: ANSYS acoustic element FLUID30.

The FLUID30 element can be used not only to model the acoustic field, but also to define the fluid-structural interaction (FSI) and the perfectly matched layers (PML, see section b)).

\subsection{Finite element iterative design by using ANSYS and MatLab}

In the next chapter, the design of an acoustic levitation transportation system using a ring-type vibrator will be discussed. In order to choose the best design parameters of the prototype, finite element simulations on a wide range of values must be done. ANSYS scripting language APDL allows the use of parameters, and so it can interact easily with MatLab.

First, a template APDL script of the finite element simulation is written, by using variables for the parameters that need iterative analysis, as in our case geometric parameters such as length, width and so on. This template must also include results exported to the post-processing, such as, for example, node pressure/displacement. Those results are exported as tables onto '.txt' files that can be easily imported in MatLab (using the importdata command). Then, a MatLab function can add the variable parameter values inside the APDL template and execute the simulation by using command line (using the system command). Once the simulation is completed, results are imported as arrays or matrices into the MatLab workspace, ready to be treated.

Using the previous MatLab function, iterative design can be easily implemented by using, for example, loops that increments parameters, and therefore plots that show the impact of those parameters can be easily made and analysed. 


\section{Design of the prototype}

\subsection{Introduction}

In this chapter a prototype for an acoustic levitation transportation system using a ring-type vibrator is designed. The objective here is to choose each parameter of the ring-type vibrator to ensure good performance. Since the material, the length and the thickness of the vibrator are pre-defined, the parameters to be designed are:

- The width $w$ of the vibrator;

- The radius $R$ of the vibrator;

- The working frequency $f$;

- The Langevin-type transducers and theirs horns;

As for the reflector, its length and width are always equals to the length and width of the vibrator throughout the design process of the vibrator.

\subsection{Length, thickness and material of the vibrator}

The length $L$ of the vibrator is a free parameter that can be chosen almost arbitrarily as it will define the maximum distance that the levitated particle can travel in a straight line. Here, the length is set as $L=220 \mathrm{~mm}$. The thickness $h$ is also a free parameter that will mostly impact the frequency. Here, $h=3 \mathrm{~mm}$.

The material used for the vibrator and its transducers is aluminium, as it possesses good acoustic properties and is easily machined. Here and for the rest of the design process the aluminium alloy 5052 is used. This alloy has the following properties:

Table 5.1: Aluminium 5052 physical properties.

\begin{tabular}{ccc}
\hline Property & Value & Unit \\
\hline Young's modulus & 72 & $G P a$ \\
\hline Density & 2680 & $\mathrm{~kg} \cdot \mathrm{m}^{-3}$ \\
\hline Poisson's ratio & 0.33 & - \\
\hline
\end{tabular}




\subsection{Width of the vibrator}

The objective here is to determine the impact of the width of the vibrator on the generated acoustic field. The model used here is a plate of parameterizable width ' $w$ ' and infinite length actuating on a fluid comprised between the plate itself and a reflector on the same width and infinite length. The finite element model used here is detailed section 4.5.2.

The plate is made of aluminium 5052 (see table 5.1) and the fluid here is air with properties detailed in table 5.2. An example plot of the pressure distribution inside the fluid is shown fig.5.1.

Table 5.2: Air acoustical properties.

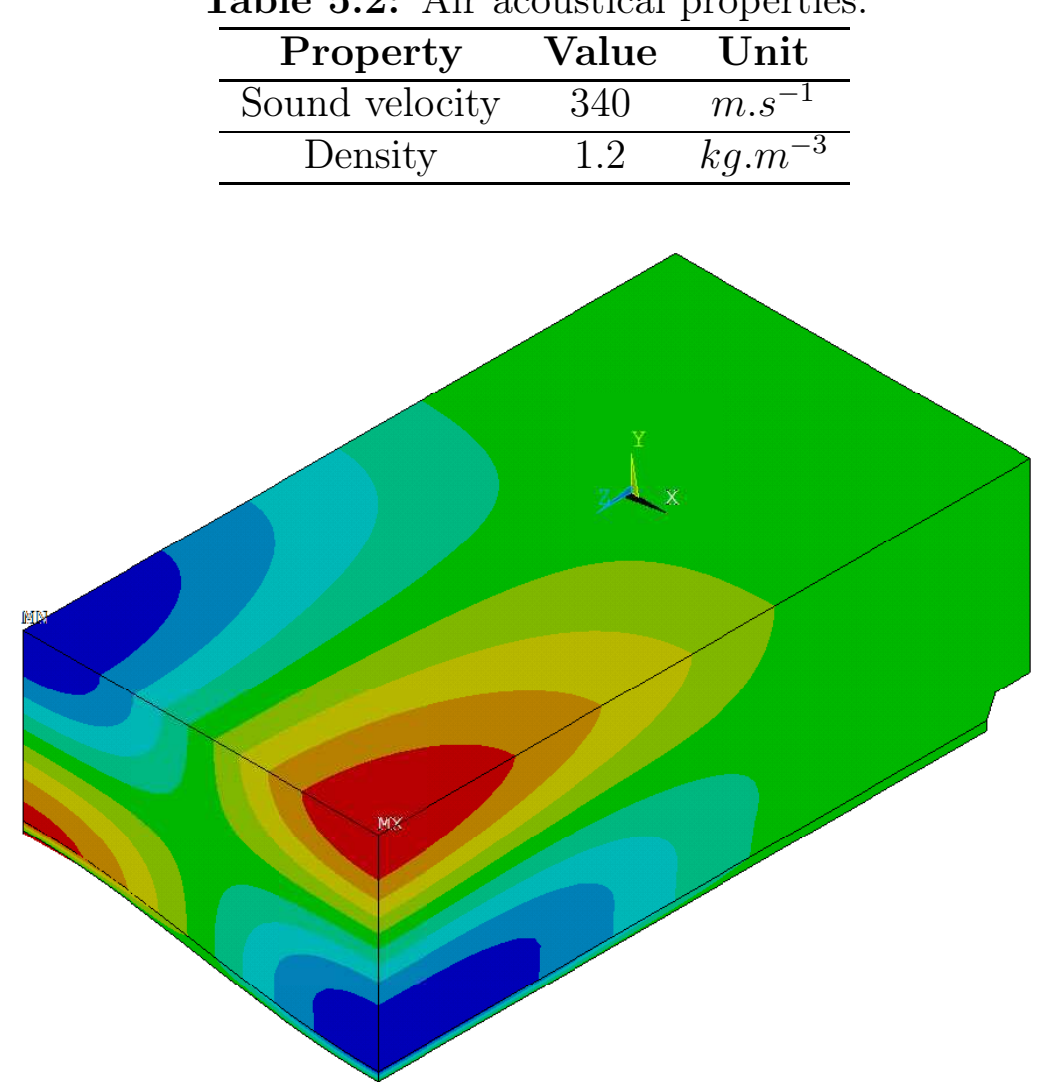

Figure 5.1: Pressure plot of the model.

Plots of the minimum of the dimensionless acoustic levitation potential $\tilde{U}$ (see $\$ 2.2$ ) for various values of the width ' $w$ ' between $30 \mathrm{~mm}$ and $60 \mathrm{~mm}$ are presented in fig.5.2 considering similar parameter of the experiment of (KOYAMA; NAKAMURA, 2010a), i.e. a wavelength inside the vibrating plate of $\lambda_{v}=35 \mathrm{~mm}$ and a frequency of $f=22 \mathrm{kHz}$. Those plots are made by using the iterative design discussed earlier in $\S 4.6$, where the distance between the reflector and the vibrating plate ' $H$ ' is also parameter as the acoustic resonance changes depending on the width and is chosen close to half a wavelength of the acoustic wave $\lambda_{a}=\frac{C_{a i r}}{f}$. As shown in $\S 2.2$, the lower the acoustic levitation potential $\tilde{U}$ is, the higher the forces exerted on the levitated particle are. 


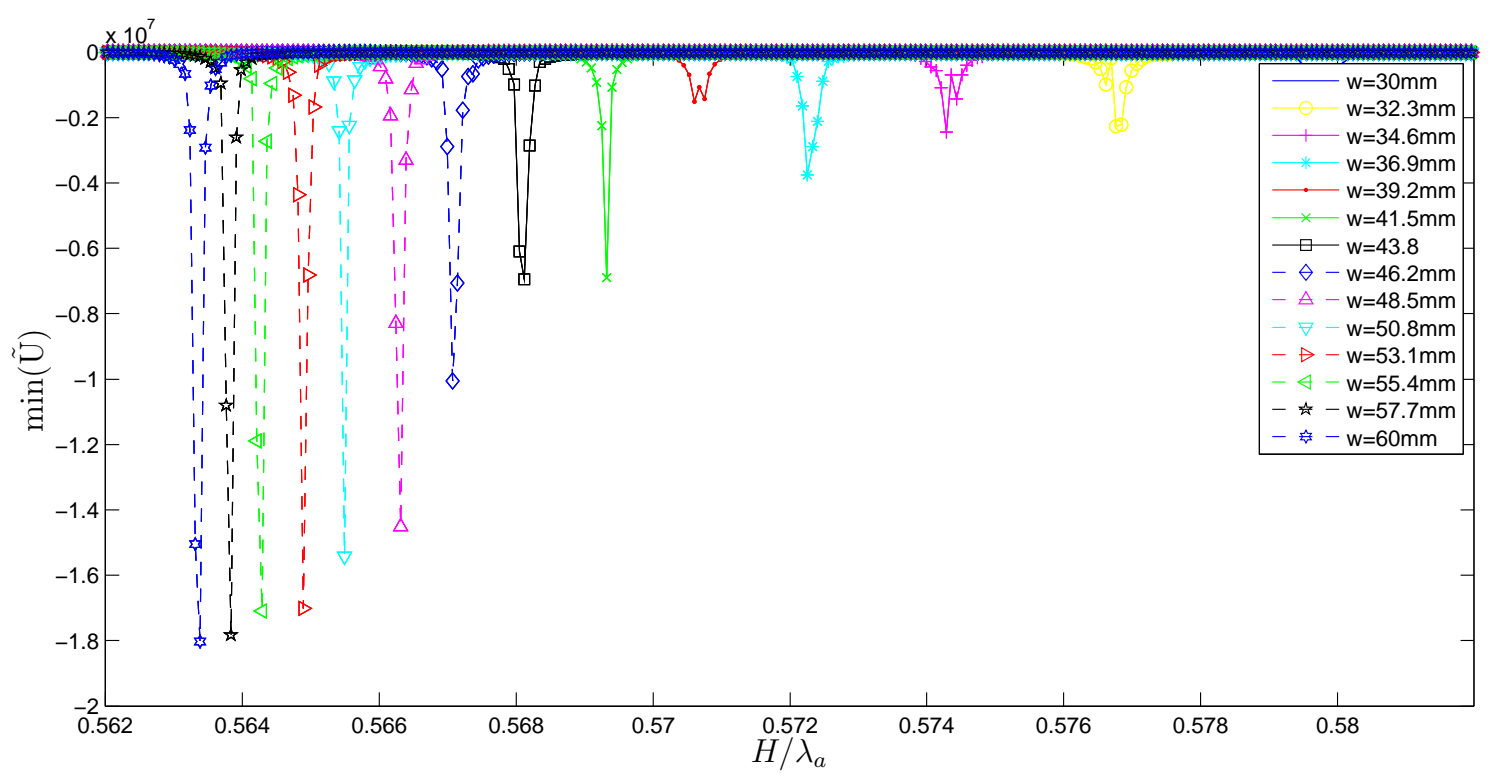

Figure 5.2: Levitation potential minimum as a function of the width of the plate.

From the graph presented in fig. 5.2, we can observe that the wider the vibrating plate the closer the acoustic resonance is to ${ }^{\lambda_{a}} /{ }_{2}$ (or $H / \lambda_{a}=0.5$ ), and from $w=48.5 \mathrm{~mm}$ to $w=60 \mathrm{~mm}$ the difference in acoustic levitation potential minimum is only of $17 \%$.

From the previous analysis, the width of the vibrator is set to $w=50 \mathrm{~mm}$, as a wider plate does not give that much better performance and means more difficulty for the manufacturing of the final prototype.

\subsection{Radius of the vibrator}

The radius of the ring-type vibrator is the most important parameter, as if badly chosen the two pseudo-orthogonal modes needed to allow the translation of the levitated particles will be at separate frequencies, and thus, no travelling wave can be generated. Since the length, thickness and width have been already chosen, the value of the radius will also decide the operating frequency and wavelength of the prototype. In order to evaluate correctly the ring-type vibrator against the straight beam vibrator of (KOYAMA; NAKAMURA, 2010a), the frequency must be chosen close to $22 \mathrm{kHz}$ and the wavelength close to $35 \mathrm{~mm}$.

First, the analysis are made by using the analytical modeling proposed in $\S 3.2$. Plots of the frequency of the two orthogonal modes as a function of the radius $R$ of the ring-type vibrator and the flexural mode $\left(20^{t h}, 22^{n d}\right.$ and $\left.24^{t h}\right)$ are available fig.5.3, 5.4 and 5.5. Since the frequency difference between the two modes is very small, a plot of the frequency difference in percents as a function of the radius is superimposed. The values of the other parameters are the values determined earlier, i.e. $L=220 \mathrm{~mm}, h=3 \mathrm{~mm}, w=50 \mathrm{~mm}$ 
and the material used in the analysis is aluminium 5052 .

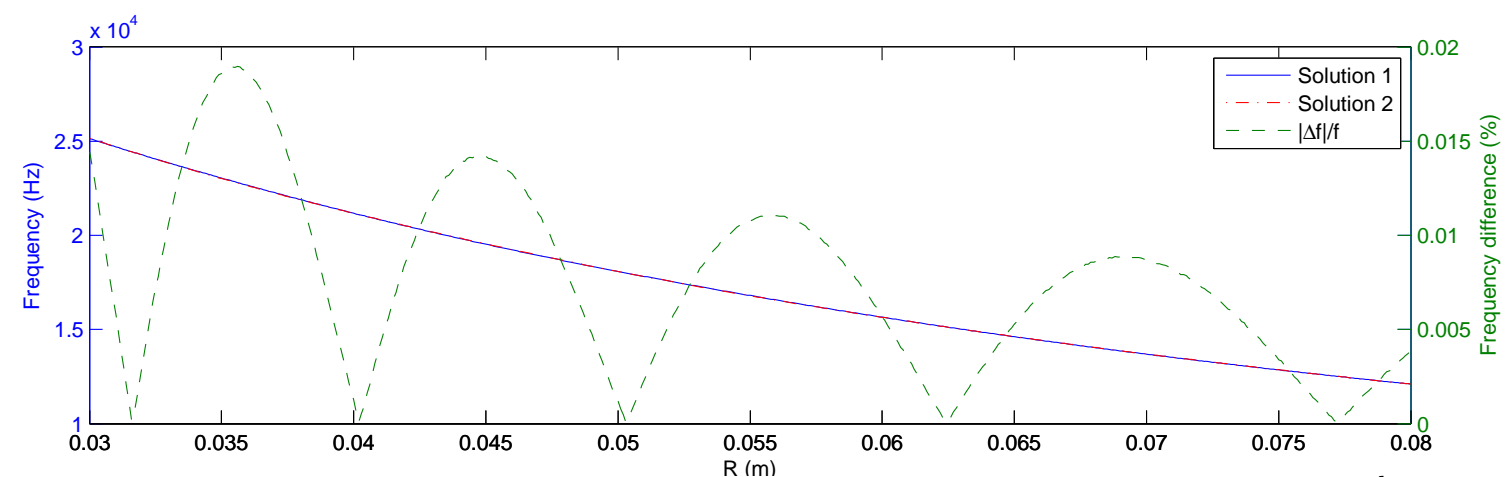

Figure 5.3: Analytical analysis of the frequency of both solutions for the $20^{\text {th }}$ flexural mode.

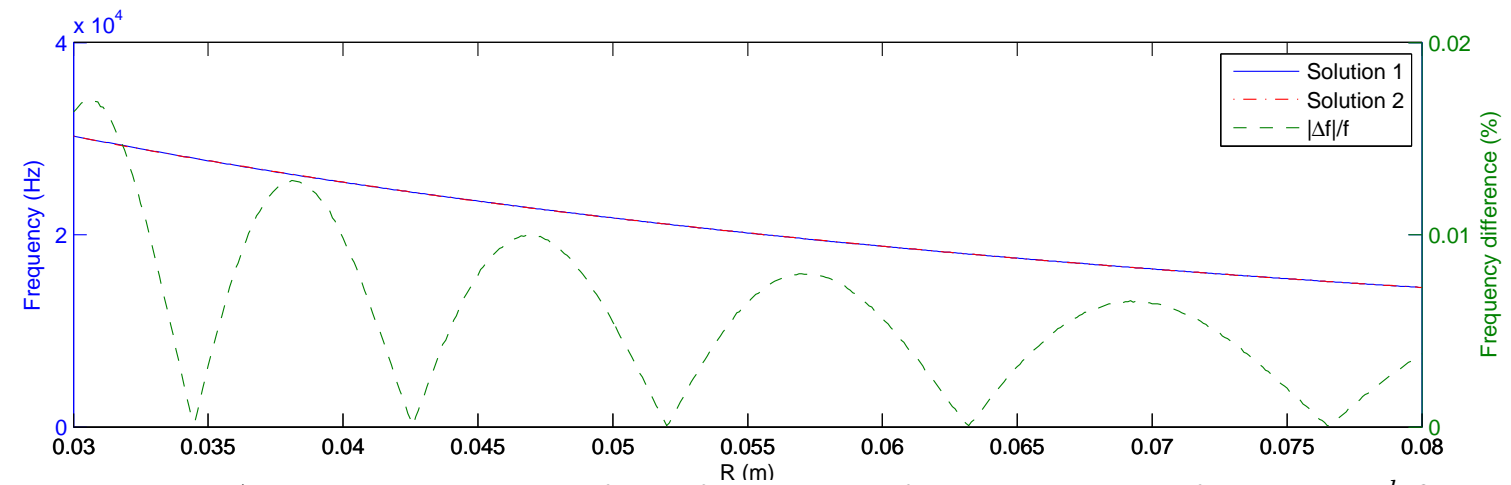

Figure 5.4: Analytical analysis of the frequency of both solutions for the $22^{\text {nd }}$ flexural mode.

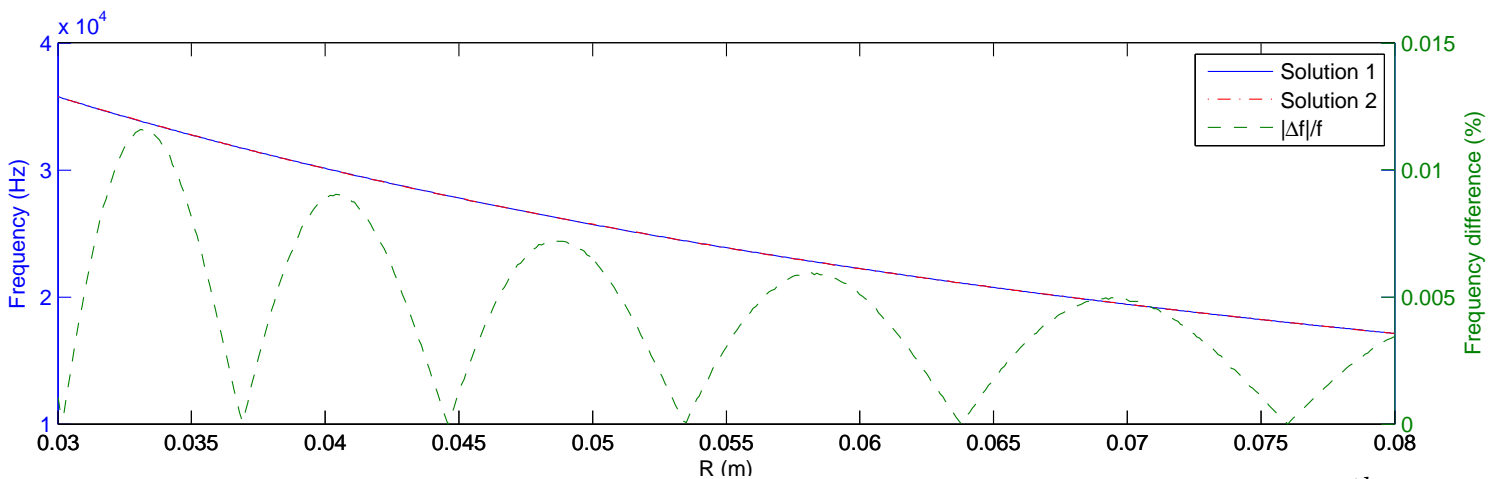

Figure 5.5: Analytical analysis of the frequency of both solutions for the $24^{\text {th }}$ flexural mode.

Each time the frequency difference $\frac{\Delta f}{f}$ is null represent a case where the radius $R$ yield two orthogonal modes at the same frequency, allowing the desired traveling wave. The results of each analysis are summed up in the table 5.3. 
Table 5.3: Results of the analytical analysis.

\begin{tabular}{ccc}
\hline $\mathbf{R}(\mathbf{m m})$ & $\boldsymbol{\lambda}_{\boldsymbol{v}}(\mathbf{m m})$ & $\boldsymbol{f}(\mathbf{H z})$ \\
\hline \hline \multicolumn{3}{c}{$\mathbf{2 0}^{\text {th }}$ flexural mode } \\
\hline \hline 31.6 & 33.8 & 24,438 \\
\hline 40.2 & 36.7 & 21,103 \\
\hline 50.3 & 40 & 18,005 \\
\hline 62.4 & 44 & 15,141 \\
\hline 77.2 & 48.9 & 12,515 \\
\hline \hline \multicolumn{3}{c}{$\mathbf{2 2}^{\text {th }}$ flexural mode } \\
\hline \hline 34.5 & 31.4 & 27,949 \\
\hline 42.6 & 33.9 & 24,415 \\
\hline 52 & 36.7 & 21,106 \\
\hline 63.2 & 40 & 17,993 \\
\hline 76.6 & 44 & 15,124 \\
\hline \hline \multicolumn{3}{c}{$\mathbf{2 4 ^ { \text { th } }}$ flexural mode } \\
\hline \hline 30.2 & 27.5 & 35,635 \\
\hline 36.9 & 29.3 & 31,715 \\
\hline 44.6 & 31.4 & 27,962 \\
\hline 53.5 & 33.9 & 24,407 \\
\hline 63.8 & 36.7 & 21,094 \\
\hline 76.1 & 40 & 17,974 \\
\hline \multicolumn{3}{c}{}
\end{tabular}

Those results are then compared to finite elements analysis, using a complete 3D solid model of the ring type structure (see §4.2) and iterative analysis. The results for the case of the $22^{\text {nd }}$ flexural mode is shown fig.5.6.

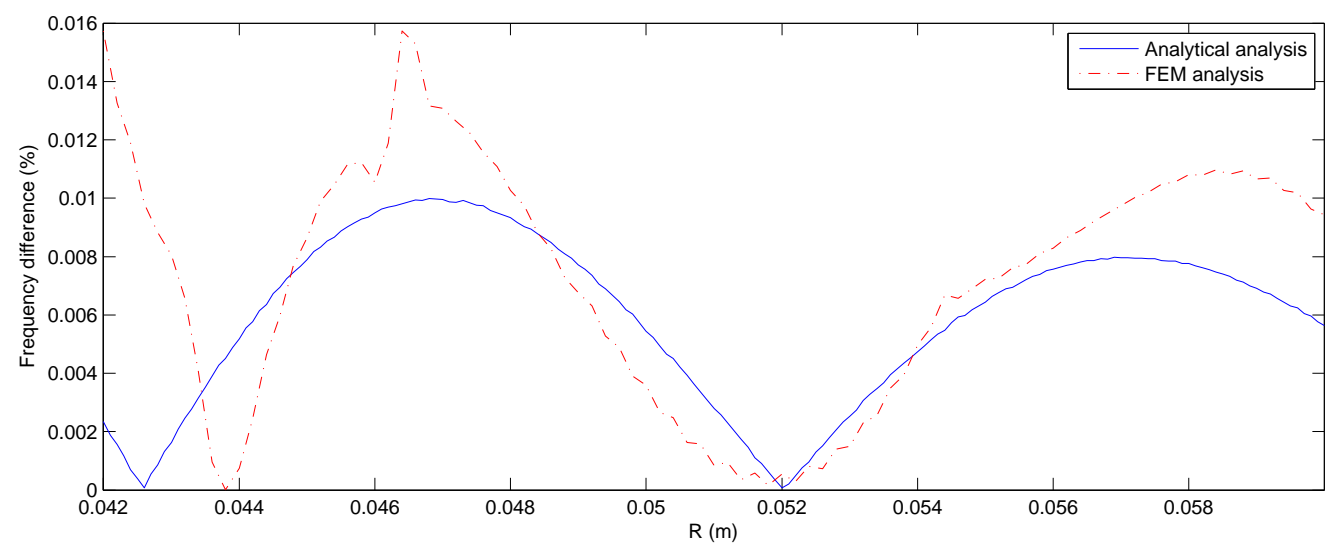

Figure 5.6: Comparison between the analytical analysis and the finite elements analysis for the $22^{\text {nd }}$ flexural mode.

There is a good agreement between the analytical analysis and the finite elements analysis, and for the $22^{\text {nd }}$ flexural mode, we can observe that the frequency difference of the finite elements analysis stay low (inferior to $0.001 \%$ ) between $R=51.5 \mathrm{~mm}$ and $R=52.5 \mathrm{~mm}$, around the analytical result $R=52 \mathrm{~mm}$.

Therefore, the radius $R=52 \mathrm{~mm}$ is chosen for the prototype, giving a working frequency of $f=21,348 \mathrm{~Hz}$ (from the finite elements analysis) and a wavelength for the 
straight part of $\lambda_{v}=36.7 \mathrm{~mm}$.

\subsection{Design of the acoustical horns}

As shown in fig.3.4, the ring-type structure needs to be actuated by two transducers, separated by a distance of $d=\frac{(2 n+1) \lambda_{v}}{4} ; n \in \mathbb{N}$ to allow a spatial phase difference between them of $\pi / 2$. The excitation must be exerted along a line, and since the transducer are circular, acoustical horns are used to amplify and apply linearly the forces generated by the Langevin transducers. Two kind of acoustical horn are evaluated here: the conical profile horn and the stepped profile horn (see §2.3.3). Both horns and their parameters are represented fig. 5.7.

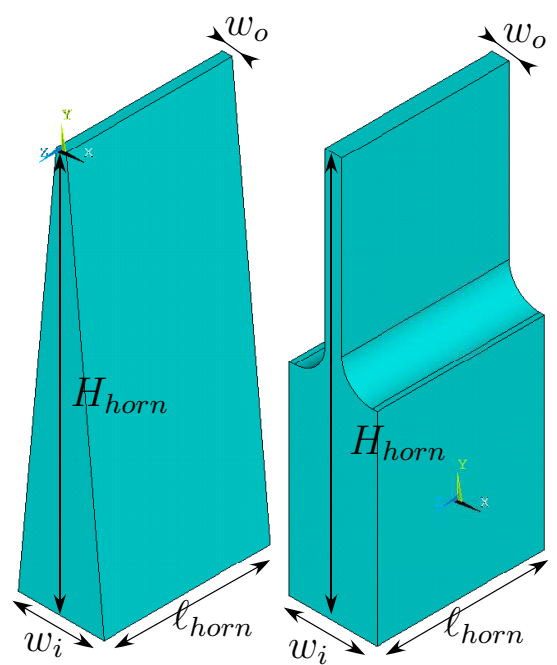

Figure 5.7: Parameters of the acoustical horns (left: conical profile horn; right: stepped profile horn).

The width of the excitation line (and therefore of the output of the acoustical horn) must be as small as possible in order to allow flexural vibrations at the clamping point between the horn and the vibrator, and thus is set as $w_{o}=3 \mathrm{~mm}$, the same as the thickness of the ring-type structure. The width of the input side is set by the Langevin transducer (see next section), and here $w_{i}=26 \mathrm{~mm}$. The thickness of the horn is equal to the width of the vibrator, giving $\ell_{\text {horn }}=w=50 \mathrm{~mm}$.

The length of the horn $H_{\text {horn }}$ is first estimated by calculating the half-wavelength inside the material, i.e. here $\frac{\lambda_{a l}}{2}=\sqrt{\frac{E_{a l}}{\rho_{a l}}} \frac{1}{2 f}=121 \mathrm{~mm}$, and then the length $H_{\text {horn }}$ is adjusted by doing modal analysis until the frequency of the first longitudinal mode is matched with the working frequency of the prototype. The resulting parameters, frequency and amplification factor for both horn are presented in table 5.4. Details for the conical horn are presented in fig.5.8 and table 5.5, and for the stepped horn in fig.5.9 and table 5.6. 
Table 5.4: Parameters of the designed horns.

\begin{tabular}{c|cccc|cc}
\hline Horn profile & $\boldsymbol{w}_{\boldsymbol{o}}$ & $\boldsymbol{w}_{\boldsymbol{i}}$ & $\boldsymbol{\ell}_{\text {horn }}$ & $\boldsymbol{H}_{\text {horn }}$ & $\boldsymbol{f}_{\text {horn }}$ & Amplification \\
\hline Conical & $3 \mathrm{~mm}$ & $26 \mathrm{~mm}$ & $50 \mathrm{~mm}$ & $134 \mathrm{~mm}$ & $21.3 \mathrm{kHz}$ & 2.15 \\
\hline Stepped & $3 \mathrm{~mm}$ & $26 \mathrm{~mm}$ & $50 \mathrm{~mm}$ & $123.8 \mathrm{~mm}$ & $21.3 \mathrm{kHz}$ & 5.04 \\
\hline
\end{tabular}

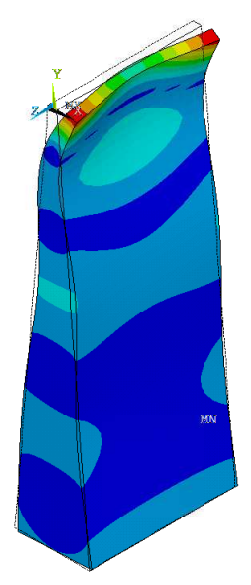

mode a

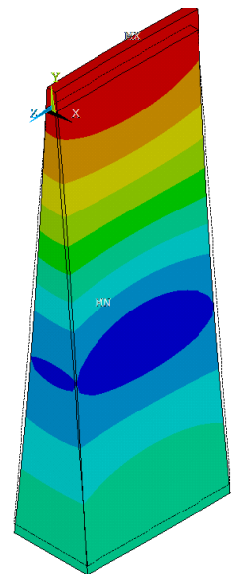

mode $b$

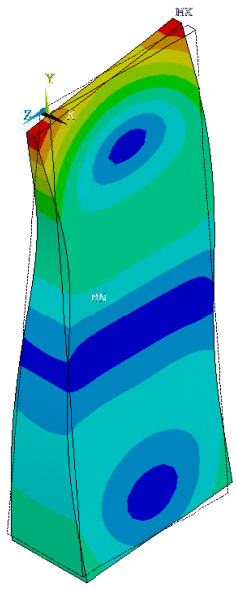

mode $c$

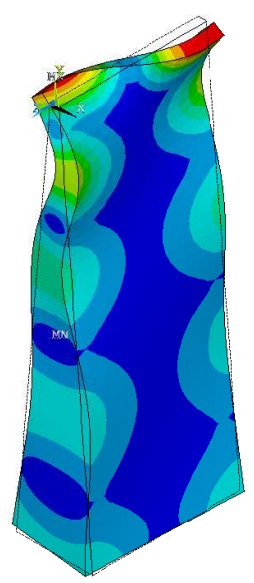

mode $d$

Figure 5.8: The four modes of the conical horn with frequencies close to the working frequency of the prototype.

Table 5.5: Frequencies of the mode of the conical horn close to the working frequency of the prototype.

\begin{tabular}{c|cccc}
\hline & mode a & mode b & mode c & mode d \\
\hline Frequency & $18,117 \mathrm{~Hz}$ & $21,379 \mathrm{~Hz}$ & $22,507 \mathrm{~Hz}$ & $24,884 \mathrm{~Hz}$ \\
\hline
\end{tabular}

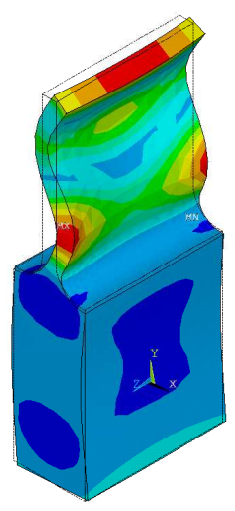

mode a

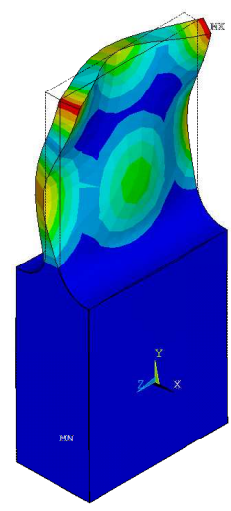

mode $b$

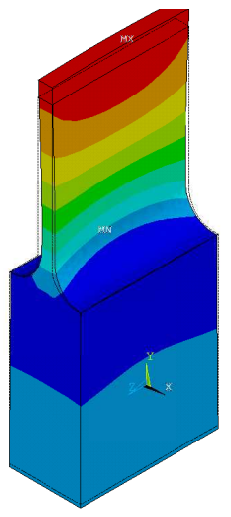

mode $c$

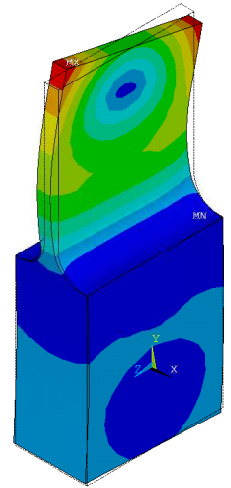

mode d

Figure 5.9: The four modes of the stepped horn with frequencies close to the working frequency of the prototype.

Table 5.6: Frequencies of the mode of the stepped horn close to the working frequency of the prototype.

\begin{tabular}{c|cccc}
\hline & mode a & mode b & mode c & mode d \\
\hline Frequency & $20,195 \mathrm{~Hz}$ & $21,256 \mathrm{~Hz}$ & $21,349 \mathrm{~Hz}$ & $24,151 \mathrm{~Hz}$ \\
\hline
\end{tabular}


In the case of the stepped horn, mode $\mathrm{b}$ and mode $\mathrm{c}$ have very close frequencies $(\Delta f=93 \mathrm{~Hz}$ ), and can be a problem for the experimental part as if the working frequency calculated of the prototype is $100 \mathrm{~Hz}$ off, which is very plausible, the horn will not work with the desire mode and can affect the overall performance of the prototype. Therefore, the conical horn is chosen for the prototype, even if the amplification is far lower than the stepped horn.

As explain in section 3.1, the distance $d$ between the excitation points, and thus between the two horn, must be $d=\frac{(2 n+1) \lambda_{v}}{4} ; n \in \mathbb{N}$ in order to have the $\pi / 2$ spatial phase difference needed to create the traveling wave. Here, $\lambda_{v}=36.7 \mathrm{~mm}$, as set in the section about the radius of the vibrator. It is hard to evaluate the impact of the position of the horns on the amplitude of the standing wave in the vibrator, since the structural damping is unknown and must be chosen arbitrarily, however it is almost certain that the amplitude of the standing wave in the section between the horns will be higher than on the other side, and thus the distance $d$ must be chosen as high as possible in order to have a good repartition of the amplitude. Also, the horns are the only ties of the ring-type vibrator, and thus, in order to obtain a good structural stability the distance between the horns must be high. Therefore, $d$ is chosen as $d=192.68 \mathrm{~mm}$ (i.e. $n=10$ ).

\subsection{Design of the transducers}

The excitation is realized by using Langevin-type transducer, detailed in section 2.3, coupled with mechanical horns in order to have high displacements. Since the working frequency of the prototype is calculated as $f=21,348 \mathrm{~Hz}$, both transducer must have their resonant frequency equal to $f$. In order to optimise to the maximum the impedance matching between the ring-type vibrator and the transducers, the material chosen is the same aluminium alloy 5052 detailed in table 5.1. The piezoelectric ceramic rings used here are made of PZT4 which properties are detailed in table 5.7, and have an outer diameter of $1.5^{\prime \prime}(38.1 \mathrm{~mm})$, an inner diameter of $0.5^{\prime \prime}(12.7 \mathrm{~mm})$ and a thickness of 0.25 " $(6.35 \mathrm{~mm})$. Those ceramics are chosen arbitrarily as they are already available in large number at the laboratory and their properties are verified, thus making the design easier and more accurate.

First, modal analysis are made on the transducer and the mechanical amplifier separately, and then harmonic analysis are made on the coupled model in order to tune the parameters. The chosen parameters of the transducers are resumed table 5.8 and correspond to the parameters set by (ABDULLAH; SHAHINI; PAK, 2009) in fig.2.2 (see section 2.3) 
Table 5.7: PZT4 properties.

\begin{tabular}{cccc}
\hline Property & Symbol & Value & Unit \\
\hline Density & $\rho_{p z t}$ & 7500 & $\mathrm{~kg} \cdot \mathrm{m}^{-3}$ \\
\hline \multirow{2}{*}{ Piezoelectric coupling coefficients } & $d_{31}$ & $-1.2310^{-10}$ & \\
& $d_{33}$ & $2.8910^{-10}$ & $\mathrm{~m} . \mathrm{V}^{-1}$ \\
& $d_{15}$ & $4.9610^{-10}$ & \\
\hline & $s_{11}^{E}$ & $1.2310^{-11}$ & \\
& $s_{33}^{E}$ & $1.5510^{-11}$ & \\
Compliance & $s_{12}^{E}$ & $-4.0510^{-12}$ & \\
& $s_{13}^{E}$ & $-5.3110^{-12}$ & $\mathrm{~m} . \mathrm{s}^{2} . \mathrm{kg}^{-1}$ \\
& $s_{44}^{E}$ & $3.9010^{-11}$ & \\
& $s_{66}^{E}$ & - & \\
\hline \multirow{2}{*}{ Electric permittivity } & $\varepsilon_{11}^{T}$ & $1.3110^{-8}$ & \multirow{2}{*}{$\mathrm{F} \cdot \mathrm{m}^{-1}$} \\
& $\varepsilon_{33}^{T}$ & $1.1510^{-8}$ & \\
\hline
\end{tabular}

Table 5.8: Parameters of the designed transducer.

\begin{tabular}{ccc}
\hline Symbol & Value & Unit \\
\hline$D_{B}$ & 40 & $\mathrm{~mm}$ \\
\hline$D_{F 2}$ & 26 & $\mathrm{~mm}$ \\
\hline$H_{B}$ & 49 & $\mathrm{~mm}$ \\
\hline$H_{B 2}$ & 8 & $\mathrm{~mm}$ \\
\hline$N_{c}$ & 2 & - \\
\hline$H_{F}$ & 162.8 & $\mathrm{~mm}$ \\
\hline$H_{F 1}$ & 105.9 & $\mathrm{~mm}$
\end{tabular}

By using the empirical recommendations of (ABDULLAH; SHAHINI; PAK, 2009) for a transducer of $20 \mathrm{kHz}$ and $1 \mathrm{~kW}$, the central bolt used is a M10 steel, with a length $L_{B t}=80 \mathrm{~mm}$, and the torque is set as $100 \mathrm{~N} . \mathrm{m}$. 


\section{Analysis}

\subsection{Beam vibrator prototype}

This chapter will focus on reproducing, with the preceding modeling, the results obtained by D. Koyama and K. Nakamura in their paper (KOYAMA; NAKAMURA, 2010a). The system described in the paper consists of a $3 \mathrm{~mm}$ thick, $605 \mathrm{~mm}$ long and $60 \mathrm{~mm}$ wide duraluminium vibrating plate and a plate with the same dimension used as a reflector, as presented in fig. 1.4. The reflector is installed parallel to the vibrating plate at a distance of approximately $17 \mathrm{~mm}$, and the vibrating plate is actuated by two Langevin transducer at $35 \mathrm{~mm}$ from the plate edges, generating a flexural wave along the vibrating plate at $22.5 \mathrm{kHz}$. The flexural wave generated can be translated by changing the driving phase difference between the two Langevin transducer. The experimental setup successfully levitated and moved polystyrene sphere of few millimeters diameter along $60 \mathrm{~mm}$ with an accuracy of $0.046 \mathrm{~mm} / \mathrm{deg}(2.636 \mathrm{~mm} / \mathrm{rad})$.

\subsubsection{Analysis of the vibrating plate}

The properties of the duraluminium vibrating plate used in the original paper are presented in table 6.1 .

Table 6.1: Properties of the vibrating plate designed in (KOYAMA; NAKAMURA, 2010a)

\begin{tabular}{ccc}
\hline Property & Value & Unit \\
\hline Length & 0.605 & $\mathrm{~m}$ \\
Width & 0.06 & $\mathrm{~m}$ \\
Thickness & 0.003 & $\mathrm{~m}$ \\
\hline Young's modulus & 73 & $\mathrm{GPa}$ \\
Density & 2800 & $\mathrm{~kg} \cdot \mathrm{m}^{-3}$ \\
\hline
\end{tabular}

\section{a) Modal analysis}

First, a modal analysis of the vibrating plate is made in order to find the frequency of each flexural mode. Since a flexural mode at $22.5 \mathrm{kHz}$ and a wavelength of approximately 
$35 \mathrm{~mm}$ is used in the experiments of the original paper, the analysis is made around this frequency and is summed up in the table 6.2. The number of elements used in this analysis is 800, which give more than 10 elements per bending as discussed previously in A.1.3.

Table 6.2: Modal analysis of the vibrating plate designed in (KOYAMA; NAKAMURA, 2010a).

\begin{tabular}{ccc}
\hline Flexural mode & Frequency $(\mathbf{k H z})$ & Wavelength $\mathbf{( m m})$ \\
\hline $30^{t h}$ & 17.65 & 40.1 \\
$31^{\text {st }}$ & 18.83 & 38.6 \\
$32^{\text {nd }}$ & 20.04 & 37.1 \\
$33^{\text {rd }}$ & 21.30 & 36.3 \\
$34^{\text {th }}$ & 22.59 & 34.8 \\
$35^{\text {th }}$ & 23.92 & 34.1 \\
$36^{\text {th }}$ & 25.28 & 32.5 \\
\hline
\end{tabular}

The mode closer to the mode used in the paper is the $34^{\text {th }}$ flexural mode, with a natural frequency of $22.59 \mathrm{kHz}$ and a wavelength of $34.8 \mathrm{~mm}$, although the authors mention another mode using a larger wavelength, probably the $33^{\text {rd }}$.

\section{b) Harmonic analysis}

The harmonic analysis of the vibrating plate will give the response of the plate to the excitation induced by the two driving transducers (named $\hat{F}_{1}=F_{1} e^{j \varphi_{1}}$ and $\hat{F}_{2}=F_{2} e^{j \varphi_{2}}$ in the modeling).

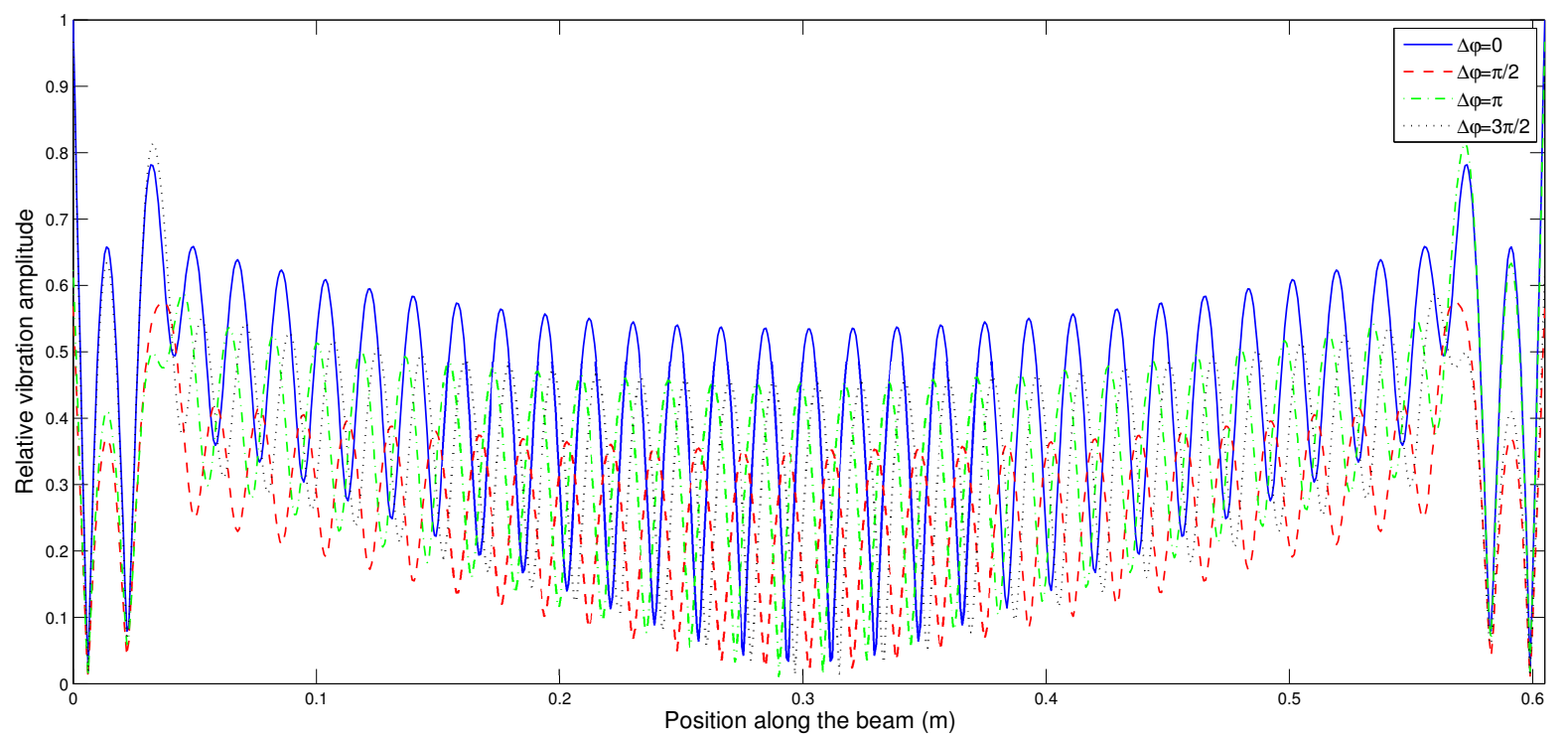

Figure 6.1: Relative amplitude of vibration along the beam for some driving phase difference.

Harmonic analysis are made for some driving phases in fig. 6.1. Those harmonic analysis permit the following observations: 
- The amplitudes decrease until a driving phase difference equal to $\pi$ and increases thereafter.

- The nodes of vibration do not reach a zero amplitude except in the middle and ends of the beam.

- The nodes and anti-nodes of vibration are translated between the position where $\hat{F}_{1}$ and $\hat{F}_{2}$ are applied, however not beyond.

The fact that the nodes of vibration, independently of the driving phase difference, do not reach a zero amplitude means that there is a traveling wave added to the standing wave. For a fixed driving phase difference, the vibration along the beam for a period of time $T=\frac{2 \pi}{\omega}$ is represented fig. 6.2 , using equation 6.1 .

$$
v(x, t)=|V(x)| \sin \left(\frac{2 \pi}{T} t-\phi\right)
$$

where $t$ is the time, $v$ is the transverse deflection of the beam, and $|V|$ and $\phi$ are the complex modulus and phase of the transverse deflection calculated in the harmonic analysis. This figure reveals that the wave propagates from the middle of the beam to the position of the two actuators, and as also seen in fig. 6.1, the amplitude of the traveling wave increases linearly whereas the amplitude of the standing wave decreases linearly. This traveling wave is undesired and may have an effect on the acoustic field, this point will be discussed later.

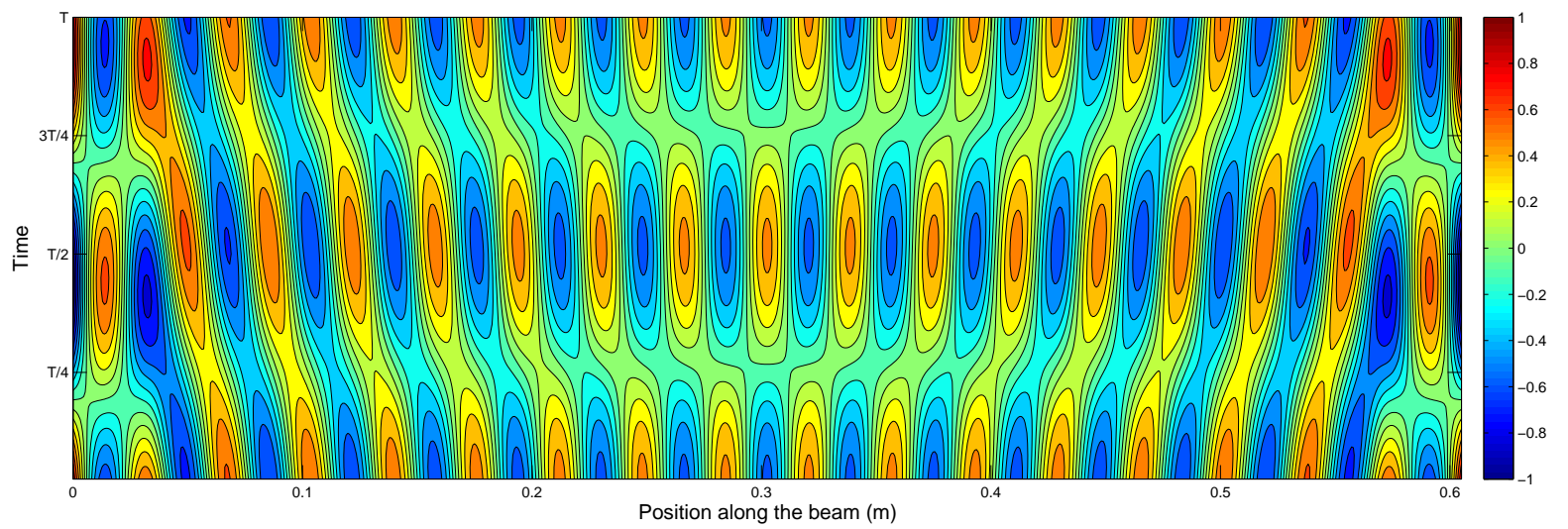

Figure 6.2: Visualization of the vibration along the plate for a complete period of time and $\Delta \varphi=0$.

Changing the phase difference $\Delta \varphi$ between the two actuators $\hat{F}_{1}$ and $\hat{F}_{2}$ effectively translates the standing wave, as reported in the original paper. Figure 6.3 clearly shows that when $\Delta \varphi$ goes from 0 to $2 \pi$, the standing wave is translated of half its wavelength, however only between the two actuators. The direction of the translation can be inverted by simply changing the sign of $\Delta \varphi$. 


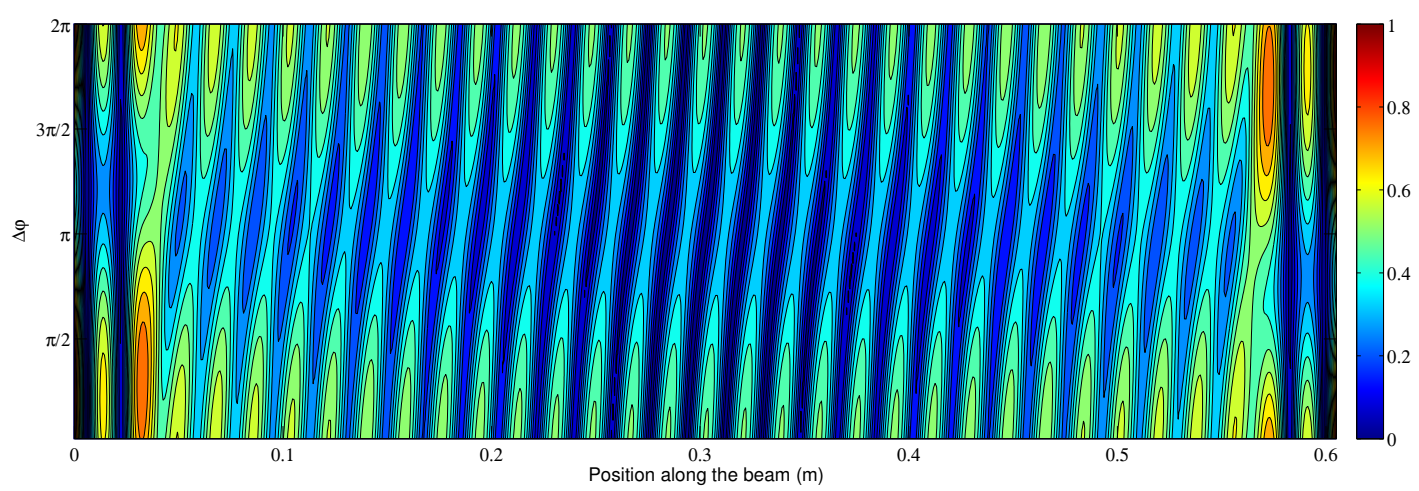

Figure 6.3: Visualization of the vibration amplitude along the plate for $\varphi_{1}=0$ and

$$
\varphi_{2}=\Delta \varphi \text {. }
$$

Figure 6.4 shows the translation realized by the standing wave in function of the driving phase difference. This plot is similar to the experimental plot of the original paper (fig. 9, (KOYAMA; NAKAMURA, 2010a)), except for $\Delta \varphi \in\left[\frac{3 \pi}{4}, \frac{5 \pi}{4}\right]$, where in the experimental plot the moving distance is almost constant whereas in the FEM plot is linear. This can be explain by the fact that for this interval the vibration amplitude is at its minimum (see fig. 6.1), and the measurement may be more difficult and less accurate. This hypothesis is reinforced by the fact that the difference between the moving distance at $\frac{3 \pi}{4}$ and $\frac{5 \pi}{4}$ is almost the same in both plots.

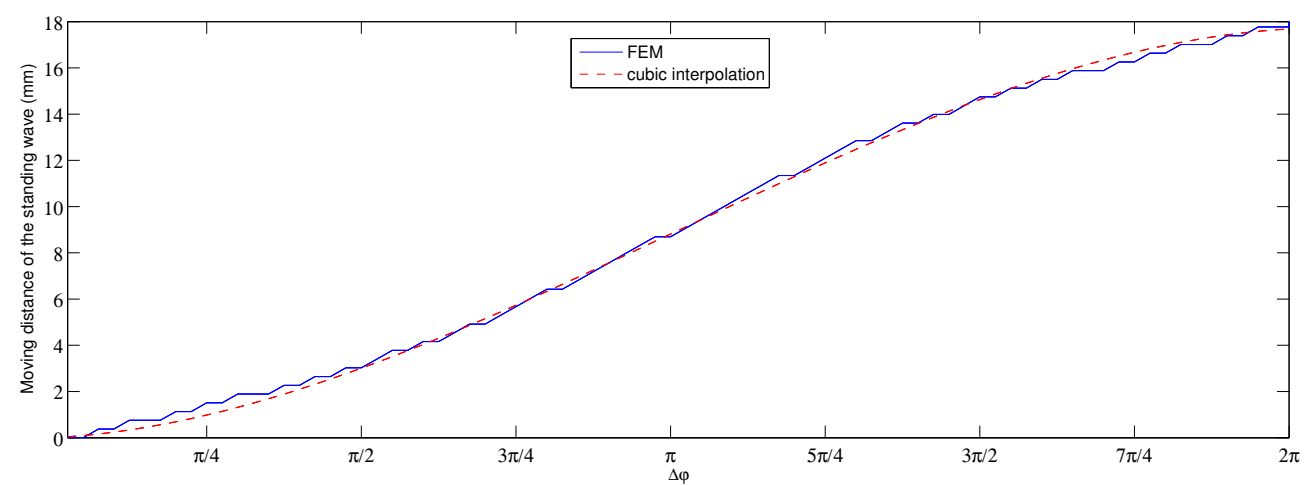

Figure 6.4: Translation of the standing wave in function of the driving phase difference

A relation between the moving distance $\Delta x$ of the standing wave and the driving phase difference can be found by performing a cubic interpolation of the results given by the finite element analysis. The resulting equation is :

$$
\Delta x=a_{1} \Delta \varphi+a_{2} \Delta \varphi^{2}+a_{3} \Delta \varphi^{3}
$$

where the polynomial parameters are: 


$$
\begin{gathered}
a_{1}=5.984 \cdot 10^{-5} \mathrm{~m} \\
a_{2}=1.101 \cdot 10^{-3} \mathrm{~m} \\
a_{3}=-1.195 \cdot 10^{-4} \mathrm{~m}
\end{gathered}
$$

This relation can be useful for implementing a speed and position control of the levitated particle.

\subsubsection{Analysis of the acoustic field}

Once the reflector placed parallel and at an adequate distance of the vibrating plate, an acoustic standing wave can be generated and thus, small radius particles levitated. This distance, is, as shown in the parametric study of acoustic levitator by (XIE; WEI, 2001), not exactly equal to $\lambda_{a}=\frac{c}{f}$ but slightly superior, depending on the geometry of the levitator, and in that case $18.1 \mathrm{~mm}\left(1.9 \lambda_{a}\right)$. The domain discretization is 120 elements in the vertical direction and 800 elements in the horizontal direction. The dimensionless Gor'kov acoustic levitation potential $\tilde{U}$ defined in eq. 2.5 is used to see the possible positions of stable levitation of a rigid particle $\left(f_{1}=f_{2}=1\right)$. A plot showing the pressure field, particle velocity field and the levitation potential around the center of the beam is shown fig. 6.5. Since the acoustic forces are defined as $F=-\nabla U$ (eq. 2.4), a particle can be levitated in a potential well (a local minimum in the levitation potential field).

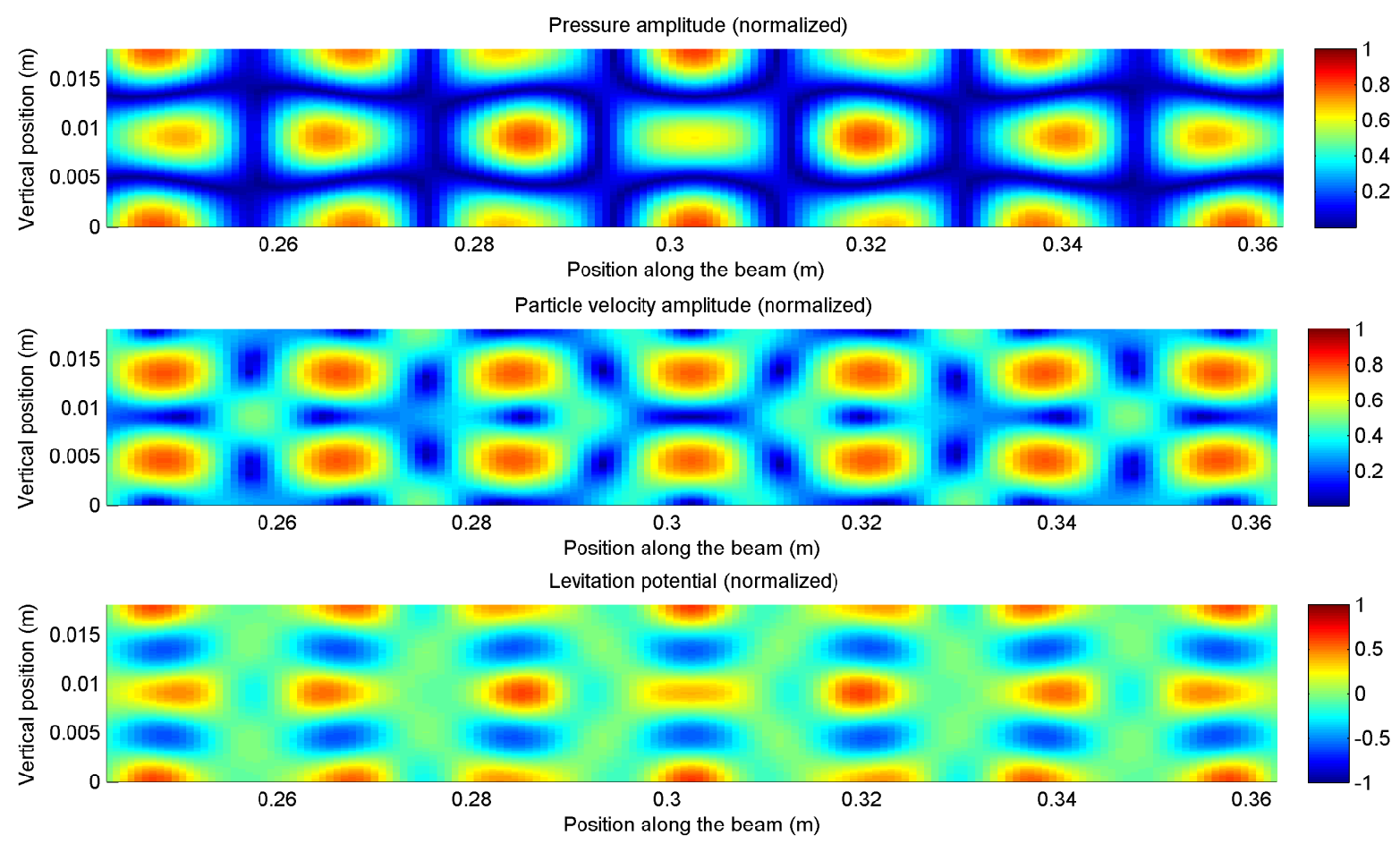

Figure 6.5: Normalized pressure amplitude, velocity amplitude and dimensionless Gor'kov acoustic levitation potential for $\Delta \varphi=0$. 
A comparison between the acoustic field and the vibration amplitude of the vibrating plate shows that the horizontal positions of the Gor'kov levitation wells coincide with the vibration nodes of the vibrating plate. The vertical positions of the potential wells correspond to the positions of the pressure nodes. A wider plot of the Gor'kov acoustic levitation at both extremities is presented in fig. 6.6.
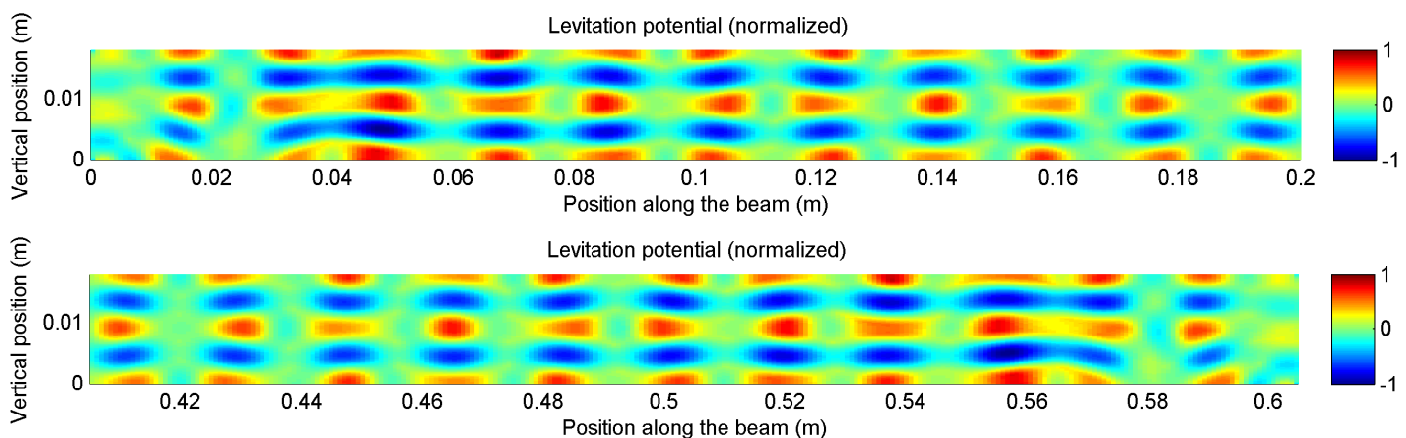

Figure 6.6: Normalized dimensionless Gor'kov acoustic levitation potential for $\Delta \varphi=0$ at the extremities.

This plot shows that the closer to the excitation point of the beam, the less stable the levitation will be, as the potential wells expend until almost merging. This phenomenon can be linked to the time-dependent traveling wave of the vibrating plate which amplitude linearly increases from the middle of the beam to the excitation point (see fig. 6.2 and 6.1). The figure 6.7 presents plots of the levitation potential for 8 values of $\Delta \varphi \in[0,2 \pi]$.

This figure shows that the transport of particles by acoustic levitation is feasible with this system, as the levitation potential wells are displaced by changing the driving phase between the two actuators. Also, the distance traveled is bound to the same equation as the vibration of the beam, i.e. eq. 6.2. 


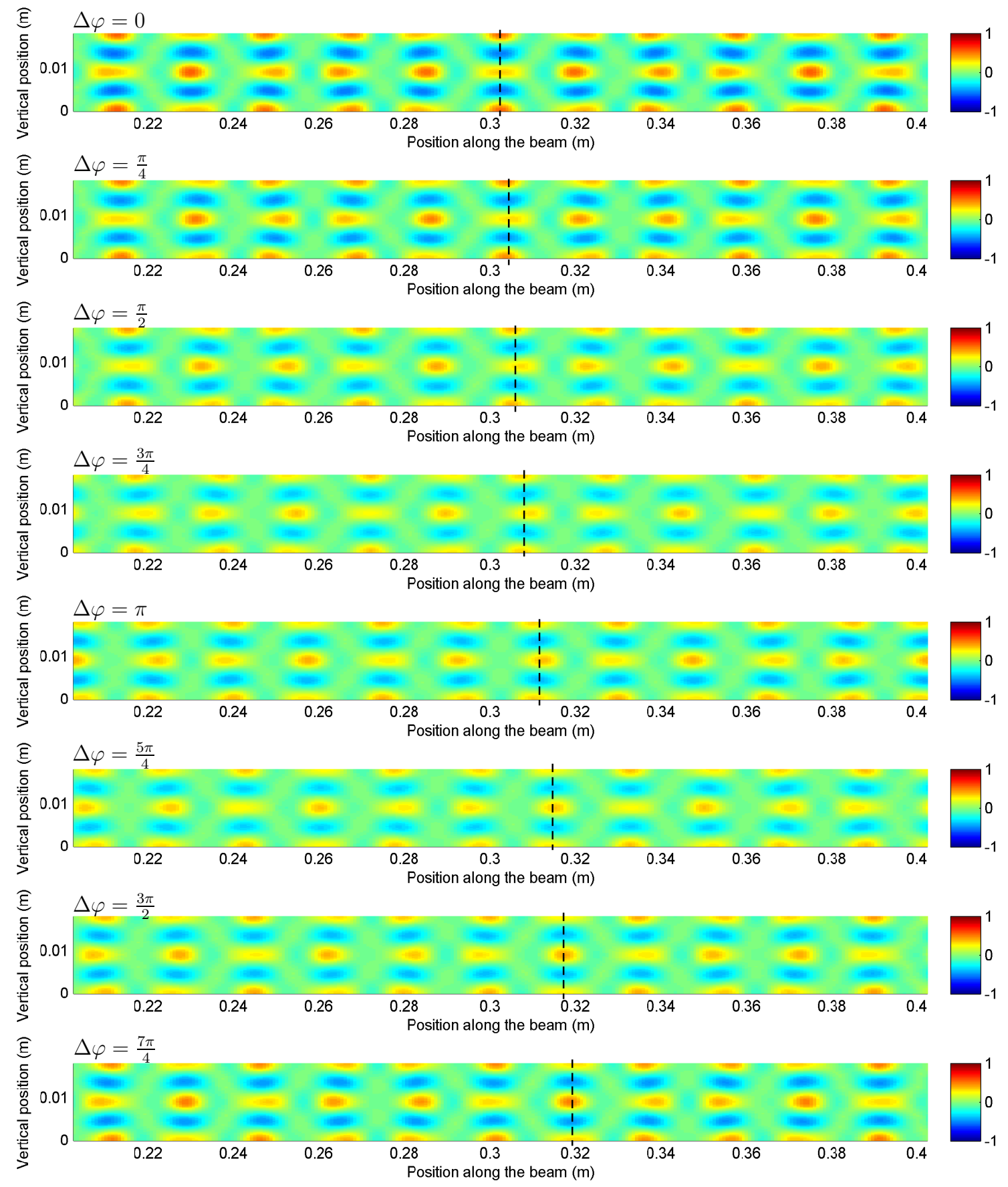

Figure 6.7: Relative dimensionless Gor'kov acoustic levitation potential for $\Delta \varphi \in[0,2 \pi]$. 
Therefore, the model made in chapter 4 is compared with experimental results of the paper (KOYAMA; NAKAMURA, 2010a) and showed good agreement. The model successfully shows the traveling wave in both the plate and the acoustic field, the same way that presented in the referenced paper. Therefore, the model presented here can be used to design other acoustic levitation transportation system of the same kind.

\subsection{Ring-type vibrator prototype}

Once all the parameters of the ring-type vibrator are set in chapter 5, the prototype can be manufactured. The objective of this section is to analyse the behavior of the vibrator and the resulting acoustic field by using the previous models in order to compare those results with the prototype discussed in section 6.1 and later with the experimental results.

\subsubsection{Analysis of the ring-type vibrator}

The ring-type vibrator is designed to have two orthogonal modes at the same frequency using only modal analysis. By doing various harmonic analysis, the traveling wave can be visualized and analysed. For those analysis, the excitation used is a force applied on a line,

along the width of the vibrator, at the positions $x=-\frac{d}{2}$ and $x=\frac{d}{2}$ (see fig.3.4). The force amplitude is chosen as $A=1 N$ as every results will be normalized, and the objective is to predict the spatial phase of the standing wave and observe how its amplitude fluctuates in relation to the variable $\theta$ (in a similar way that the analysis of part 1 ). Since those forces are in temporal phase $\left(f_{1}(\theta, t)=A \cos (\theta) \cos (\omega t)\right.$ and $f_{2}(\theta, t)=A \sin (\theta) \cos (\omega t)$, see section 3.1), the input for the harmonic analysis are $F_{1}=\cos (\theta)$ and $F_{2}=\sin (\theta)$. MatLab and ANSYS are used here in the same way of the iterative design, that is, to process various harmonic analysis and change the value of $\theta$ each time.

Figures 6.8 and 6.9 show the normalized relative vibration amplitudes along both straight part of the vibrator. Figures 6.10 and 6.11 show the actual translation of the standing wave in relation to $\theta$, thus, in relation of the amplitude of the transducers. The part of the ring-type vibrator tied to the horns is referred as the bottom side and the other part as the top side, as detailed later in fig. 8.1. 


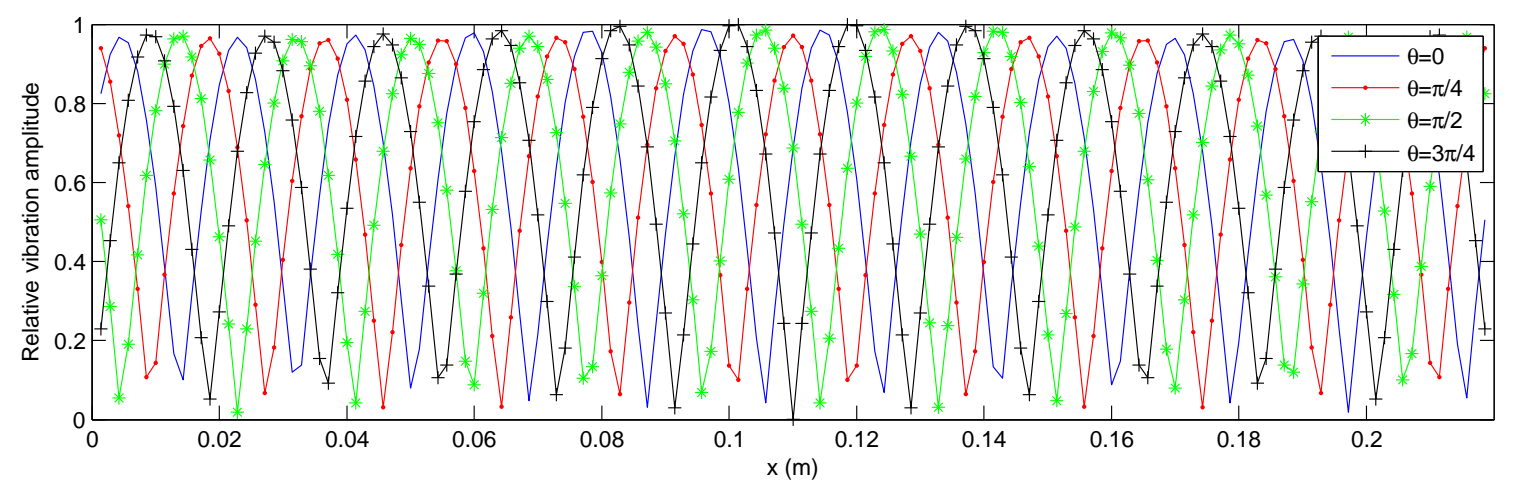

Figure 6.8: Relative vibration amplitude along the top straight part of the vibrator.

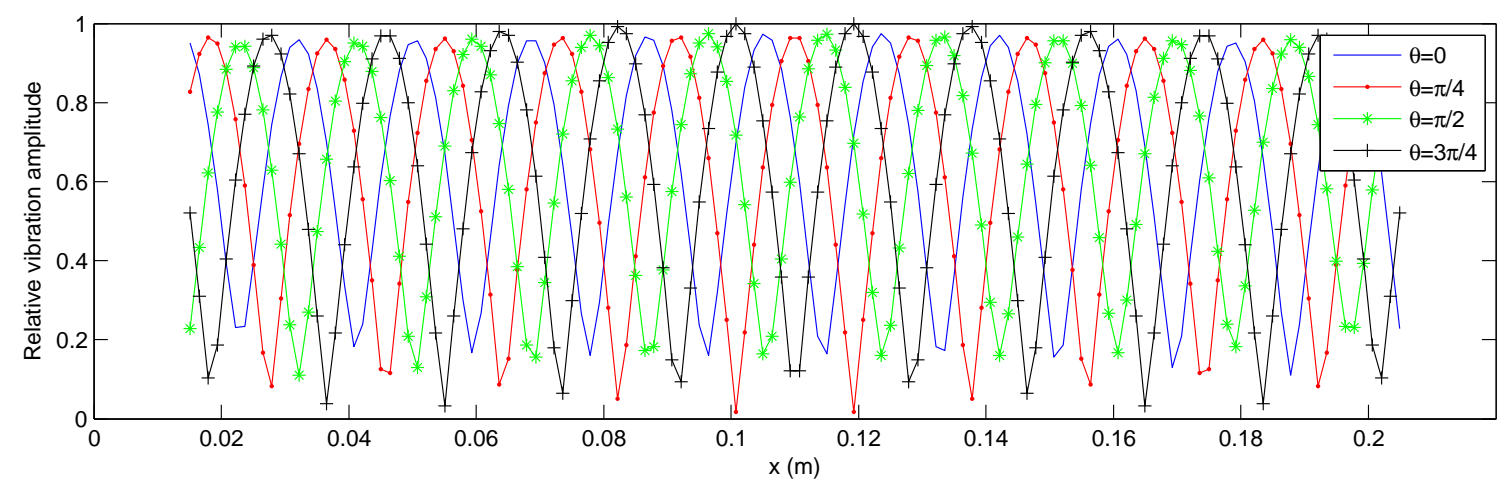

Figure 6.9: Relative vibration amplitude along the bottom straight part of the vibrator.

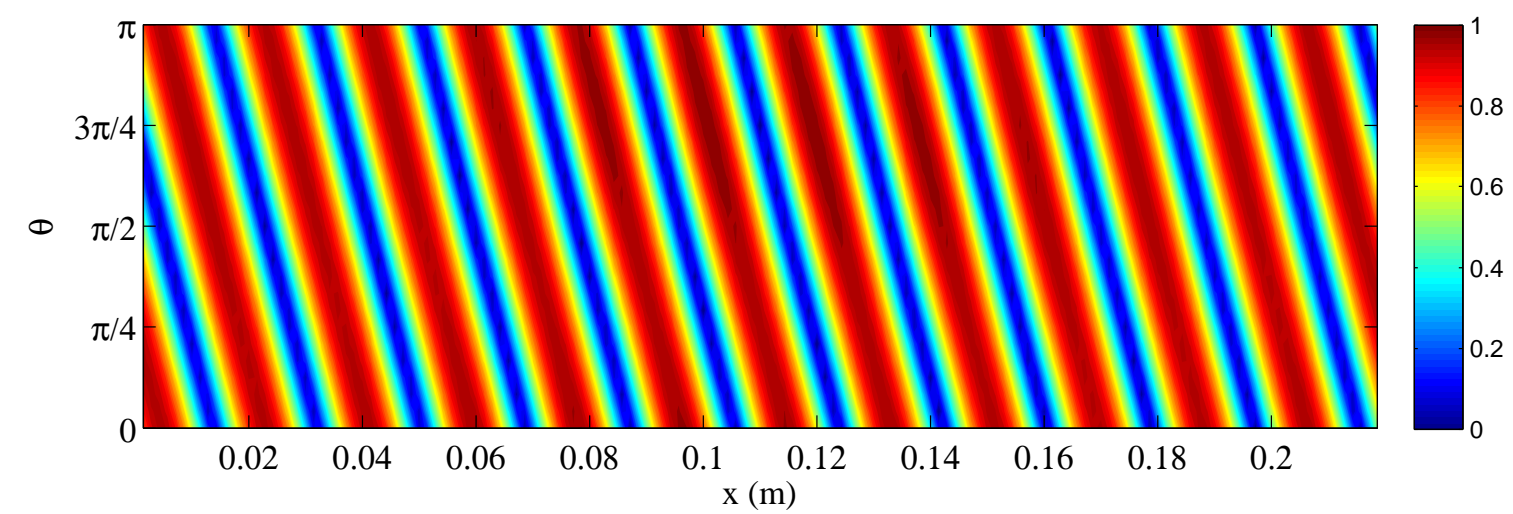

Figure 6.10: Visualization of the vibration amplitude along the top straight beam of the vibrator for $\theta \in[0, \pi]$.

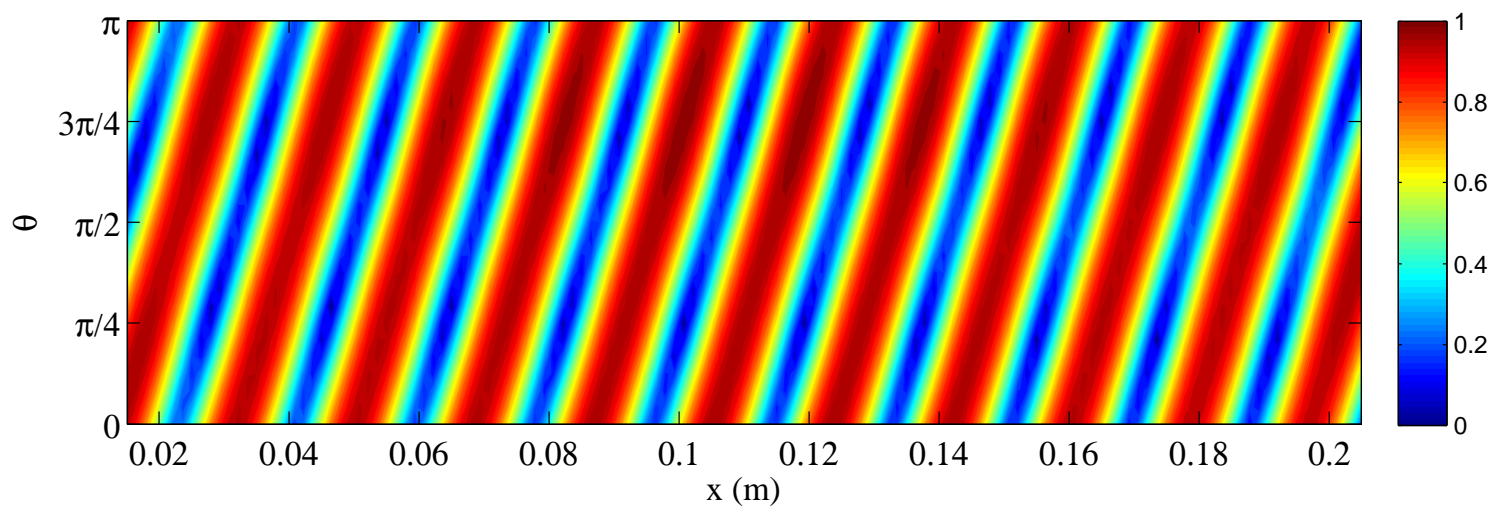

Figure 6.11: Visualization of the vibration amplitude along the bottom straight beam of the vibrator for $\theta \in[0, \pi]$. 
Those plots show that there is almost no variation of amplitude for each $\theta$ value, and that the standing wave translate linearly in function of $\theta$, as predicted section 3.1. Therefore, the displacement of the standing wave in the straight parts of the beam can be predicted by using $\Delta x=\frac{\lambda_{v} \theta}{2 \pi}$. Since here the damping is set arbitrary to $\xi=0.5 \%$, the experimental results may not be exactly as this simulation, however nonetheless this equation can give a good idea of what translation the standing wave make.

Comparing these simulations with the simulations of the acoustic levitation transportation system of section 6.1, the ring type structure presents none of the disadvantages of the prototype of (KOYAMA; NAKAMURA, 2010b), which has a non linear traveling wave and a variation in amplitude in relation to the driving phase $(\Delta \varphi)$, and no traveling wave at the ends of the plate.

\subsubsection{Analysis of the resulting acoustic field}

As for the analysis made earlier in chapter 4, a reflector is place parallel to the straight part of the vibrator, this time at a distance of $9.2 \mathrm{~mm}$, or $0.56 \lambda_{a}$, close to the value found in section 5.3. The acoustic domain discretization is 600 elements in the $x$ direction and 100 elements in the $y$ direction. Figure 6.12 presents the pressure amplitude, particle velocity amplitude and the dimensionless Gor'kov levitation potential for middle of the top part of the ring.
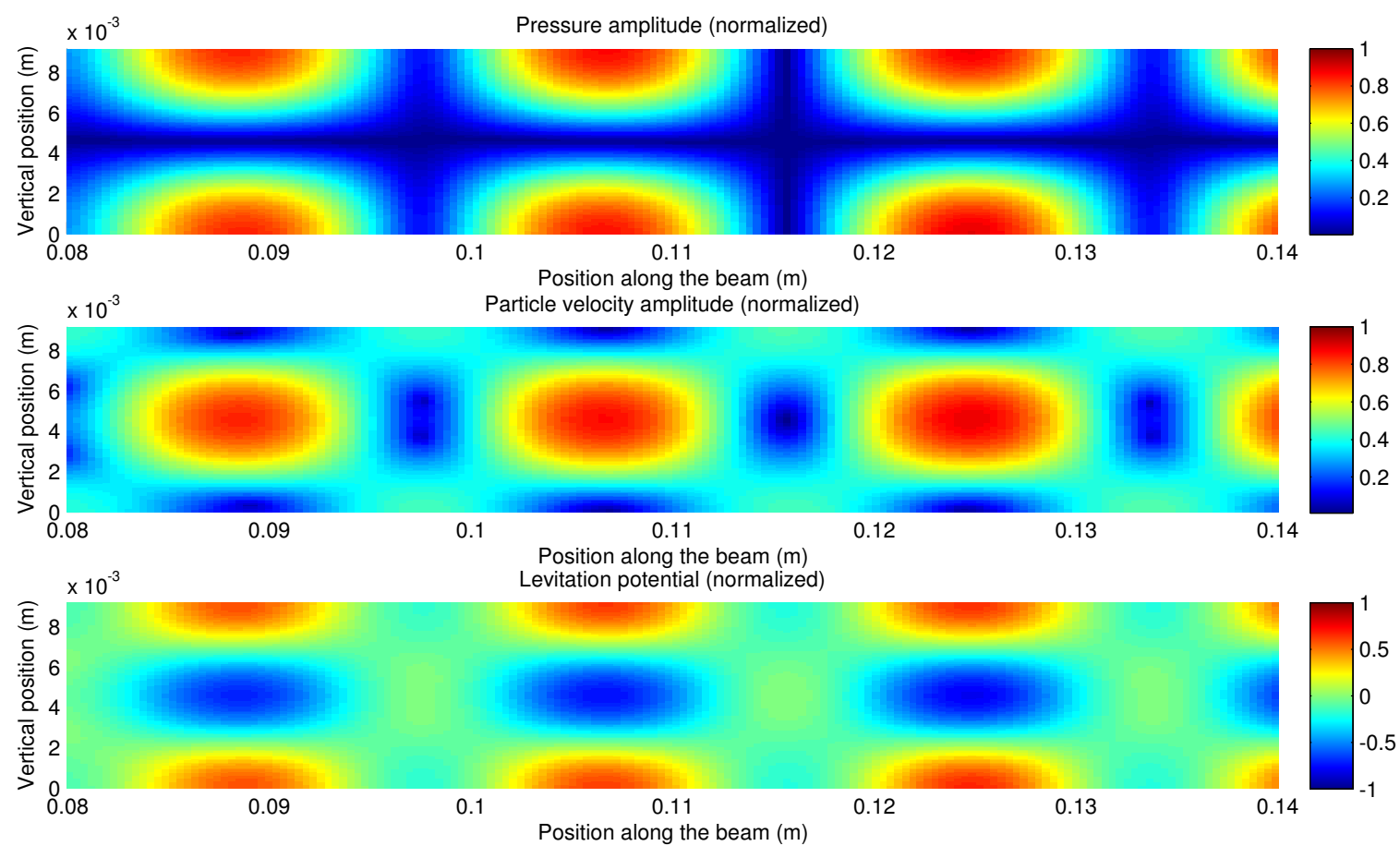

Figure 6.12: Relative vibration amplitude along the top straight part of the vibrator.

Figure 6.13 shows the displacement of the levitation potential for different values of $\theta$. As expected by the preceding plots of the vibration, the acoustic levitation potential 
translates linearly in function of $\theta$.
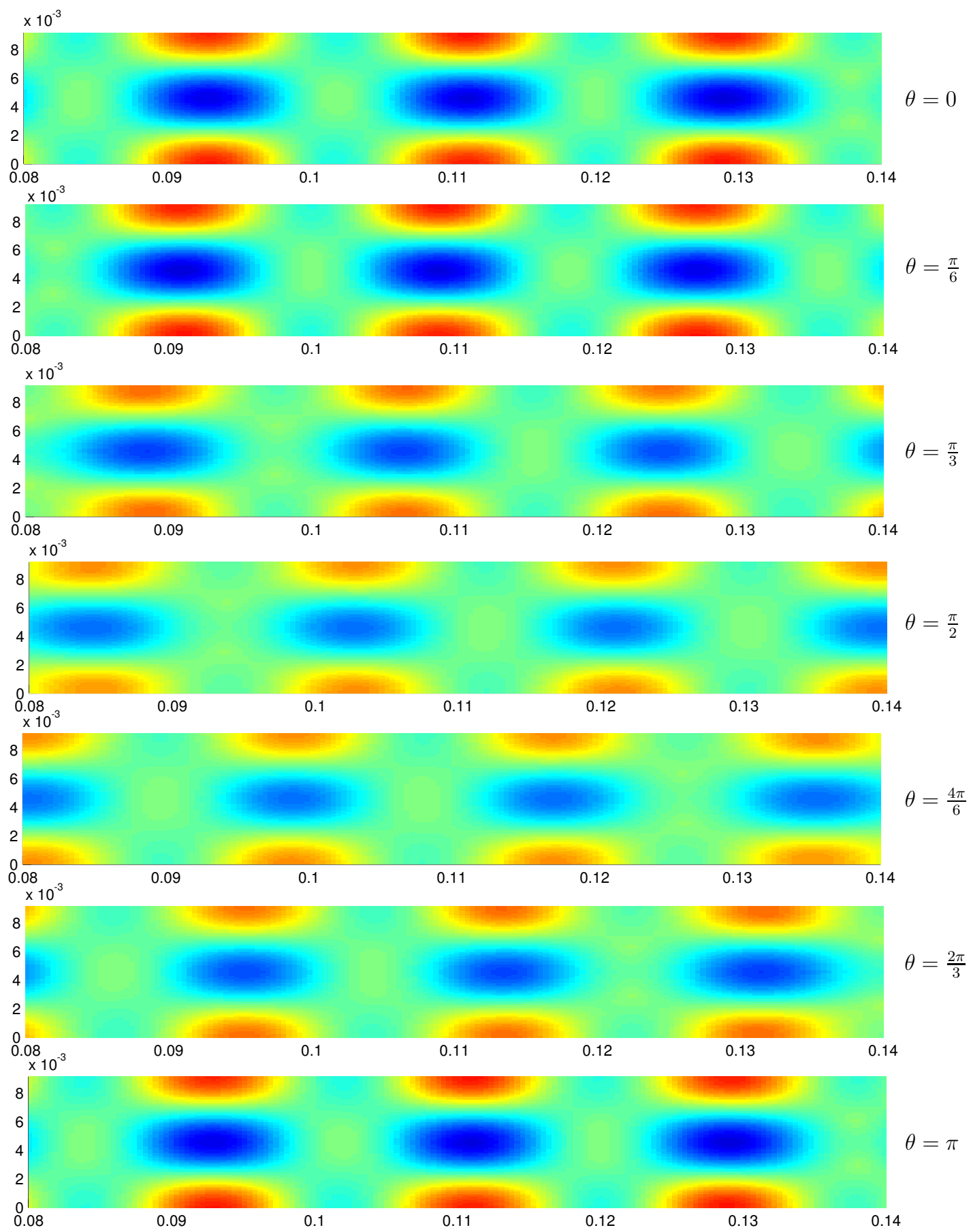

Figure 6.13: Relative dimensionless Gor'kov acoustic levitation potential for $\Delta \theta \in[0,2 \pi]$.

Therefore, it is concluded that harmonic analysis of the vibrator and its resulting acoustic field show that, in the case of the ring-type vibrator, acoustic levitation transportation of small objects is possible. The resulting translation is linked by a linear relation between the angle $\theta$ and the total displacement $\Delta x$, and there is almost no variation in amplitude during the transportation. Thus, the prototype is validated for experimental analysis. 


\section{$7 \quad$ Manufacturing of the prototype}

\subsection{Introduction}

Once all the design variables are set, a viable prototype can be made. There are various ways to manufacture it, however only the considered techniques are listed here:

- Bending and welding a plate;

- Full machining using a CNC mill;

- Full machining using a CNC wire-cut EDM machine;

\subsection{Bending and welding a plate}

This solution uses a plate of the length equals to the perimeter of the ring-type prototype. A curving roll is applied to bend the plate with the desired radius and then a MIG welding is applied to weld the plate at the intersection of the two ends to form the closed ring. The prototype is then coupled to the acoustical horns by using screws, and in order to allow impedance matching between both parts the stress distribution must be as uniform as possible. The stress distribution can be estimated by using Rotsher's pressure-cone method shown fig.7.1 (BUDYNAS-NISBETT, 2008).
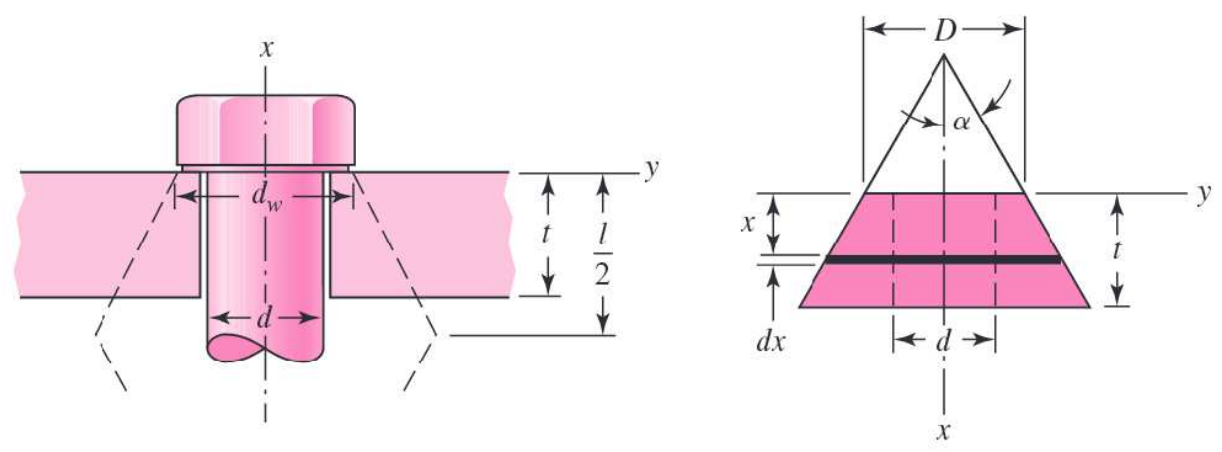

Figure 7.1: Estimated uniform pressure cone (BUDYNAS-NISBETT, 2008).

As shown in the previous chapter, the radius of the prototype must be very precise or else the superposition of the orthogonal modes cannot happen. However, the curving 
roll provides an high imprecision on both the curvature and the radius. Also, there are concerns about wave reflections at the weld joint, which could disrupt or even possibly make impossible the displacement of the standing wave inside the ring-type structure.

\subsection{Full machining using a CNC mill}

A computer numerical control (CNC) milling of an aluminium block will solve all the problems listed in the previous section, as it will allow a perfect curvature, high precision radius, and no risk of internal wave reflection since there is no joint. Furthermore, it will allow to machine the acoustical horns jointly with the ring-type structure, as one piece, and thus allowing perfect impedance matching between them. The only downside of this solution is the radius at the intersection between the horn and the ring-type structure, implied by the circular machining tool (fig.7.2). The minimum radius here, at the laboratory, would be of $3 \mathrm{~mm}$.

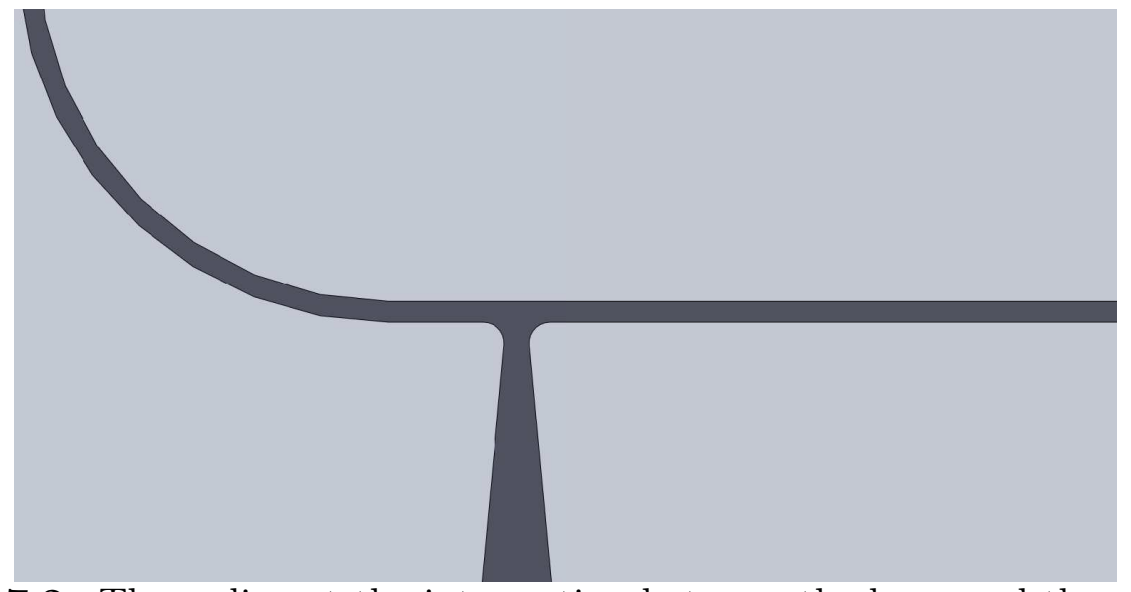

Figure 7.2: The radius at the intersection between the horn and the ring-type structure due to the milling process.

Since, as specified in the modeling chapter, the excitation of the structure must be linear and very thin, this radius could disrupt the displacement of the standing wave inside the structure.

\subsection{Full machining using a CNC wire-cut EDM machine}

A CNC wire-cut electrical discharge machining (EDM) of an aluminium block gives the same advantages as the CNC milling, such as perfect curvature, precise radius and no welding, and also allow the radius of the intersection between the horn and the structure to be much smaller $(0.5 \mathrm{~mm})$, thus allowing a linear and very thin excitation line of the 
ring-type structure.

\subsection{Manufacturing}

Since the CNC wire-cut EDM was the best way to machine the structure, it was the first choice here, however unfortunately, due to technical problem linked to the high thickness of the aluminium block (equals to the width of the structure, ' $w$ '), this operation revealed to be very difficult with the EDM machine at disposition, and thus the final prototype is machined by using a CNC mill. The CAD of the final prototype is shown fig.7.3 and a photo of the prototype during the milling is shown fig.7.4.

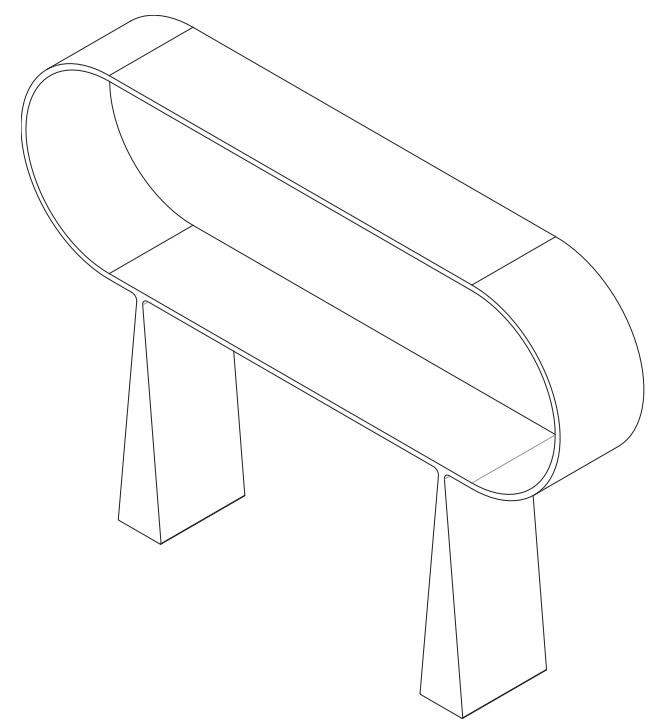

Figure 7.3: Isometric view of the CAD of the final prototype.

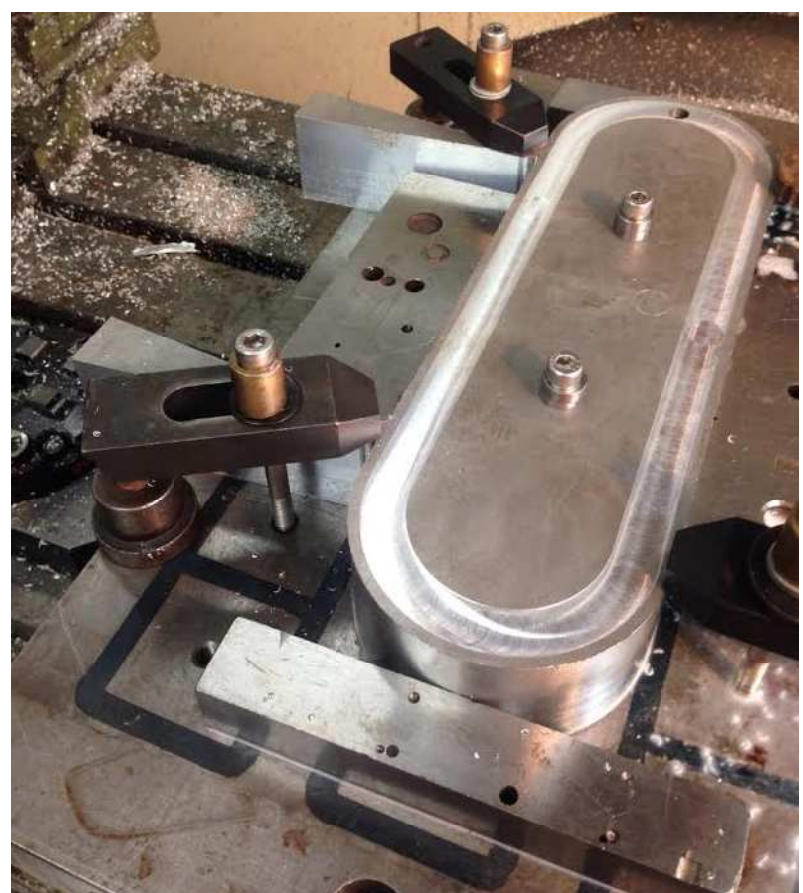

Figure 7.4: Photo of the prototype during the milling. 
The Langevin transducers are machined by using a CNC turning. A photo of the finished prototype is shown fig.7.5.

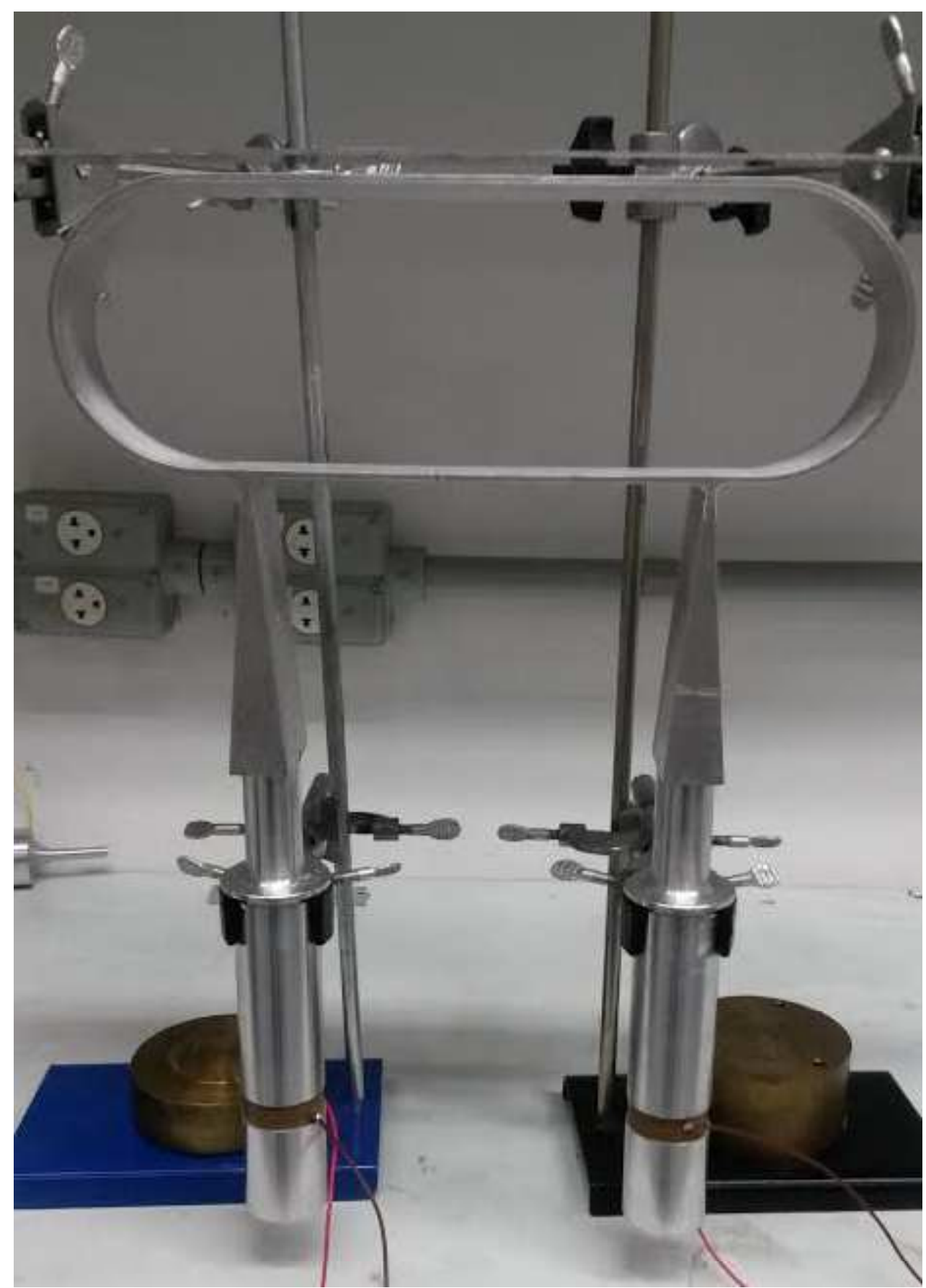

Figure 7.5: Photo of the complete prototype. 


\section{Experimental evaluation}

\subsection{Introduction}

In this chapter, the prototype modeled, designed and manufactured in the preceding chapters is evaluated experimentally. The objectives here are to determine if acoustic levitation transportation of small object is possible, if it is viable and ultimately if it comports as expected. Two different setups are needed here, one for the general case of the acoustic levitation transportation, and another one for the sole purpose of measuring the vibration distribution of the vibrator.

\subsection{Setup}

\subsubsection{Acoustic levitation transportation of particle}

In the case where the prototype is to be used as an acoustic levitation transportation system, the experimental setup is as shown in fig. 8.1.

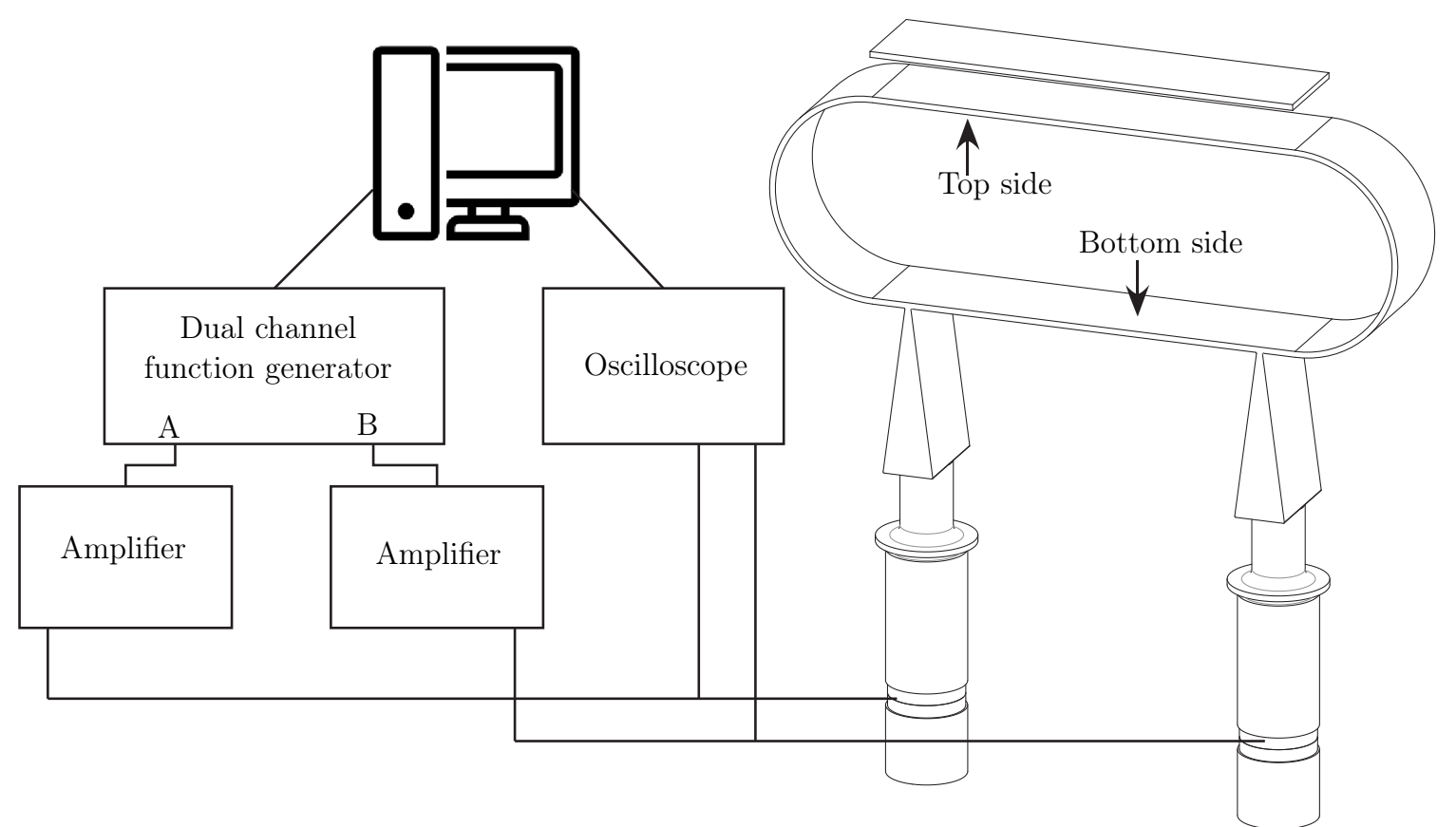

Figure 8.1: Experimental setup of the prototype for acoustic levitation transportation. 
The prototype itself is secured using a clamp on each Langevin transducer, placed at the vibration node of the amplifier, as shown previously in fig. 7.5. A metal reflector, consisting of a aluminium plate of the same width of the vibrator is secured by also using clamps or, for a more accurate positioning, using a 3 axis positioning system.

The outputs of the dual channel function generator (Agilent 33500B) are connected to high frequency amplifiers (Agilent 800A3 $10 \mathrm{kHz}-3 \mathrm{MHz}, 800 \mathrm{~W}$ ), and the outputs of those amplifiers are connected to each Langevin transducer of the prototype. The dual channel function generator enable to choose the phase difference between the two signals, which is needed here since both signals must be in phase (see section 3.1), however, the amplifiers own gain will affect this phase difference and thus an oscilloscope (Agilent DSO6052A) is used to visualize and correct accordingly the phase difference at the input of the Langevin transducers. The function generator and the oscilloscope are then both linked to a computer via ethernet, where a Matlab script controls the function generator phase and amplitude and saves the results of the oscilloscope for future plots.

The Matlab script uses the VISA Support from Instrument Control Toolbox library, which allows control and measurements of instruments of the manufacturer Agilent. The acoustic levitation transportation of small object is realized by changing the output amplitude of each phase between 0 and a maximum, where the amplitude of the output $\mathrm{A}$ is set as $A_{A}=A_{\max } \cos (\theta)$ and the amplitude of output $\mathrm{B}$ is set as $A_{B}=A_{\max } \sin (\theta)$, where $\theta$ is also the spatial phase of the standing wave inside the vibrator. Since the function generator does not work with negative amplitude, in the case where the amplitude is negative a $\pi$ temporal phase shift is made instead. Unfortunately, the function generator will activate a relay when varying to much the amplitude, and thus, during a small amount of time (almost $100 \mathrm{~ms}$ ) the output amplitude will be null and the particle will most likely fall during this time. The auto-range option of the function generator is therefore disable and the range chosen is $7 \mathrm{mV}-102 \mathrm{mV}$, meaning that a 'jump' of $14 \mathrm{mV}$ will occur when changing the phase sign (6.9\% of the whole range). The Matlab script used here is available in appendix C.1.

Also, even if the Langevin transducer are made to be identical, in the end there is a difference of working frequency of more or less $100 \mathrm{~Hz}$ between them, meaning that the vibration amplitude of one transducer is different of the other for the same tension input. One solution is to adjust the maximum amplitude of the signal output of the function generator in order to obtain the same scale of vibration amplitude for both Langevin transducers. 


\subsubsection{Vibration measurement}

In the case where the vibration amplitude of the vibrator needs to be measured, the experimental setup is as shown in fig. 8.2.

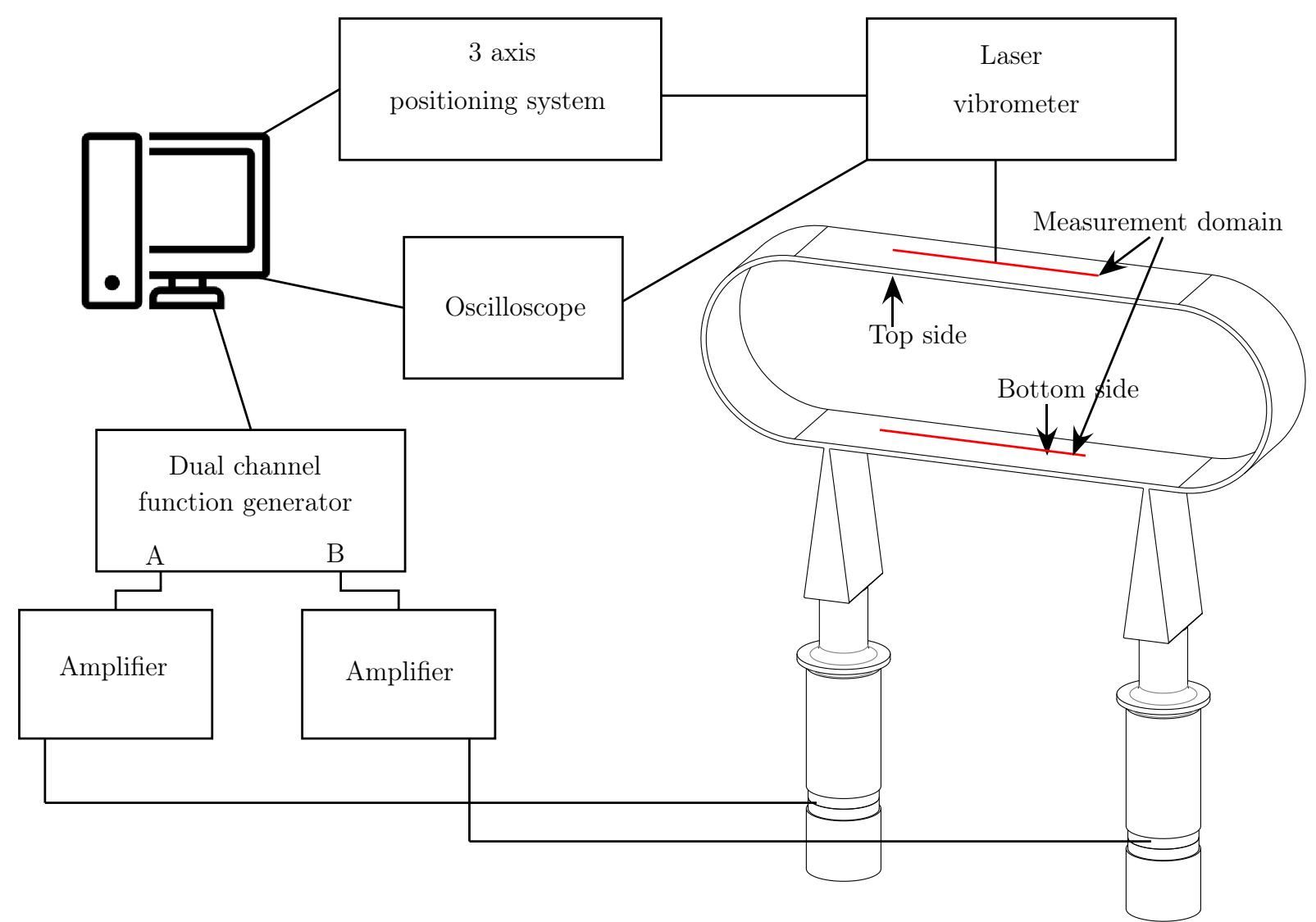

Figure 8.2: Experimental setup for the vibration amplitude measurement.

The setup of the prototype itself is identical as before, with a dual channel function generator and two amplifiers, however, this time a 3 axis positioning system (Thorlabs APT Stepper Motor Control Module with 3 NRT150 Motorized Linear Translation Stage) and a laser vibrometer (Polytec OFV-5000) are used to measure the vibration amplitude of the vibrator. The phase difference between the two transducers is adjusted before the experiment, and then the oscilloscope is used to read the results of the laser vibrometer. The vibration amplitude measurement is made at the middle of the width of the vibrator, for a maximum length of $120 \mathrm{~mm}$ around the middle of the total length of the straight part of the vibrator, and for both the straight parts. In further reference, the bottom side of the vibrator is the straight part of the vibrator with the acoustical horns and the top side is the other straight part.

The prototype needs to be vertical during the measurement, as if it is set horizontal the torque generated by its own mass changes the bandwidth of the transducers and makes difficult to control the prototype. Photos of the setup for both cases (measurements of the top side and the bottom side) are shown in fig. 8.3. 


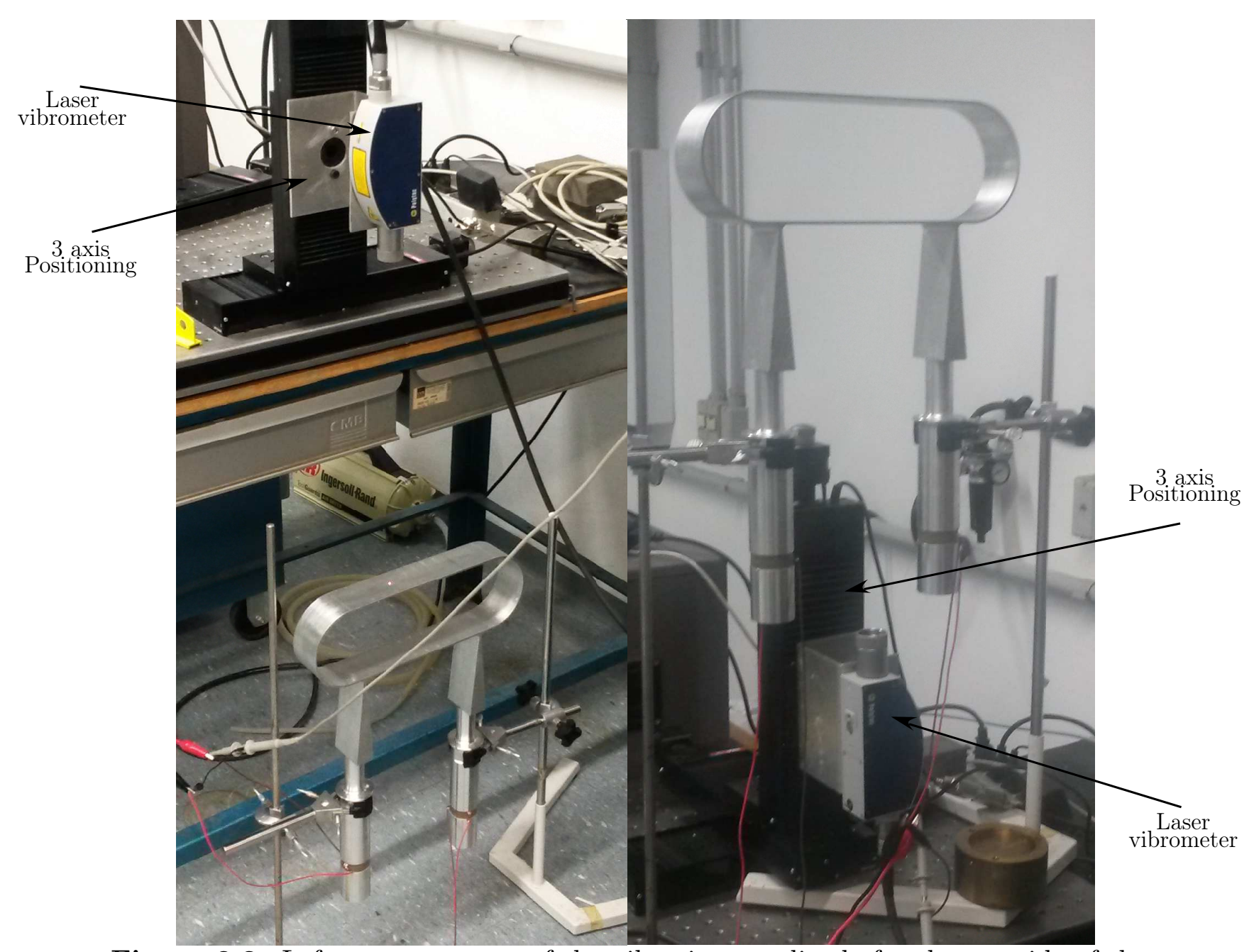

Figure 8.3: Left: measurement of the vibration amplitude for the top side of the vibrator; Right: measurement of the vibration amplitude for the bottom side of the vibrator.

As before, the full experiment is controlled by using a Matlab script. The positioning system is controlled by using the Microsoft ActiveX library to communicate with the Thorlabs control software. The objective here is to measure the vibration repartition along the vibrator for various values of $\theta$ to visualize the traveling wave. The full script used here is available in appendix C.2

\subsection{Results}

\subsubsection{Acoustic levitation transportation of particle}

The experimental working frequency of the prototype is $21,600 \mathrm{~Hz}( \pm 20 \mathrm{~Hz}$, depending mostly of the ambient temperature), with an average wavelength in its straight part of $\lambda_{v}=36.2 \mathrm{~mm}$, which are both very close to the calculated values $(21,348 \mathrm{~Hz}$ and $36.7 \mathrm{~mm})$. 
For most part of the experiments, the reflector is placed using the 3 -axis positioning system describe in section 8.2.2, as if not placed perfectly parallel to the vibrator the particle cannot be transported over long distance. Also, the distance between the vibrator and the reflector must be adjusted to the tenth of $\mathrm{mm}$ to successfully levitates small objects, and can vary from one experiment to another, depending mostly of the ambient conditions. This problems are mostly the results of placing the reflector close to only half of the acoustical wavelength (i.e. $\frac{\lambda_{a}}{2}$ ), as when using a full wavelength the error margin allowed is more comfortable, but the mass of the levitated particle has to be very small. Experimentally, the distance between the reflector and the vibrator is $9.2 \pm 0.2 \mathrm{~mm}$.

Acoustic levitation transportation of a small spherical polystyrene particles $(\varnothing<4$ $\mathrm{mm}$ ) is obtained successfully along the straight parts of the ring-type vibrator, with the exception of on top of the junction between the mechanical horns and the vibrator. Figure 8.4 shows the trajectory followed by the particle at the middle of the straight part of the bottom of the vibrator, between the two horns, for $\theta \in[0 ; 2 \pi]$ with $\dot{\theta}=0.35 \mathrm{rad} / \mathrm{s}$, where the superimposed photographs are taken at each second.

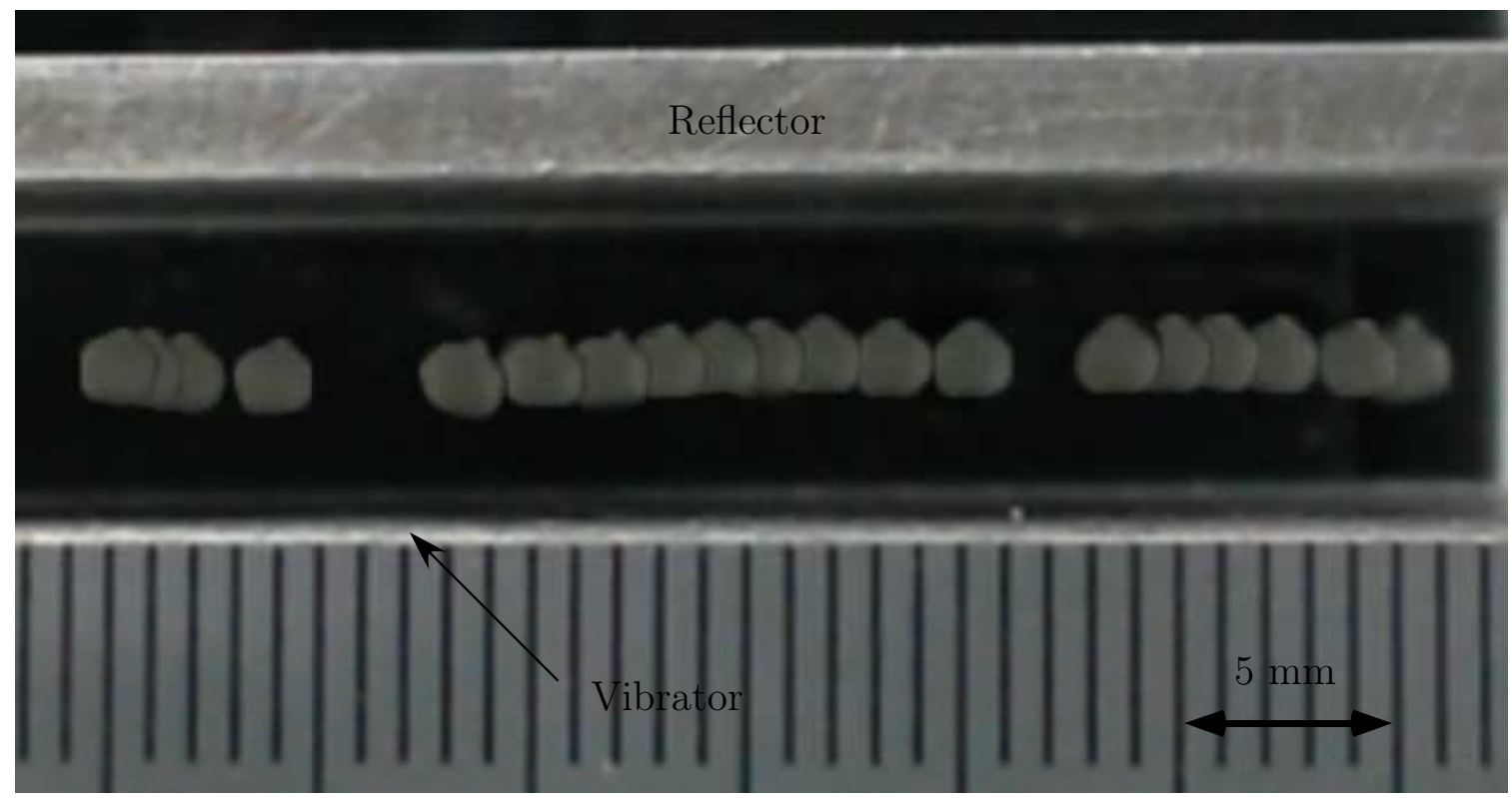

Figure 8.4: Trajectory of the levitated particle.

Figure 8.4 shows that the particle translates with an almost linear trajectory. The "jumps" that the particle suffers are caused by the scaling limitation of the function generator (the amplitude of the signals sent to the amplifiers is between $7 \mathrm{mV}$ and 102 $\mathrm{mV}$ instead of $0 \mathrm{mV}$ and $102 \mathrm{mV}$, as explained in section 8.2.1). Because of this, acoustic levitation transportation of liquid droplet is not possible, with the droplet falling or exploding at those "jumps", however the liquid droplets are successfully levitated and transported in the domain $\theta \in\left[n \frac{\pi}{2},(n+1) \frac{\pi}{2}\right] ; n \in \mathbb{N}$. 
The plot from fig. 8.5 shows the relation between the distance traveled by a particle $\Delta x$ and the variable $\theta$ in the case of an almost static experiment $(\dot{\theta} \approx 0$ and the auto-range option of the function generator activated). The experiment shows that the distance traveled by the particle in relation to $\theta$ is very close to the linear model $\Delta x=\frac{\theta}{k}$.

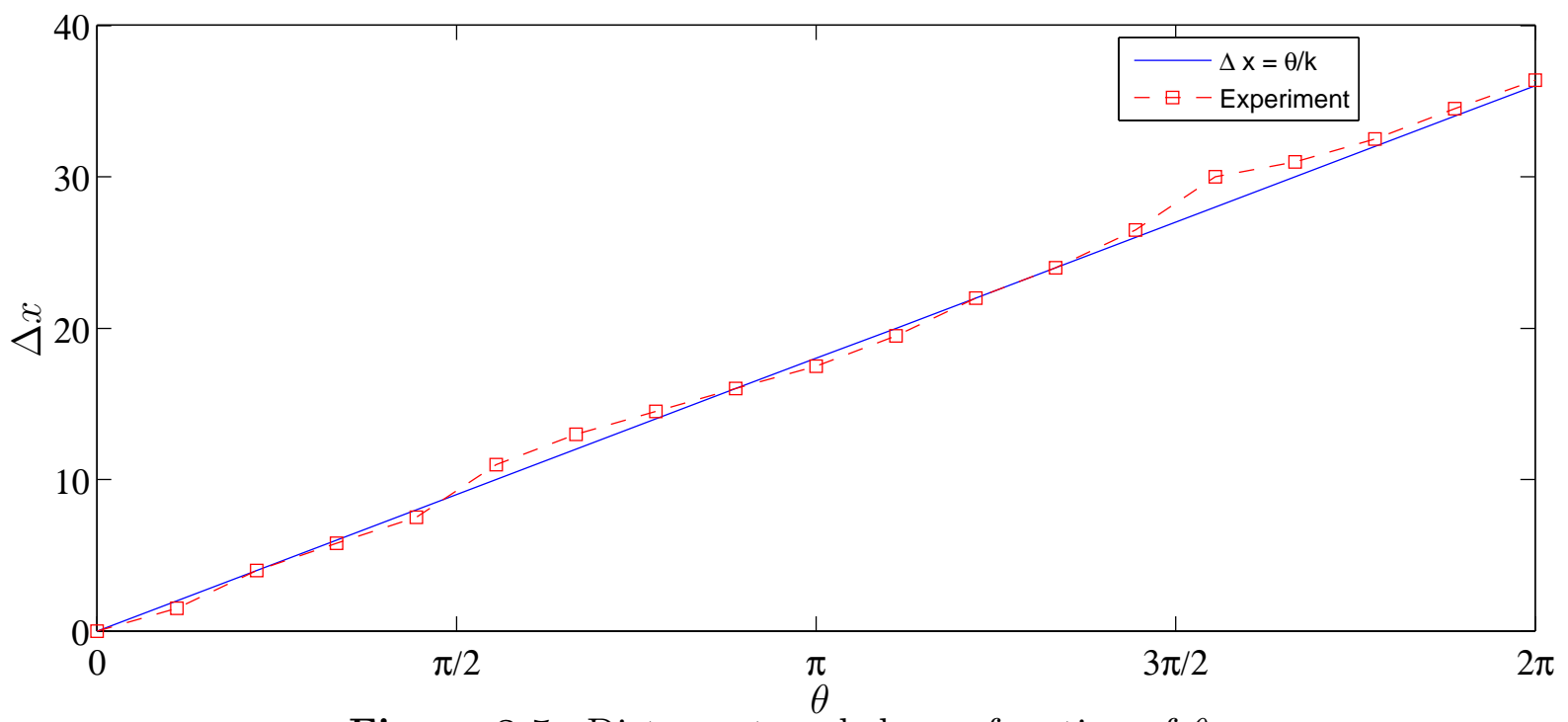

Figure 8.5: Distance traveled as a function of $\theta$.

\subsubsection{Vibration measurement}

Figures 8.6 and 8.7 show the vibration amplitude $\xi$ in $\mathrm{m}$, measured by a laser vibrometer, at the middle of the width of the vibrator for various values of $\theta$ between 0 and $\pi$. This allows us to see the spatial phase shift of the standing wave in the vibrator in relation to $\theta$.

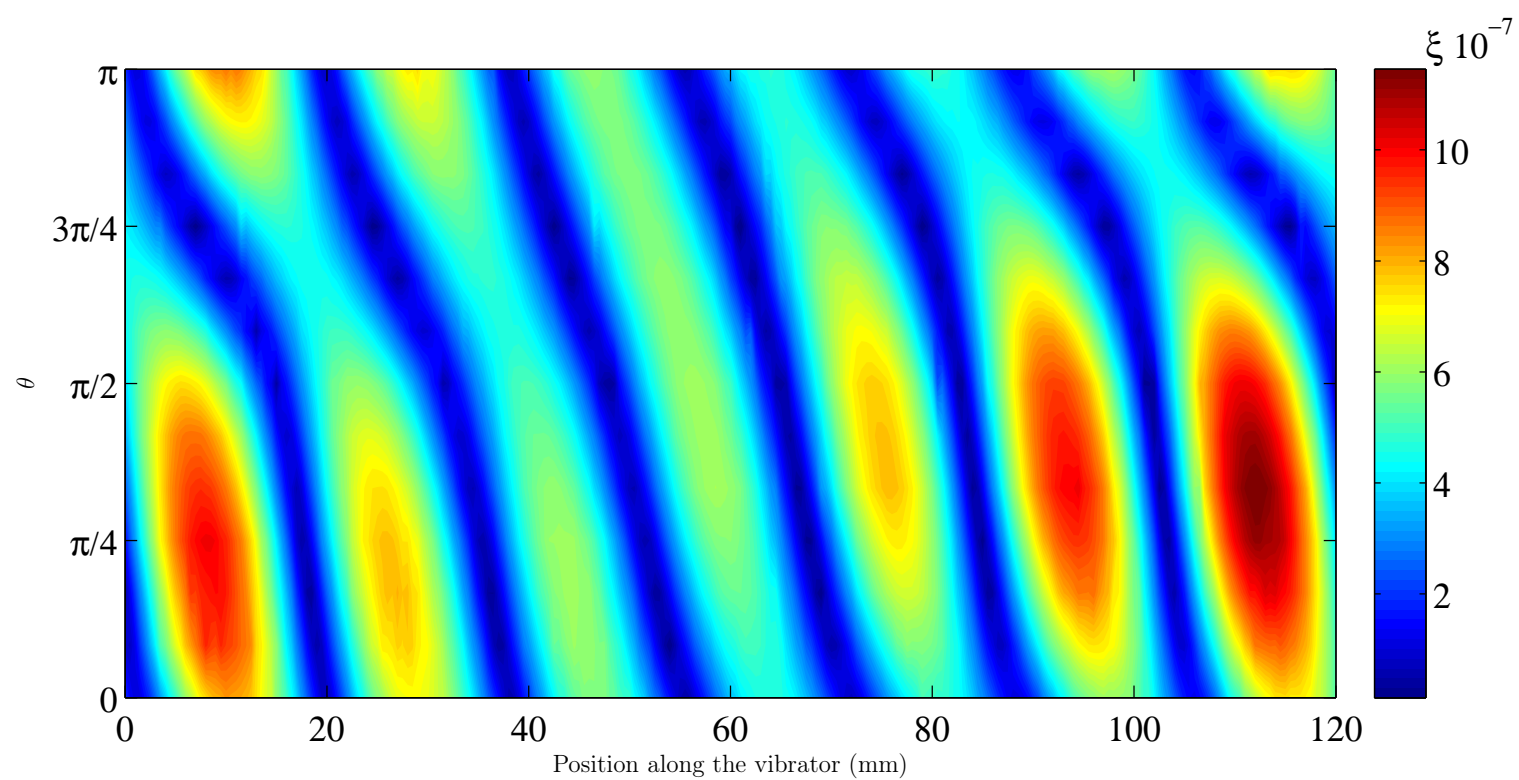

Figure 8.6: Amplitude of the standing wave at the bottom of the vibrator for $\theta \in[0, \pi]$ measured by the laser vibrometer. 


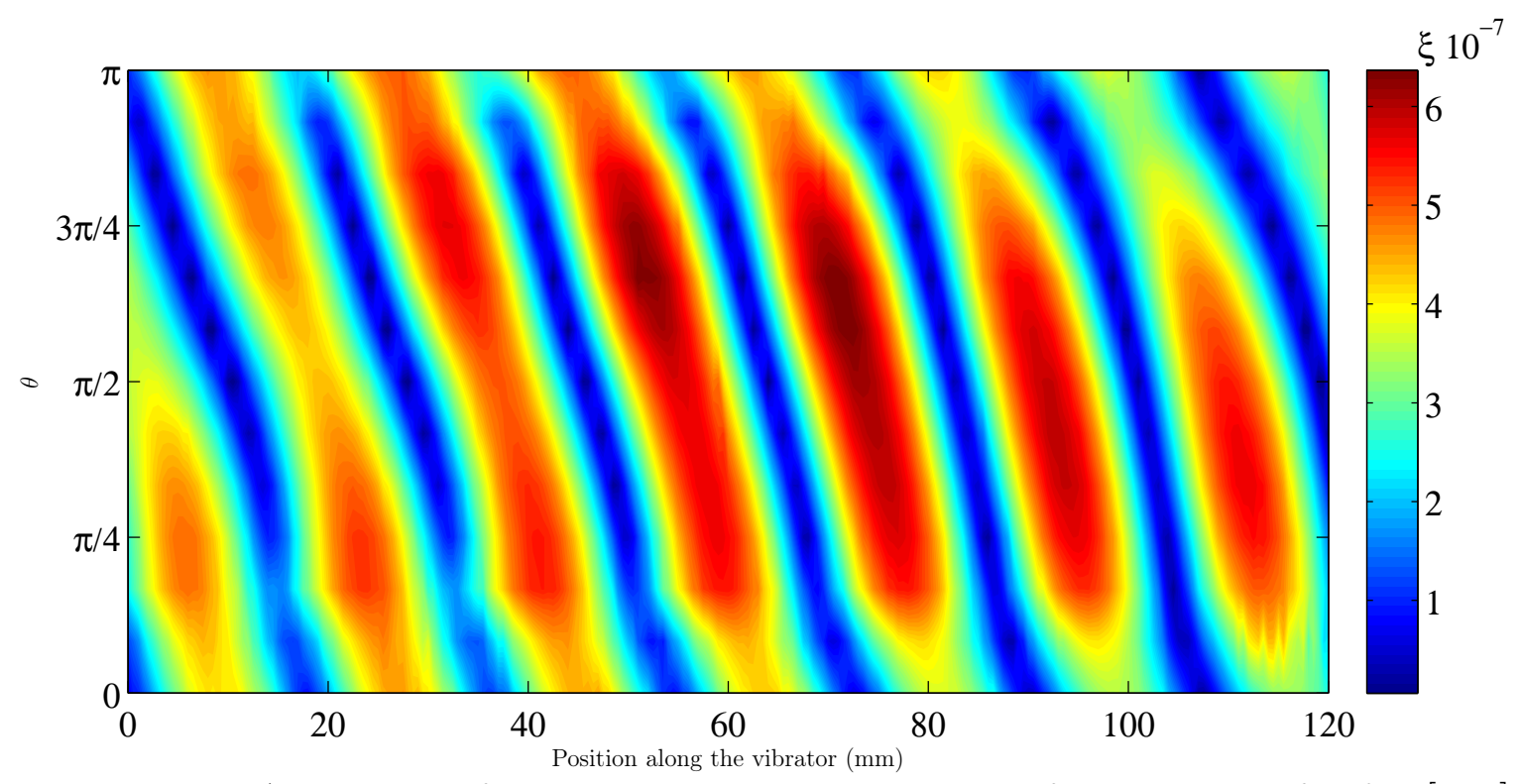

Figure 8.7: Amplitude of the standing wave at the top of the vibrator for $\theta \in[0, \pi]$ measured by the laser vibrometer.

The relation between the spatial phase shift and the value of $\theta$, is, as expected, almost linear with a proportional coefficient equal to $\Delta x=\frac{\theta}{k}$. Unfortunately, this is not always true, as closer to the excitation points, the less linear is the relation between the spatial phase shift and $\theta$. This can be explained by the wave reflections at the junctions between the horns and the vibrator, as in the modeling process those are considered as a line of infinitesimal width.

Also, the parts close to the acoustical horns have a high vibration amplitude than everywhere else. This is mainly due to the natural damping of the structure and is very hard to model without an empirical value of the damping. 


\section{Conclusion and future work}

In this dissertation, a full modeling of an already existing acoustic levitation transportation system using a beam vibrator is made successfully, and thus this model could be use for the design of a specific acoustic levitation transportation system of the same type. The modeling and analysis of this system was also required in order to get a better understanding of acoustic levitation transportation. However, this system presents three major disadvantage: a non linear traveling wave, a fluctuating amplitude in relation to the spatial phase of the wave and the impossibility to displace the particle at the ends of the vibrator, behind its transducers. Therefore, a new way of accomplishing acoustic levitation long distance linear transportation of small object by using a ring-type vibrator was studied.

For certain values of the ratio length/radius $\left(\frac{L}{R}\right)$ of the ring-type vibrator, two pseudo orthogonal modes can exist at the same frequency, allowing travelling wave along the vibrator and thus acoustic levitation transportation. This ratio need to be carefully designed, as if not precisely set the travelling wave cannot be generated. Therefore, a full analytical and numerical modeling of a ring-type vibrator were successfully made in order to design a prototype of an acoustic levitation linear transportation system using a ring-type vibrator. The designed vibrator was then analysed numerically and experimentally, showing good agreement between them.

The ring-type structure presents none of the disadvantage of the beam vibrator, as proven in this dissertation, as it has a linear traveling wave with almost constant amplitude, and small object can be displaced for the total length of its straight part, with the exception of the side where the transducers are present. Particle of polystyrene and water were successfully levitated and transported along the straight parts of the vibrator. The maximum transportation distance achieved for solid objects by the prototype is 220 $\mathrm{mm}$.

In future work, the transport of particle from one acoustic levitation transportation system to another can be studied, in order to achieve greater transportation distance. This may be done, but not exclusively, by using the curved part of the ring-type vibrator and 
designing a particular reflector that can join the curved part of two ring-type vibrators. Another idea is to use the method described in (FORESTI; NABAVI, 2013), where the particle is transferred from one acoustic levitation system to another by decreasing the pressure amplitude of the sender and increasing the pressure amplitude of the receiver. 


\section{References}

ABDULLAH, A.; SHAHINI, M.; PAK, A. An approach to design a high power piezoelectric ultrasonic transducer. Journal of Electroceramics, v. 22, n. 4, p. 369-382, jan. 2009. ISSN 1385-3449. Disponível em: <http://link.springer.com/10.1007/s10832-007-9408-8 http://link.springer.com/article/10.1007/s10832-007-9408-8>.

AMIN, S. G.; AHMED, M. H. M.; YOUSSEF, H. A. On the forces acting on a small particle in an acoustical field in an ideal fluid. Journal of Materials Processing Technology, v. 55, p. 254-260, 1995.

ANDRADE, M. Finite element analysis and optimization of a single-axis acoustic levitator. IEEE Transactions on Ultrasonics, Ferroelectrics, and Frequency Control, v. 57, n. 2, p. 469-479, 2010. Disponível em: $<\mathrm{http}$ ://ieeexplore.ieee.org/xpls/abs_all.jsp?arnumber $=5417206>$.

ANDRADE, M. A. B.; ADAMOWSKI, J. C. Matrix Method for Acoustic Levitation Simulation. IEEE Transactions on Ultrasonics, Ferroelectrics, and Frequency Control, v. 58, n. 8, p. 1674-1683, 2011.

APFEL, R.; TIAN, Y.; JANKOVSKY, J.; SHI, T.; CHEN, X.; HOLT, R.; TRINH, E.; CROONQUIST, A.; THORNTON, K.; Sacco, Jr., A.; COLEMAN, C.; LESLIE, F.; MATTHIESEN, D. Free Oscillations and Surfactant Studies of Superdeformed Drops in Microgravity. Physical Review Letters, v. 78, n. 10, p. 1912-1915, 1997.

BARMATZ, M. Acoustic radiation potential on a sphere in plane, cylindrical , and spherical standing wave fields a ). J. Acoust. Soc. Am., v. 77, n. March, p. 928-945, 1985.

BERENGER, J.-p. Perfectly Matched Layer for the FDTD Solution of Wave-Structure Interaction Problems. v. 44, n. 1, p. 110-117, 1996.

BUCKS, K.; MULLER, H. Uber einige Beobachtungen an schwingenden Piezoquarzen und ihrem Schallfeld. 1933.

BUDYNAS-NISBETT. Shigley's Mechanical Engineering Design, Eigth Edition. [S.1.: s.n.], 2008.

CHIDAMPARAM, P.; LEISSA, A. Vibrations of planar curved beams, rings, and arches. Appl Mech Rev, v. 46, n. 9, p. 467-483, 1993.

CLAIR, H. W. S. Agglomeration of Smoke, Fog, or Dust Particles by Sonic Waves. Industrial and Engineering Chemestry, v. 41, n. 11, 1949.

COOK, R. D.; MALKUS, D. S.; PLESHA, M. E.; WITT, R. J. Concepts and applications of finite element analysis. 4th editio. ed. [s.n.], 2002. 388-390 p. Disponível em:

$<$ http://scholar.google.com/scholar?hl=en\&btnG $=$ Search\&q=intitle:Concepts + and + Applications http://scholar.google.com/scholar?hl=en\&btnG=Search\&q=intitle:Concepts + and + applications + of 
FORESTI, D.; BJELOBRK, N. Investigation of a line-focused acoustic levitation for contactless transport of particles. Journal of Applied ..., v. 109, n. 9, p. 093503, 2011. ISSN 00218979. Disponível em: <http://scitation.aip.org/content/aip/journal/jap/109/9/10.1063/1.3571996>.

FORESTI, D.; NABAVI, M. Acoustophoretic contactless transport and handling of matter in air. Proceedings of the ..., v. 110, n. 31, p. 12549-54, jul. 2013. ISSN 1091-6490. Disponível em: $<$ http://www.pubmedcentral.nih.gov/articlerender.fcgi?artid=3732964\&tool=pmcentrez\&rendertyp http://www.pnas.org/content/110/31/12549.short>.

GOR'KOV, L. P. On the forces acting on a small particle in an acoustical field in an ideal fluid. Soviet Physics - Doklady, v. 6, p. 773-775, 1962.

GORMAN, D. J. Free Vibration Analysis of Beams and Shaft. [S.l.: s.n.], 1975. 7-8 p.

HANSON, A. R.; DOMICH, E. G.; ADAMS, H. S. Acoustical Liquid Drop Holder. The Review of Scientific Instruments, v. 35, n. 8, 1964.

IHLENBURG, F. Finite Element Analysis of Acoustic Scattering. Springer-Verlag, New York, v. 132, 1998. Disponível em: <http://link.springer.com/10.1007/b98828>.

JOHNSON, S. Notes on perfectly matched layers (PMLs). Lecture notes, Massachusetts Institute of ..., n. August 2007, p. 1-18, 2008. Disponível em: <http://www-math.mit.edu/ stevenj/18.369/spring09/pml.pdf >.

KASHIMA, R.; MURAKAMI, S.; KOYAMA, D. Design of a Junction for a Noncontact Ultrasonic Transportation System. IEEE Transactions on Ultrasonics, Ferroelectrics, and Frequency Control, v. 61, n. 6, 2014.

KING, L. V. On the Acoustic Radiation Pressure on Spheres. Proceedings of the Royal Society A: Mathematical, Physical and Engineering Sciences, v. 147, n. 861, p. 212-240, nov. 1934. ISSN 1364-5021. Disponível em: <http://rspa.royalsocietypublishing.org/cgi/doi/10.1098/rspa.1934.0215>.

KINSLER, L. E.; FREY, A. R.; COPPENS, A. B.; SANDERS, J. V. Fundamentals of Acoustics. John Wiley E Sons, n. 3 ed., 1982.

KOYAMA, D.; NAKAMURA, K. Noncontact ultrasonic transportation of small objects in a circular trajectory in air by flexural vibrations of a circular disc. IEEE Transactions on Ultrasonics, Ferroelectrics, and Frequency Control, v. 57, n. 6, p. 1434-1442, 2010. Disponível em: <http://ieeexplore.ieee.org/xpls/abs_all.jsp?arnumber=5480185>.

KOYAMA, D.; NAKAMURA, K. Noncontact ultrasonic transportation of small objects over long distances in air using a bending vibrator and a reflector. IEEE Transactions on Ultrasonics, Ferroelectrics, and Frequency Control, v. 57, n. 5, p. 1152-1159, 2010. Disponível em: <http://ieeexplore.ieee.org/xpls/abs_all.jsp?arnumber $=5456264>$.

KOYAMA, D.; NAKAMURA, K.; UEHA, S. A stator for a self-running, ultrasonically-levitated sliding stage. IEEE Transactions on Ultrasonics, Ferroelectrics, and Frequency Control, v. 54, n. 11, p. 2337-2343, 2007. Disponível em: $<\mathrm{http}$ ://ieeexplore.ieee.org/xpls/abs_all.jsp?arnumber $=4399708>$. 
KOZUKA, T.; TUZIUTI, T. Control of position of a particle using a standing wave field generated by crossing sound beams. IEEE Transactions on Ultrasonics, Ferroelectrics, and Frequency Control ..., v. 1, p. 657-660, 1998. Disponível em: $<$ http://ieeexplore.ieee.org/xpls/abs_all.jsp?arnumber $=762234>$.

LENSHOF, A.; MAGNUSSON, C.; LAURELL, T. Acoustofluidics 8: Applications of acoustophoresis in continuous flow microsystems. Lab Chip, The Royal Society of Chemistry, v. 12, n. 7, p. 1210-1223, 2012. Disponível em: <http://dx.doi.org/10.1039/C2LC21256K>.

LIU, X.; SHI, D.; CIVET, Y.; PERRIARD, Y. Modelling and Optimal Design of a Ring-type Structure for the Generation of a Traveling Wave. International Conference on Electrical Machines and Systems, p. 1286-1291, 2013.

LIU, Y.; HU, J. Trapping of particles by the leakage of a standing wave ultrasonic field. Journal of Applied Physics, v. 106, n. 3, p. 034903, 2009. ISSN 00218979. Disponível em: $<$ http://scitation.aip.org/content/aip/journal/jap/106/3/10.1063/1.3169517>.

OCHIAI, Y.; HOSHI, T.; REKIMOTO, J. Three-dimensional mid-air acoustic manipulation by ultrasonic phased arrays. PloS one, v. 9, n. 5, p. e97590, jan. 2014. ISSN 1932-6203. Disponível em: <http://www.pubmedcentral.nih.gov/articlerender.fcgi?artid=4029622\&tool=pmcentrez\&rendertyp

PUSKAR, L.; TUCKERMANN, R.; FROSCH, T.; POPP, J. Raman acoustic levitation spectroscopy of red blood cells and Plasmodium falciparum trophozoites. Lab Chip, v. 7, n. 9, p. 1125-1131, set. 2007. ISSN 1473-0197. Disponível em: <http://www.ncbi.nlm.nih.gov/pubmed/17713610 http://pubs.rsc.org/en/content/articlehtml/2007/lc/b706997a>.

REINHART, G.; HOEPPNER, J. Non-Contact Handling Using High-Intensity Ultrasonics. CIRP Annals - Manufacturing Technology, v. 49, n. 1, p. 5-8, jan. 2000. ISSN 00078506. Disponível em: $<$ http://www.sciencedirect.com/science/article/pii/S0007850607628844>.

SANTESSON, S.; NILSSON, S. Airborne chemistry: acoustic levitation in chemical analysis. Analytical and bioanalytical chemistry, v. 378, n. 7, p. 1704-1709, abr. 2004. ISSN 1618-2642. Disponível em: <http://www.ncbi.nlm.nih.gov/pubmed/14762640 http://link.springer.com/article/10.1007/s00216-003-2403-2>.

SEAH, S. A.; DRINKWATER, B. W.; CARTER, T.; MALKIN, R.; SUBRAMANIAN, $\mathrm{S}$. Dexterous ultrasonic levitation of millimeter-sized objects in air. IEEE transactions on ultrasonics, ferroelectrics, and frequency control, v. 61, n. 7, p. 1233-6, jul. 2014. ISSN 1525-8955. Disponível em: <http://www.ncbi.nlm.nih.gov/pubmed/24960712>.

SEEMANN, W. A Linear Ultrasonic Traveling Wave Motor of the Ring Type. Smart Mater. Struct., v. 5, p. 361-368, 1996.

WANG, T.; TRINH, E.; CROONQUIST, A.; ELLEMAN, D. Shapes of rotating free drops: Spacelab experimental results. Physical review letters, v. 56, p. 3-6, 1986. Disponível em: <http://journals.aps.org/prl/abstract/10.1103/PhysRevLett.56.452>.

XIE, W.; CAO, C.; Lü, Y.; WEI, B. Levitation of Iridium and Liquid Mercury by Ultrasound. Physical Review Letters, v. 89, n. 10, p. 104304, ago. 2002. ISSN 0031-9007. Disponível em: <http://link.aps.org/doi/10.1103/PhysRevLett.89.104304>. 
XIE, W.; CAO, C.; Lü, Y.; HONG, Z.; WEI, B. Acoustic method for levitation of small living animals. Applied physics letters, v. 89, n. 21, p. 214102, 2006. ISSN 00036951. Disponível em: <http://scitation.aip.org/content/aip/journal/apl/89/21/10.1063/1.2396893>.

XIE, W.; WEI, B. Parametric study of single-axis acoustic levitation. Applied Physics Letters, v. 79, n. 6, p. 881-883, 2001. ISSN 00036951. Disponível em: $<$ http://scitation.aip.org/content/aip/journal/apl/79/6/10.1063/1.1391398>.

XIE, W.; WEI, B. Dependence of acoustic levitation capabilities on geometric parameters. Physical Review E, v. 66, n. 2, p. 026605, ago. 2002. ISSN 1063-651X. Disponível em: <http://link.aps.org/doi/10.1103/PhysRevE.66.026605 http://journals.aps.org/pre/abstract/10.1103/PhysRevE.66.026605>.

XIE, W.; WEI, B. Temperature dependence of single-axis acoustic levitation. Journal of applied physics, v. 93, n. 5, p. 3016, 2003. ISSN 00218979. Disponível em: $<$ http://scitation.aip.org/content/aip/journal/jap/93/5/10.1063/1.1540232>.

XIE, W.; WEI, B. Sound field inside acoustically levitated spherical drop. Applied physics letters, v. 90, n. 20, p. 204104, 2007. ISSN 00036951. Disponível em: <http://scitation.aip.org/content/aip/journal/apl/90/20/10.1063/1.2741051 http://ieeexplore.ieee.org/xpls/abs_all.jsp?arnumber $=4826968>$. 


\section{Appendix A}

\section{A.1 MatLab implementation}

\section{A.1.1 Program structure}

First, two struct-type variables are created and contain all data related to the beam and to the fluid. Both struct variables are divided with four sub-struct, as shown in fig. A.1.

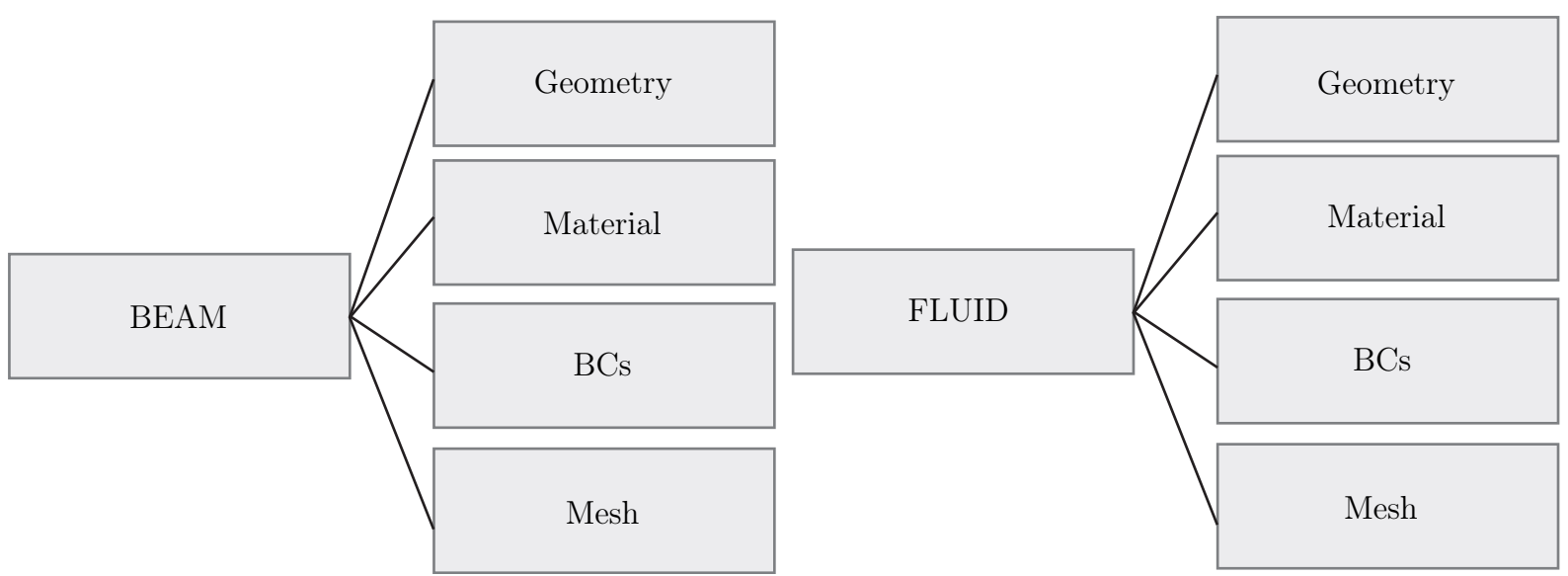

Figure A.1: Composition of the two main struct variables of the FEM.

- The geometry sub-struct inform about the geometry of each domain, such as the length, width and thickness.

-The material sub-struct contain the material property, such as the density, Young's modulus and Rayleigh's damping coefficients for the beam and the density and speed of sound for the fluid.

- The BCs (boundary conditions) sub-struct inform about the loads and boundary conditions of each domain.

-The mesh sub-struct contain all the data relative to the discretization of each domain.

A diagram showing all the steps of a complete analysis of the entire model is shown fig. A.2. The first thing to do is the initialization of each variable of the model, so the 
geometry, material and BCs sub-struct have to be fully filled. In the mesh sub-struct the initial seed of the discretization also have to be informed. Each step after the variables initialization represents a MatLab function which are detailed in the next sections.

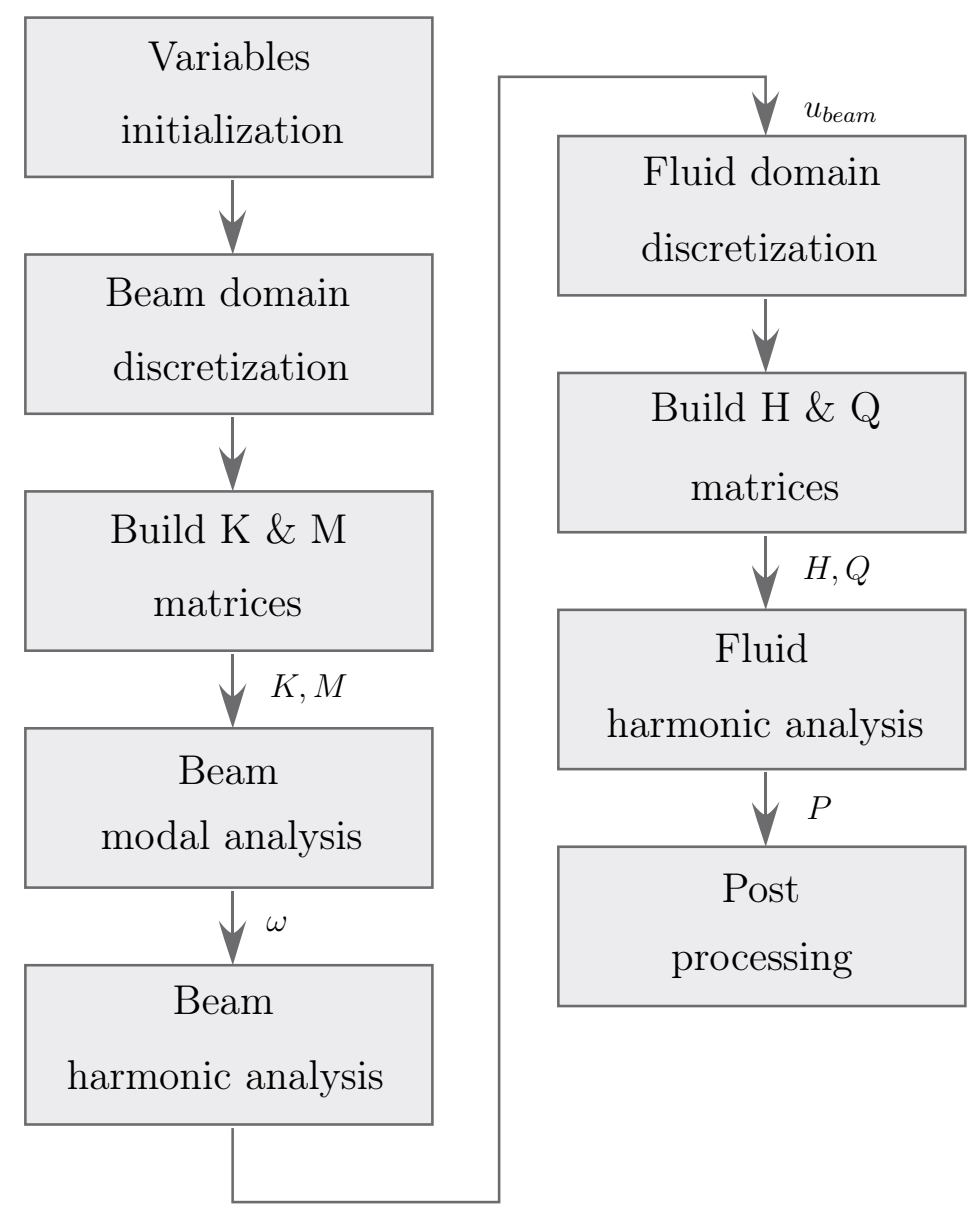

Figure A.2: Steps of a complete analysis of the model fig. 4.9.

\section{a) Beam domain discretization}

The objective of this function is to mesh the beam in order to later build the stiffness and mass matrix of the finite element formulation. The mesh itself is composed of two vectors: one in which the coordinates of each nodes are stored, and the other contain the boundary nodes of each element. This function also make sure that a node is present at the location were the loads are to be applied $\left(\ell_{1}\right.$ and $\ell_{2}$, see fig. $\left.4.1 \mathrm{~b}\right)$ and save the node numbers corresponding to those positions.

\section{b) Build $\mathrm{K}$ and $\mathrm{M}$ matrices}

This function calculates the stiffness and mass matrices of each element using the result presented in 4.1.1 and 4.1.1, and assemble them in the global matrices. Once the matrices are complete they are converted into sparse matrices in order to take less memory. 


\section{c) Beam modal analysis}

This function calculates the frequency for a given mode, using the eigs MatLab function. Since the model only gives the flexural mode, there is no mode inversion and so the desired flexural mode is easily found.

\section{d) Beam harmonic analysis}

Here is calculated the beam response subjected to the two forces at the frequency found in the previous function. No matrix inversion is made, instead the mldivide function of MatLab is used (or also the operator ' $Y$ ').

\section{e) Fluid domain discretization}

This function creates the mesh of the 2D fluid domain and the associated PMLs boundaries. In order to match the displacement nodes of the beam with the $S_{1}$ boundary of the fluid, the coordinate of the $\mathrm{x}$-axis of the fluid between 0 and $\ell$ are the same as the beam. As said before, the reflector is modeled by an unmeshed line. The result produced by this function is shown fig. A.3.

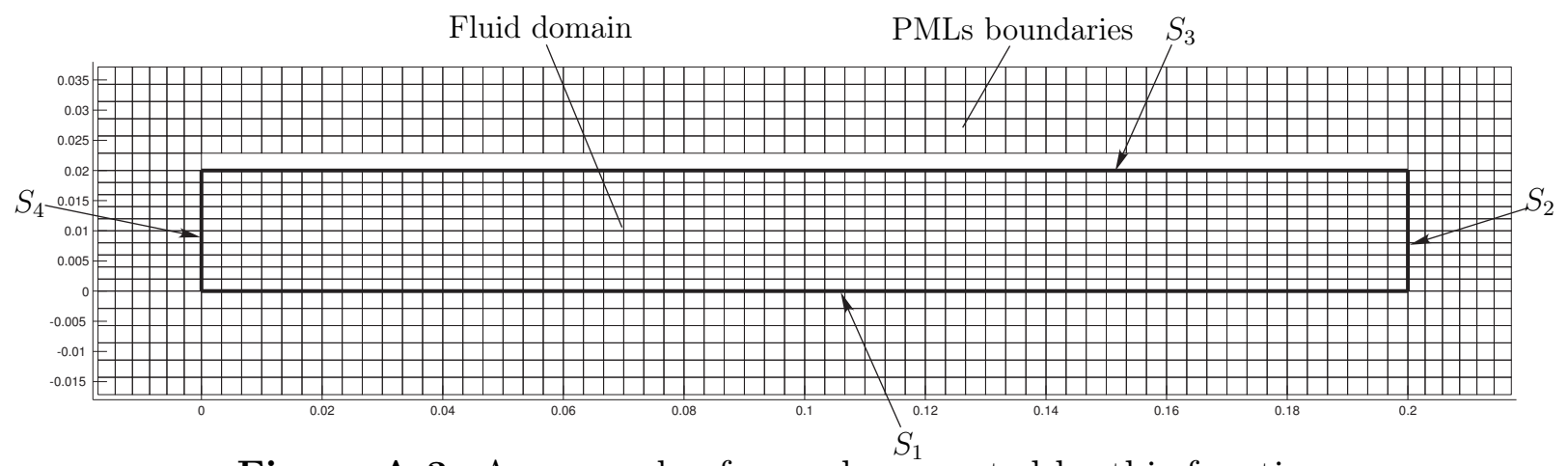

Figure A.3: An example of a mesh generated by this function.

\section{f) Build $\mathrm{H}$ and $\mathrm{Q}$ matrices}

This function calculate both $\mathbf{H}_{\mathbf{e}}$ and $\mathbf{Q}_{\mathbf{e}}$ matrices of the fluid as defined in 4.26 and 4.27, and assemble them in the global matrices $\mathbf{H}$ and $\mathbf{Q}$. Both $\mathbf{H}_{\mathbf{e}}$ and $\mathbf{Q}_{\mathbf{e}}$ are calculated by using a 3x3 Gaussian quadrature rule, and if the element is inside the PMLs domain the matrices are calculated as shown in 4.34. Also, the parfor MatLab function, which executes loop iteration in parallel, is used in order to optimize the speed. The $\mathbf{H}$ and Q matrices are then assembled using sparse matrices, meaning that each values of each matrix is put in a vector, together with two other vector, one has the line position of each value and the other one has the row position of each value. Since most of the matrices 
is full of zeros, using sparse matrices will allow a much smaller size in the memory and a better speed.

\section{g) Fluid harmonic analysis}

This function calculates the harmonic response of the fluid when exposed to the plate vibrations. Since the nodes in the contour $S_{1}$ of the fluid are matched with the nodes of the beam, the vector $F_{1}$ is easily calculated as:

$$
\hat{F}_{1}=\omega^{2} \rho_{0} \hat{V}
$$

where $\hat{V}$ is the vector of the nodal displacements of the beam obtained by the function beam harmonic analysis. The harmonic response of the fluid is then calculated by using eq. 4.29 and the MatLab function mldivide ('Y').

\section{h) Post processing}

The preceding function gives the pressure field, and in order to calculate the Gor'kov levitation potential the particle velocity field is also needed. Both pressure and velocity field are linked by the Euler equation (eq. 2.2, $\rho_{0} \frac{\partial \vec{u}}{\partial t}=-\nabla p$ ). The gradient of pressure can be calculated for each element using $N_{, x}$ and $N_{, y}$ at the center of each element $(\xi, \eta)=$ $(0,0)$, and the value of the pressure at the center of the element is calculated using $N$, as:

$$
\begin{gathered}
\xi=0, \eta=0 \\
\hat{V}_{\text {elem }_{x}}=-\frac{1}{j \omega \rho_{0}} N_{, x} \hat{P}_{e} \\
\hat{V}_{\text {elem }}=-\frac{1}{j \omega \rho_{0}} N_{, y} \hat{P}_{e} \\
\hat{P}_{\text {elem }}=N \hat{P}_{e}
\end{gathered}
$$

Then, the levitation potential is calculated for each element as in eq. 2.3:

$$
U_{\text {elem }}=2 \pi \alpha^{3}\left(\frac{\left\langle P_{\text {elem }}\right\rangle^{2}}{3 \rho_{0} c^{2}} f_{1}-\frac{\rho_{0}\left\langle V_{\text {elem }}\right\rangle^{2}}{2} f_{2}\right)
$$

The patch MatLab function is used to plot the results, and as the values of the levitation potential are given for each elements instead of each nodes, no color interpolation is available. 


\section{A.1.2 PMLs boundaries calibration}

A good calibration of the PMLs boundaries is needed as the final result of the acoustic simulation will heavily depend on it. If the coefficients $\sigma_{x}$ and $\sigma_{y}$ are too low, a partial reflection will occur, or if there are too high the acoustic wave will be reflected. Also, the size of the PMLs and its discretization plays an important role. As an example, a simple acoustic levitation system is modeled as shown in fig. A.4.

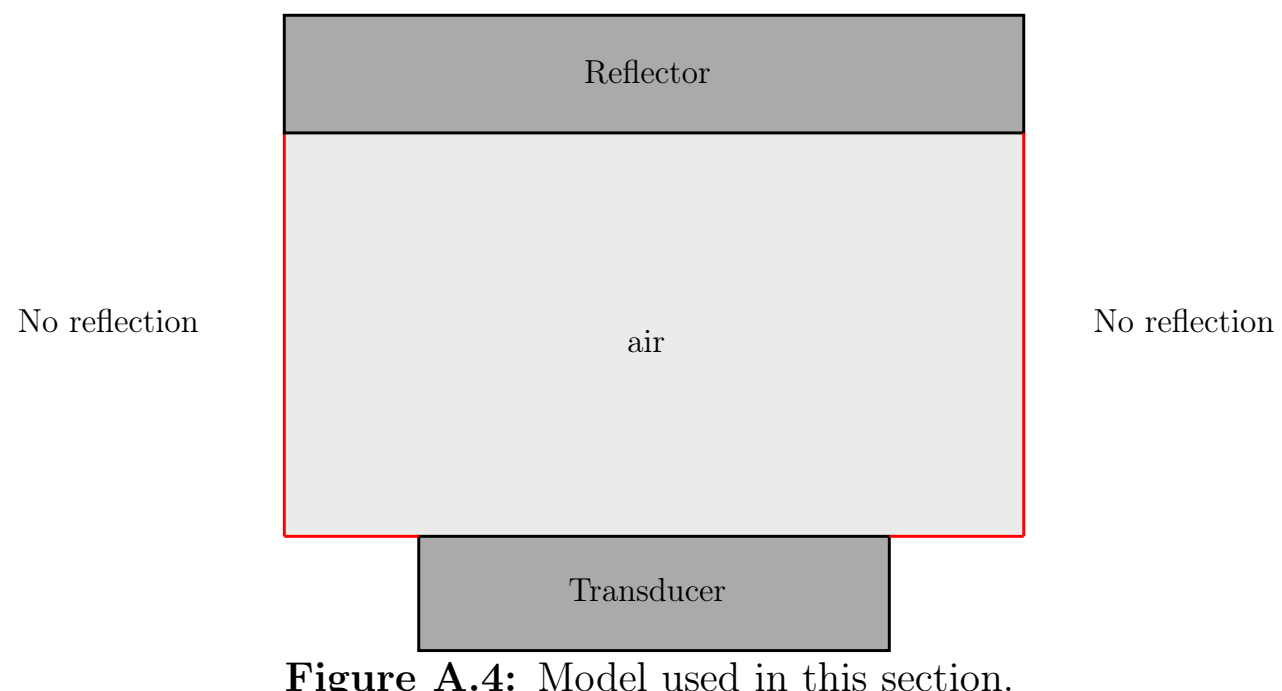

where the transducer is vibrating in a rigid mode. The results of an harmonic analysis with well scaled PMLs boundaries is shown in fig. A.5, the setup presented in fig. A.4 is inside the black box and the PMLs boundaries are outside.

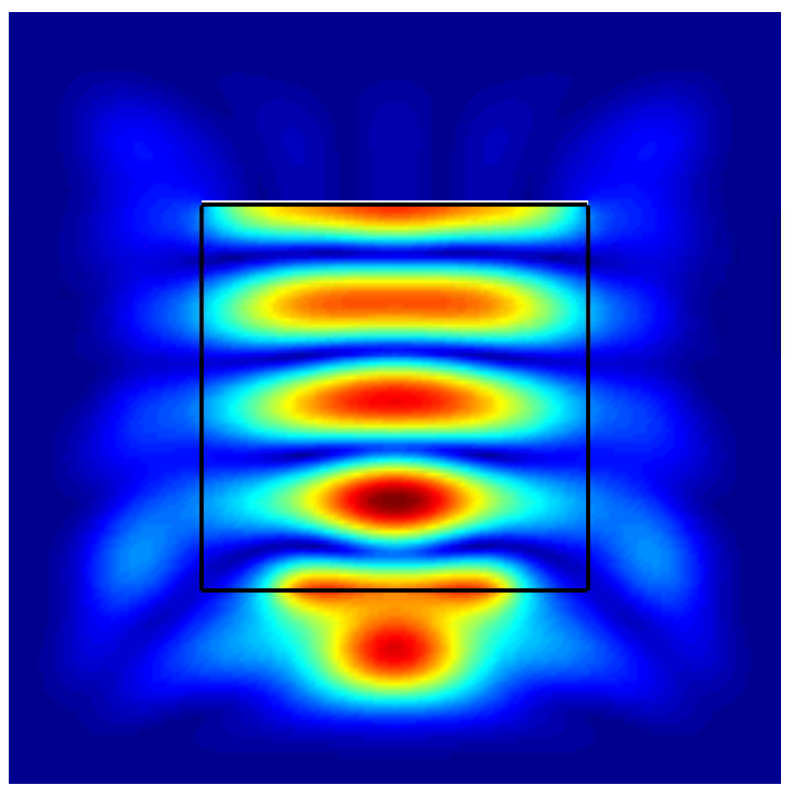

Figure A.5: Normalized pressure amplitude with well scaled PMLs boundaries. 
The figure A.5 shows that when the PMLs boundaries are well scaled, the pressure amplitude inside the PMLs will decrease until being canceled, leaving almost no reflection inside the desired domain.

\section{A.1.3 Analytical comparison of the beam vibration}

The beam model in section 4.1 has, for its modal analysis, analytical solutions. For the free-free Euler-Bernoulli beam model, the analytical solution for the vibration frequency is (GORMAN, 1975):

$$
f=\frac{\beta^{2}}{2 \pi L^{2}} \sqrt{\frac{E I}{\rho A}}
$$

where $L$ is the length of the beam and $\beta$ is the eigenvalue, solution to the following equation: $\sin \beta L \sinh \beta L=0$.

In order to compare both analytical and FEM models, a comparison of the analytical frequency $f_{\text {th }}$ and the frequency given by the FEM $f_{f e m}$ is made for the $6^{t h}, 7^{t h}, 8^{t h}$ and $9^{\text {th }}$ flexural mode. The results is plotted in fig. A.6.

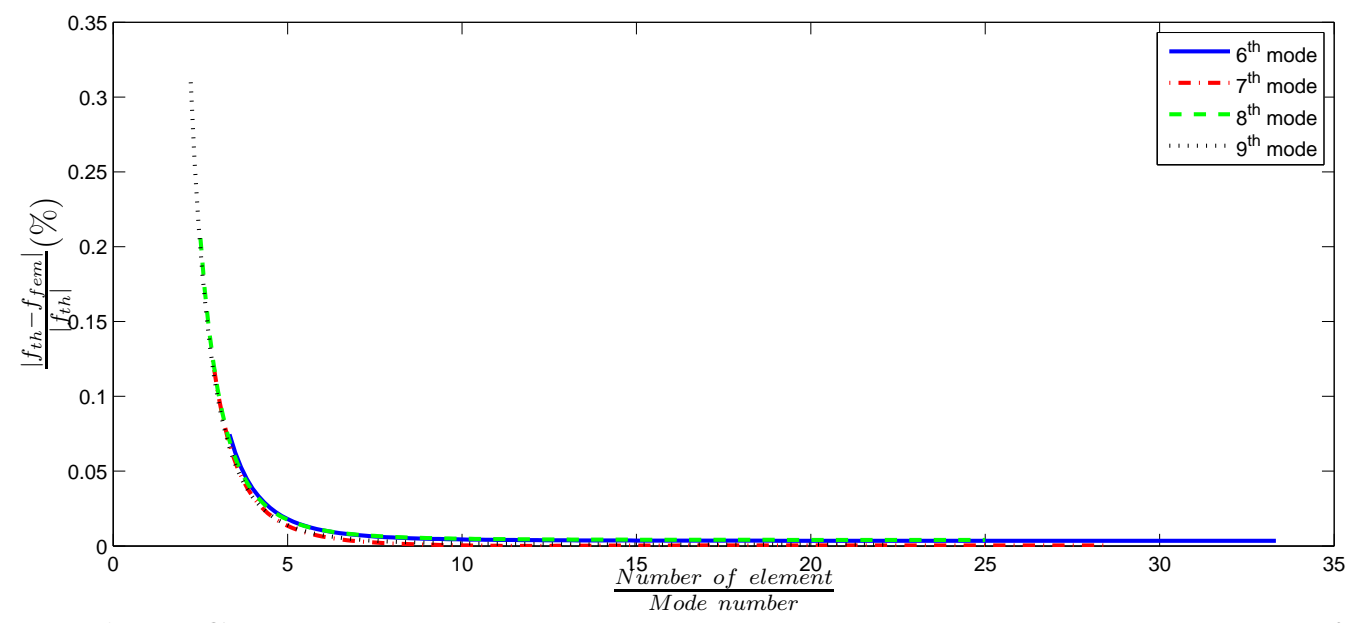

Figure A.6: Comparison between the analytical solution and FEM solution for the frequency

The fig. A.6 shows that the mesh must have at least 10 elements per bending in order to have a good consistency between the analytical and FEM results. 


\section{Appendix B}

\section{B.1 Hamilton's principle applied to the Timoshenko straight beam theory}

The kinetic energy $\mathcal{T}_{u_{s}}$ due to the horizontal motion of the beam is:

$$
\mathcal{T}_{u_{s}}=\frac{1}{2} \int_{0}^{L} \rho A\left(\frac{\partial u_{s}}{\partial t}\right)^{2} \mathrm{~d} x
$$

The kinetic energy $\mathcal{T}_{v_{s}}$ due to the vertical motion of the beam is:

$$
\mathcal{T}_{v_{s}}=\frac{1}{2} \int_{0}^{L} \rho A\left(\frac{\partial v_{s}}{\partial t}\right)^{2} \mathrm{~d} x
$$

The kinetic energy $\mathcal{T}_{\varphi_{s}}$ due to the rotational inertia of the beam is:

$$
\mathcal{T}_{\varphi_{s}}=\frac{1}{2} \int_{0}^{L} \rho I\left(\frac{\partial \varphi_{s}}{\partial t}\right)^{2} \mathrm{~d} x
$$

The deformation energy $\mathcal{V}_{\varepsilon_{s}}$ due to the normal strain of the beam is:

$$
\mathcal{V}_{\varepsilon_{s}}=\frac{1}{2} \int_{0}^{L} E A\left(\frac{\partial u_{s}}{\partial x}\right)^{2} \mathrm{~d} x
$$

The deformation energy $\mathcal{V}_{b_{s}}$ due to the bending moment of the beam is:

$$
\mathcal{V}_{b_{s}}=\frac{1}{2} \int_{0}^{L} E I\left(\frac{\partial \varphi_{s}}{\partial x}\right)^{2} \mathrm{~d} x
$$

The deformation energy $\mathcal{V}_{Q_{s}}$ due to the shear deformation of the beam is:

$$
\mathcal{V}_{Q_{s}}=\frac{1}{2} \int_{0}^{L} k G A\left(\frac{\partial v_{s}}{\partial x}-\varphi_{s}\right)^{2} \mathrm{~d} x
$$

The Euler-Lagrange equation of the problem is: 


$$
\mathcal{L}_{s}=\mathcal{T}_{u_{s}}+\mathcal{T}_{v_{s}}+\mathcal{T}_{\varphi_{s}}-\mathcal{V}_{\varepsilon_{s}}-\mathcal{V}_{b_{s}}-\mathcal{V}_{Q_{s}}
$$

Applying the Hamilton's principle $\delta \int_{t_{1}}^{t_{2}} \mathcal{L}_{s} \mathrm{~d} t=0$ :

$\delta \int_{t_{1}}^{t_{2}} \mathcal{L}_{s} \mathrm{~d} t=\delta \int_{t_{1}}^{t_{2}} \mathcal{T}_{u_{s}} \mathrm{~d} t+\delta \int_{t_{1}}^{t_{2}} \mathcal{T}_{s_{s}} \mathrm{~d} t+\delta \int_{t_{1}}^{t_{2}} \mathcal{T}_{\varphi_{s}} \mathrm{~d} t-\delta \int_{t_{1}}^{t_{2}} \mathcal{V}_{\mathcal{E}_{s}} \mathrm{~d} t-\delta \int_{t_{1}}^{t_{2}} \mathcal{V}_{b_{s}} \mathrm{~d} t-\delta \int_{t_{1}}^{t_{2}} \mathcal{V}_{Q_{s}} \mathrm{~d} t$ $=0$

where

$$
\begin{aligned}
\delta \int_{t_{1}}^{t_{2}} \mathcal{T}_{u_{s}} \mathrm{~d} t & =\int_{t_{1}}^{t_{2}} \int_{0}^{L} \rho A \frac{\partial u_{s}}{\partial t} \delta\left(\frac{\partial u_{s}}{\partial t}\right) \mathrm{d} x \mathrm{~d} t \\
& =\left[\int_{0}^{L} \rho A \frac{\partial u_{s}}{\partial t} \delta u_{s} \mathrm{~d} x\right]_{t_{1}}^{t_{2}}-\int_{t_{1}}^{t_{2}} \int_{0}^{L} \rho A \frac{\partial^{2} u_{s}}{\partial t^{2}} \delta u_{s} \mathrm{~d} x \mathrm{~d} t
\end{aligned}
$$

since $\left.\delta u_{s}\right|_{t_{1}} ^{t_{2}}=0$ :

$$
\delta \int_{t_{1}}^{t_{2}} \mathcal{T}_{u_{s}} \mathrm{~d} t=-\int_{t_{1}}^{t_{2}} \int_{0}^{L} \rho A \frac{\partial^{2} u_{s}}{\partial t^{2}} \delta u_{s} \mathrm{~d} x \mathrm{~d} t
$$

similarly:

$$
\begin{gathered}
\delta \int_{t_{1}}^{t_{2}} \mathcal{T}_{v_{s}} \mathrm{~d} t=-\int_{t_{1}}^{t_{2}} \int_{0}^{L} \rho A \frac{\partial^{2} v_{s}}{\partial t^{2}} \delta v_{s} \mathrm{~d} x \mathrm{~d} t \\
\delta \int_{t_{1}}^{t_{2}} \mathcal{T}_{\varphi_{s}} \mathrm{~d} t=-\int_{t_{1}}^{t_{2}} \int_{0}^{L} \rho I \frac{\partial^{2} \varphi_{s}}{\partial t^{2}} \delta \varphi_{s} \mathrm{~d} x \mathrm{~d} t \\
\delta \int_{t_{1}}^{t_{2}} \mathcal{V}_{\varepsilon_{s}} \mathrm{~d} t=\int_{t_{1}}^{t_{2}} \int_{0}^{L} E A \frac{\partial u_{s}}{\partial x} \delta\left(\frac{\partial u_{s}}{\partial x}\right) \mathrm{d} x \mathrm{~d} t \\
=\left[\int_{t_{1}}^{t_{2}} E A \frac{\partial u_{s}}{\partial x} \delta u_{s} \mathrm{~d} t\right]_{0}^{L}-\int_{t_{1}}^{t_{2}} \int_{0}^{L} E A \frac{\partial^{2} u_{s}}{\partial x^{2}} \delta u_{s} \mathrm{~d} x \mathrm{~d} t
\end{gathered}
$$

since $\left.\delta u_{s}\right|_{0} ^{L}=0$ :

$$
\delta \int_{t_{1}}^{t_{2}} \mathcal{V}_{\varepsilon_{s}} \mathrm{~d} t=-\int_{t_{1}}^{t_{2}} \int_{0}^{L} E A \frac{\partial^{2} u_{s}}{\partial x^{2}} \delta u_{s} \mathrm{~d} x \mathrm{~d} t
$$

similarly:

$$
\delta \int_{t_{1}}^{t_{2}} \mathcal{V}_{b_{s}} \mathrm{~d} t=-\int_{t_{1}}^{t_{2}} \int_{0}^{L} E I \frac{\partial^{2} \varphi_{s}}{\partial x^{2}} \delta \varphi_{s} \mathrm{~d} x \mathrm{~d} t
$$




$$
\begin{aligned}
\delta \int_{t_{1}}^{t_{2}} \mathcal{V}_{Q_{s}} \mathrm{~d} t & =\int_{t_{1}}^{t_{2}} \int_{0}^{L} k G A\left(\frac{\partial v_{s}}{\partial x}-\varphi_{s}\right) \delta\left(\frac{\partial v_{s}}{\partial x}-\varphi_{s}\right) \mathrm{d} x \mathrm{~d} t \\
& =\int_{t_{1}}^{t_{2}} \int_{0}^{L} k G A\left[\left(\frac{\partial v_{s}}{\partial x}-\varphi_{s}\right) \delta\left(\frac{\partial v_{s}}{\partial x}\right)-\left(\frac{\partial v_{s}}{\partial x}-\varphi_{s}\right) \delta \varphi_{s}\right] \mathrm{d} x \mathrm{~d} t \\
\delta \int_{t_{1}}^{t_{2}} \mathcal{V}_{Q_{s}} \mathrm{~d} t & =-\int_{t_{1}}^{t_{2}} \int_{0}^{L} k G A\left[\left(\frac{\partial^{2} v_{s}}{\partial x^{2}}-\frac{\partial \varphi_{s}}{\partial x}\right) \delta v_{s}+\left(\frac{\partial v_{s}}{\partial x}-\varphi_{s}\right) \delta \varphi_{s}\right] \mathrm{d} x \mathrm{~d} t
\end{aligned}
$$

which yield:

$$
\begin{gathered}
\rho A \frac{\mathrm{d}^{2} u_{s}}{\mathrm{~d} t^{2}}-E A \frac{\partial^{2} u_{s}}{\partial x^{2}}=0 \\
\rho A \frac{\mathrm{d}^{2} v_{s}}{\mathrm{~d} t^{2}}-k G A\left(\frac{\partial^{2} v_{s}}{\partial x^{2}}-\frac{\partial \varphi}{\partial x}\right)=0 \\
\rho I \frac{\mathrm{d}^{2} \varphi_{s}}{\mathrm{~d} t^{2}}-E I \frac{\partial^{2} \varphi_{s}}{\partial t^{2}}-k G A\left(\frac{\partial v_{s}}{\partial x}-\varphi\right)=0
\end{gathered}
$$

\section{B.2 Hamilton's principle applied to the Timoshenko curved beam theory}

The kinetic energy $\mathcal{T}_{u_{c}}$ due to the horizontal motion of the beam is:

$$
\mathcal{T}_{u_{c}}=\frac{1}{2} \int_{-\frac{\pi}{2}}^{\frac{\pi}{2}} \rho A R\left(\frac{\partial u_{c}}{\partial t}\right)^{2} \mathrm{~d} \theta
$$

The kinetic energy $\mathcal{T}_{v_{c}}$ due to the vertical motion of the beam is:

$$
\begin{aligned}
\mathcal{T}_{v_{c}} & =\frac{1}{2} \int_{-\frac{\pi}{2}}^{\frac{\pi}{2}} \rho A R\left(\frac{\partial v_{c}}{\partial t}\right)^{2} \mathrm{~d} \theta \\
& =\frac{1}{2} \int_{-\frac{\pi}{2}}^{\frac{\pi}{2}} \rho A R\left(\frac{\partial}{\partial t} \frac{\partial u_{c}}{\partial \theta}\right)^{2} \mathrm{~d} \theta
\end{aligned}
$$

The kinetic energy $\mathcal{T}_{\varphi_{c}}$ due to the rotational inertia of the beam is:

$$
\mathcal{T}_{\varphi_{c}}=\frac{1}{2} \int_{-\frac{\pi}{2}}^{\frac{\pi}{2}} \rho I R\left(\frac{\partial \varphi_{c}}{\partial t}\right)^{2} \mathrm{~d} \theta
$$

The deformation energy $\mathcal{V}_{b_{c}}$ due to the bending moment of the beam is: 


$$
\mathcal{V}_{b_{c}}=\frac{1}{2} \int_{-\frac{\pi}{2}}^{\frac{\pi}{2}} \frac{E I}{R}\left(\frac{\partial \varphi_{c}}{\partial \theta}\right)^{2} \mathrm{~d} \theta
$$

The deformation energy $\mathcal{V}_{Q_{c}}$ due to the shear deformation of the beam is:

$$
\begin{aligned}
\mathcal{V}_{Q_{c}} & =\frac{1}{2} \int_{-\frac{\pi}{2}}^{\frac{\pi}{2}} \frac{k A G}{R}\left(\frac{\partial v_{c}}{\partial \theta}+u_{c}-R \varphi_{c}\right)^{2} \mathrm{~d} \theta \\
& =\frac{1}{2} \int_{-\frac{\pi}{2}}^{\frac{\pi}{2}} \frac{k A G}{R}\left(\frac{\partial^{2} u_{c}}{\partial \theta^{2}}+u_{c}-R \varphi_{c}\right)^{2} \mathrm{~d} \theta
\end{aligned}
$$

The Euler-Lagrange equation of the problem is:

$$
\mathcal{L}_{c}=\mathcal{T}_{u_{c}}+\mathcal{T}_{v_{c}}+\mathcal{T}_{\varphi_{c}}-\mathcal{V}_{b_{c}}-\mathcal{V}_{Q_{c}}
$$

Applying the Hamilton's principle $\delta \int_{t_{1}}^{t_{2}} \mathcal{L}_{c} \mathrm{~d} t=0$ :

$$
\begin{aligned}
\delta \int_{t_{1}}^{t_{2}} \mathcal{L}_{c} \mathrm{~d} t & =\delta \int_{t_{1}}^{t_{2}} \mathcal{T}_{u_{c}} \mathrm{~d} t+\delta \int_{t_{1}}^{t_{2}} \mathcal{T}_{v_{c}} \mathrm{~d} t+\delta \int_{t_{1}}^{t_{2}} \mathcal{T}_{\varphi_{c}} \mathrm{~d} t-\delta \int_{t_{1}}^{t_{2}} \mathcal{V}_{b_{c}} \mathrm{~d} t-\delta \int_{t_{1}}^{t_{2}} \mathcal{V}_{Q_{c}} \mathrm{~d} t \\
& =0
\end{aligned}
$$

where

$$
\begin{aligned}
\delta \int_{t_{1}}^{t_{2}} \mathcal{T}_{u_{c}} \mathrm{~d} t & =\int_{t_{1}}^{t_{2}} \int_{-\frac{\pi}{2}}^{\frac{\pi}{2}} \rho A R \frac{\partial u_{c}}{\partial t} \delta\left(\frac{\partial u_{c}}{\partial t}\right) \mathrm{d} \theta \mathrm{d} t \\
& =\left[\int_{-\frac{\pi}{2}}^{\frac{\pi}{2}} \rho A R \frac{\partial u_{c}}{\partial t} \delta u_{c} \mathrm{~d} \theta\right]_{t_{1}}^{t_{2}}-\int_{t_{1}}^{t_{2}} \int_{-\frac{\pi}{2}}^{\frac{\pi}{2}} \rho A \frac{\partial^{2} u_{c}}{\partial t^{2}} \delta u_{c} \mathrm{~d} \theta \mathrm{d} t
\end{aligned}
$$

since $\left.\delta u_{c}\right|_{t_{1}} ^{t_{2}}=0$ :

$$
\delta \int_{t_{1}}^{t_{2}} \mathcal{T}_{u_{c}} \mathrm{~d} t=-\int_{t_{1}}^{t_{2}} \int_{-\frac{\pi}{2}}^{\frac{\pi}{2}} \rho A R \frac{\partial^{2} u_{c}}{\partial t^{2}} \delta u_{c} \mathrm{~d} \theta \mathrm{d} t
$$

similarly:

$$
\begin{gathered}
\delta \int_{t_{1}}^{t_{2}} \mathcal{T}_{v_{c}} \mathrm{~d} t=\int_{t_{1}}^{t_{2}} \int_{-\frac{\pi}{2}}^{\frac{\pi}{2}} \rho A R \frac{\partial^{2}}{\partial t^{2}} \frac{\partial^{2} u_{c}}{\partial \theta^{2}} \delta u_{c} \mathrm{~d} \theta \mathrm{d} t \\
\delta \int_{t_{1}}^{t_{2}} \mathcal{T}_{\varphi_{c}} \mathrm{~d} t=-\int_{t_{1}}^{t_{2}} \int_{-\frac{\pi}{2}}^{\frac{\pi}{2}} \rho A R \frac{\partial^{2} \varphi_{c}}{\partial t^{2}} \delta \varphi_{c} \mathrm{~d} \theta \mathrm{d} t
\end{gathered}
$$




$$
\begin{aligned}
\delta \int_{t_{1}}^{t_{2}} \mathcal{V}_{b_{c}} \mathrm{~d} t & =\frac{1}{2} \int_{-\frac{\pi}{2}}^{\frac{\pi}{2}} \frac{E I}{R} \frac{\partial \varphi_{c}}{\partial \theta} \delta\left(\frac{\partial \varphi_{c}}{\partial \theta}\right) \mathrm{d} \theta \\
& =\left[\int_{t_{1}}^{t_{2}} \frac{E I}{R} \frac{\partial \varphi_{c}}{\partial \theta} \delta \varphi_{c} \mathrm{~d} t\right]_{-\frac{\pi}{2}}^{\frac{\pi}{2}}-\int_{t_{1}}^{t_{2}} \int_{-\frac{\pi}{2}}^{\frac{\pi}{2}} \frac{E I}{R} \frac{\partial^{2} \varphi_{c}}{\partial t^{2}} \delta \varphi_{c} \mathrm{~d} \theta \mathrm{d} t
\end{aligned}
$$

since $\left.\delta \varphi_{c}\right|_{-\frac{\pi}{2}} ^{\frac{\pi}{2}}=0$

$$
\delta \int_{t_{1}}^{t_{2}} \mathcal{V}_{b_{c}} \mathrm{~d} t=-\int_{t_{1}}^{t_{2}} \int_{-\frac{\pi}{2}}^{\frac{\pi}{2}} \frac{E I}{R} \frac{\partial^{2} \varphi_{c}}{\partial t^{2}} \delta \varphi_{c} \mathrm{~d} \theta \mathrm{d} t
$$

similarly:

$$
\begin{aligned}
\delta \int_{t_{1}}^{t_{2}} \mathcal{V}_{Q_{c}} \mathrm{~d} t & =\int_{t_{1}}^{t_{2}} \int_{-\frac{\pi}{2}}^{\frac{\pi}{2}} \frac{k A G}{R}\left[\left(\frac{\partial^{4} u_{c}}{\partial \theta^{4}}+2 \frac{\partial^{2} u_{c}}{\partial \theta^{2}}+u_{c}-R \frac{\partial^{2} \varphi_{c}}{\partial \theta^{2}}-R \varphi_{c}\right) \delta u_{c}\right. \\
& \left.-R\left(\frac{\partial^{2} u_{c}}{\partial \theta^{2}}+u_{c}-R \varphi_{c}\right) \delta \varphi_{c}\right] \mathrm{d} \theta \mathrm{d} t
\end{aligned}
$$

which yield:

$$
\begin{gathered}
\frac{\rho R^{2}}{k G}\left[\frac{\partial^{2} u_{c}}{\partial t^{2}}-\frac{\partial^{2}}{\partial t^{2}} \frac{\partial^{2} u_{c}}{\partial \theta^{2}}\right]+\frac{\partial^{2} u_{c}}{\partial \theta^{4}}+2 \frac{\partial^{2} u_{c}}{\partial \theta^{2}}+u_{c}-R \varphi-R \frac{\partial^{2} \varphi}{\partial \theta^{2}}=0 \\
\frac{\rho I R}{k A G} \frac{\partial^{2} \varphi}{\partial t^{2}}-\frac{E I}{k A G R} \frac{\partial^{2} \varphi}{\partial \theta^{2}}+R \varphi-\frac{\partial^{2} u_{c}}{\partial \theta^{2}}-u_{c}=0
\end{gathered}
$$

\section{B.3 MatLab program for iterative design with the analytical model of the ring-type vibrator}

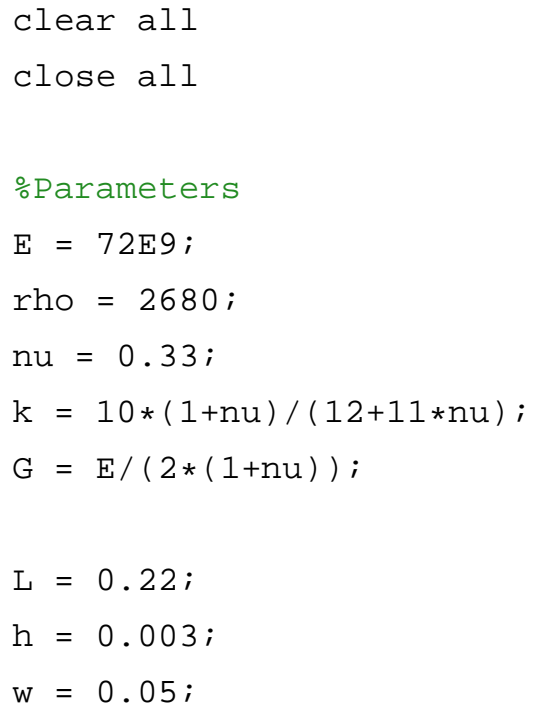




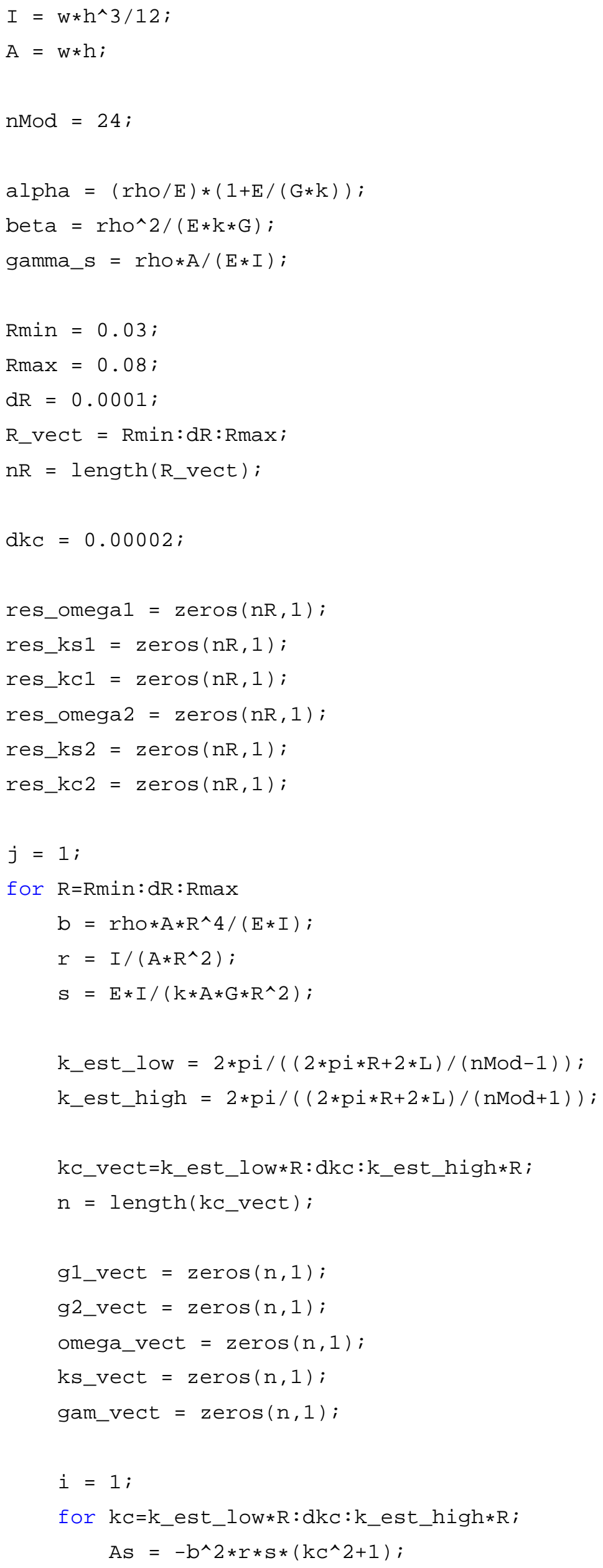




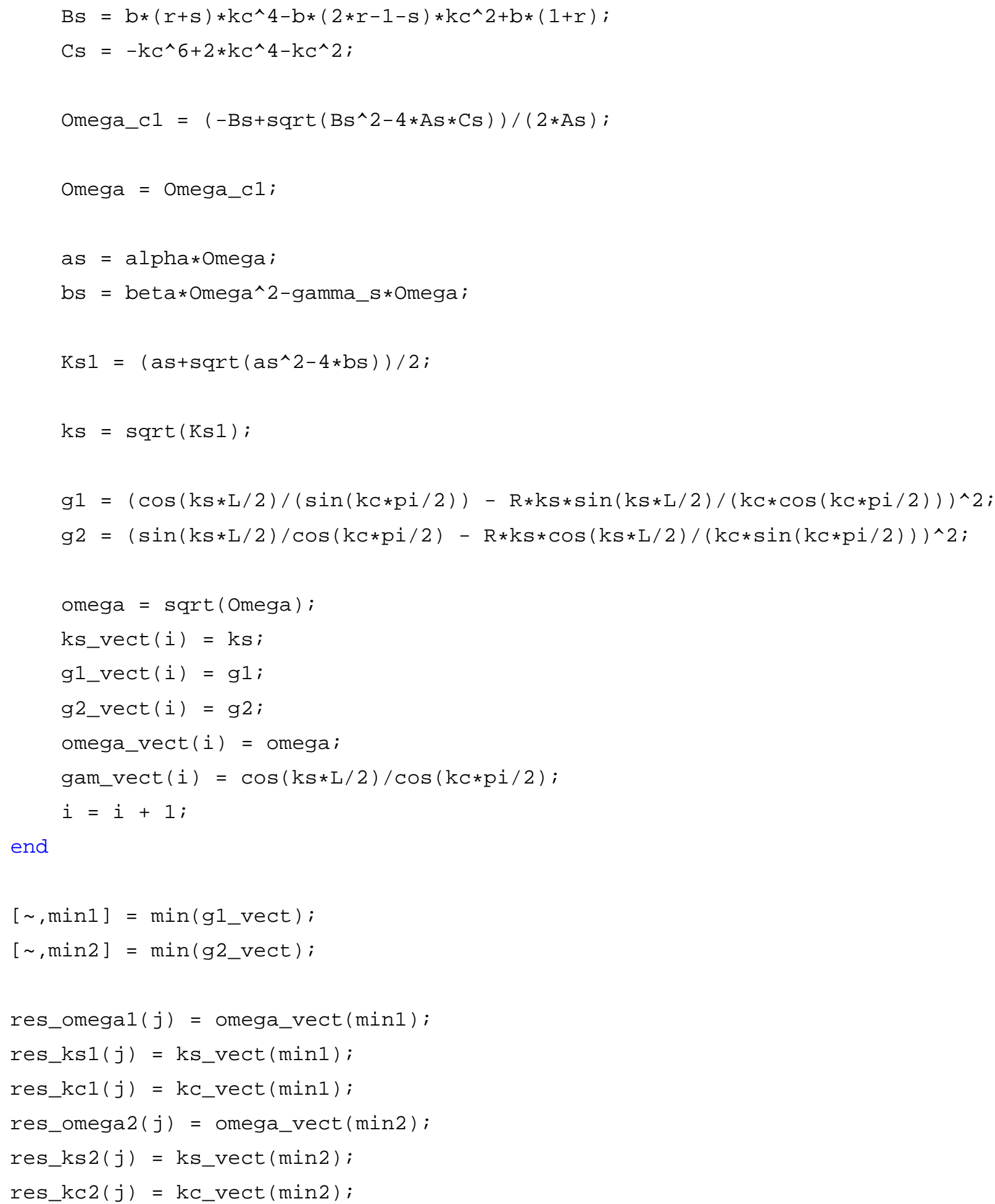


set (p2,'Color', 'r');

set (p3, 'Linestyle', '--');

legend([p1 p2 p3], 'Solution 1', 'Solution 2', '|\Deltaf|/f');

xlabel ('R (m)')

ylabel (ax (1), 'Frequency (Hz)')

ylabel (ax (2), 'Frequency difference (\%)') 


\section{Appendix C}

\section{C.1 Script for acoustic levitation transportation}

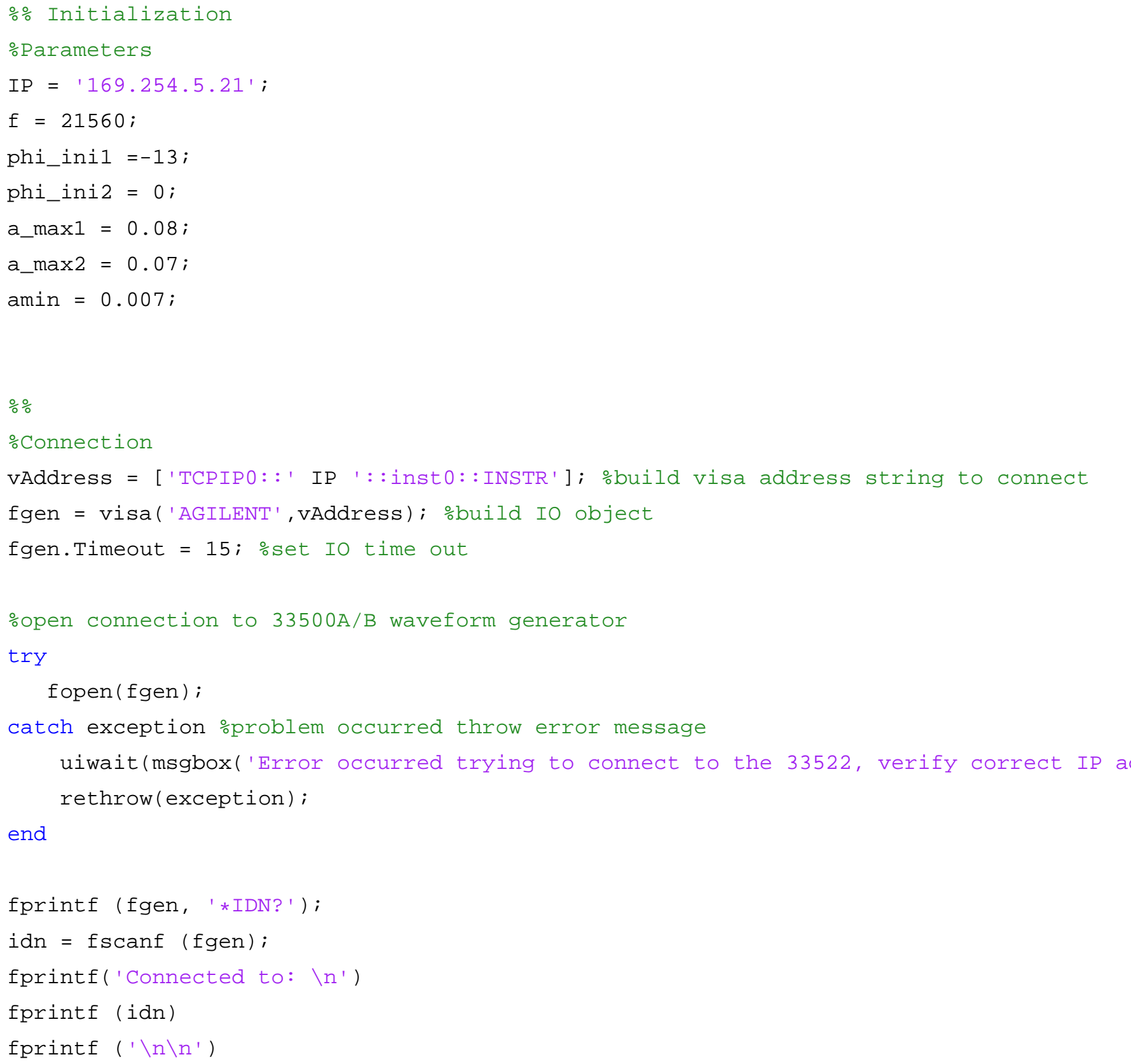




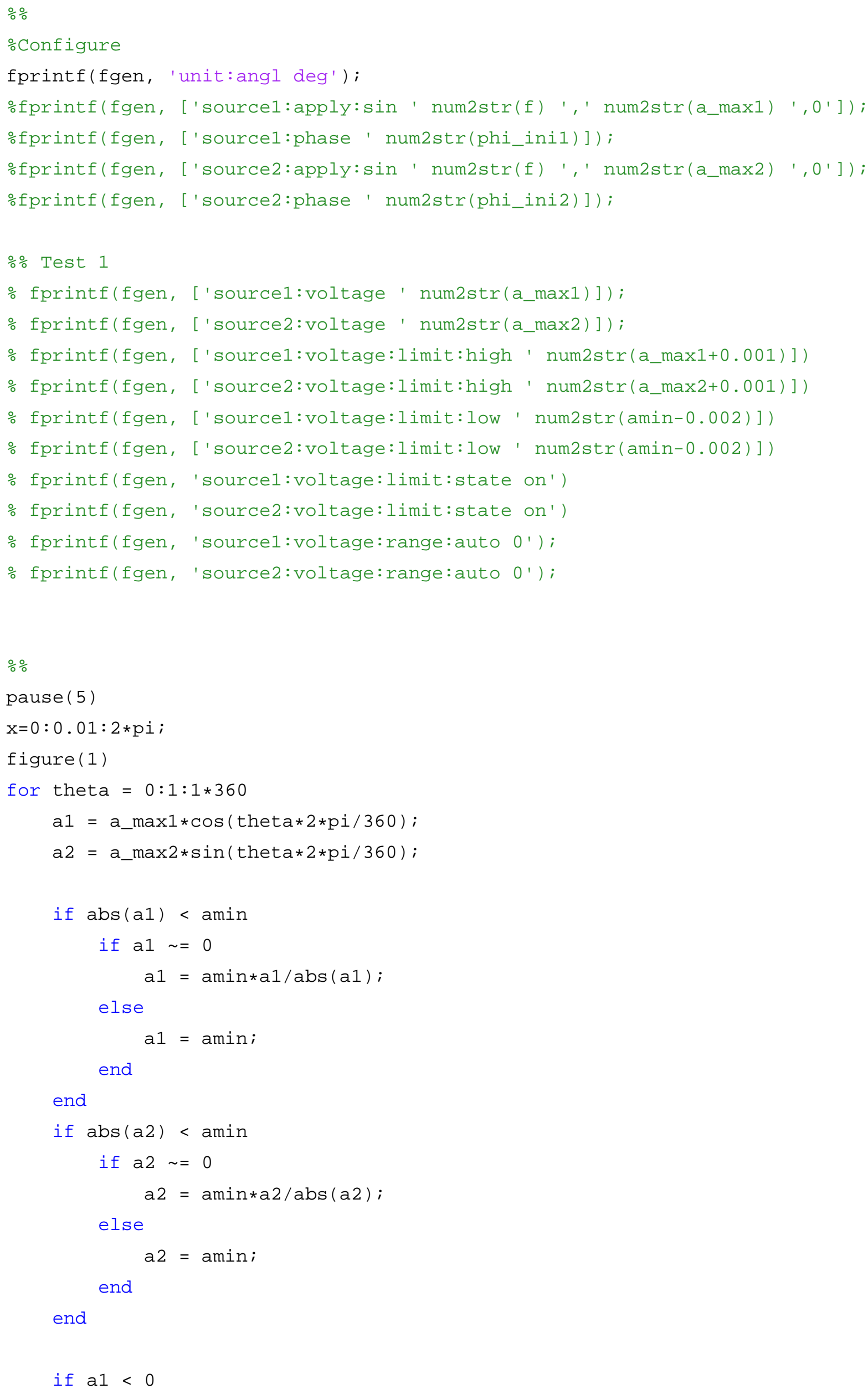


phil = phi_ini1+180;

ophi2 = phi_ini2+180;

$\mathrm{a} 1=\mathrm{abs}(\mathrm{a} 1)$;

else

ㅇpi2 = phi_ini2;

phil = phi_inil;

end

if $a 2<0$

phi2 = phi_ini2+180;

$\circ$ phi1 = phi_ini1-180;

$\mathrm{a} 2=\mathrm{abs}(\mathrm{a} 2)$;

else

phi2 = phi_ini2;

ㅇpil = phi_inil;

end

fprintf(fgen, ['source1:voltage ' num2str(a1)]);

fprintf(fgen, ['source1:phase ' num2str(phi1)]);

fprintf(fgen, ['source2:voltage ' num2str(a2)]);

fprintf(fgen, ['source2:phase ' num2str(phi2)]);

pause $(0.001)$

a1

a2

phi1

phi2

$\operatorname{plot}(x, \cos (x+p h i 1) * a 1+\sin (x+p h i 2) * a 2)$

end

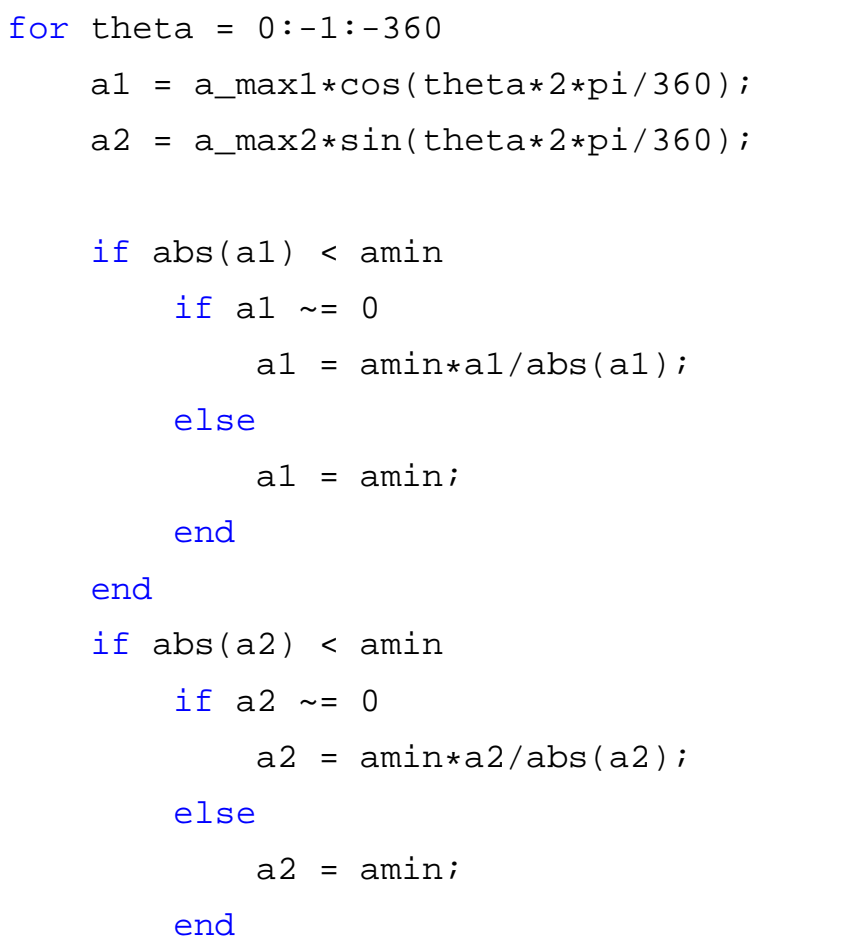


end

if $a 1<0$

phil = phi_ini1+180;

ophi2 = phi_ini2+180;

$\mathrm{a} 1=\operatorname{abs}(\mathrm{a} 1)$;

else

$\frac{\circ}{\circ}$ phi2 = phi_ini2;

phil = phi_inil;

end

if $a 2<0$

phi2 = phi_ini2+180;

phil = phi_ini1-180;

$\mathrm{a} 2$ = abs (a2);

else

phi2 = phi_ini2;

ㅇphil = phi_inil;

end

fprintf(fgen, ['source1:voltage ' num2str(a1)]);

fprintf(fgen, ['source1:phase ' num2str(phi1)]);

fprintf(fgen, ['source2:voltage ' num2str(a2)]);

fprintf(fgen, ['source2:phase ' num2str(phi2)]);

pause $(0.2)$

a1

a2

phi1

phi2

plot $(x, \cos (x+p h i 1) * a 1+\sin (x+p h i 2) * a 2)$

end

$\div \circ$

○Read Error

fprintf(fgen, 'SYST:ERR?');

errorstr $=$ fscanf (fgen);

o error checking

if strncmp (errorstr, '+0,"No error"',13)

errorcheck = 'Arbitrary waveform generated without any error $\backslash \mathrm{n}$ ';

fprintf (errorcheck)

else

errorcheck = ['Error reported: ', errorstr];

fprintf (errorcheck)

end

fclose (fgen); 


\section{C.2 Script for vibration measurements}

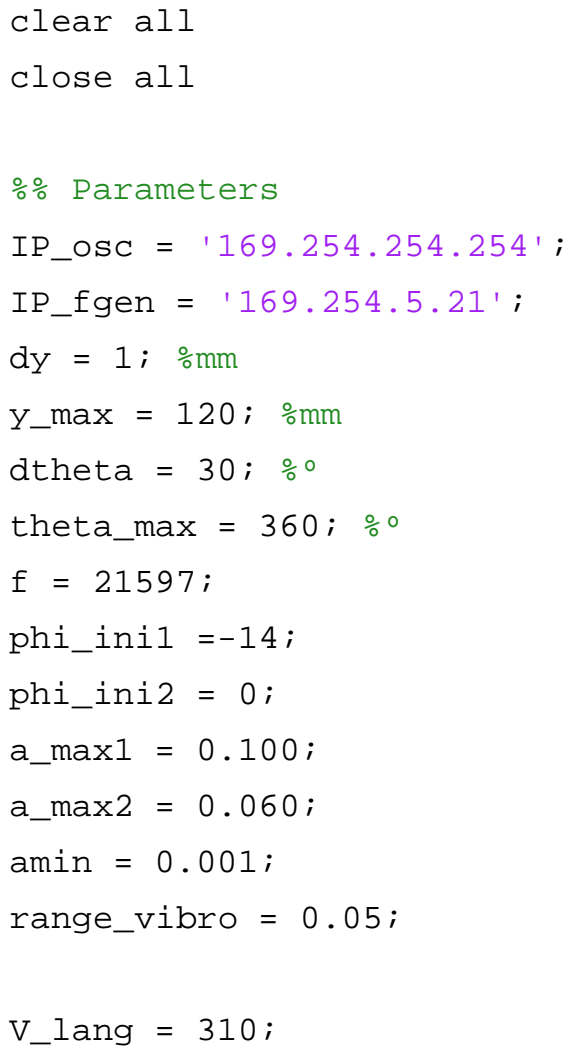




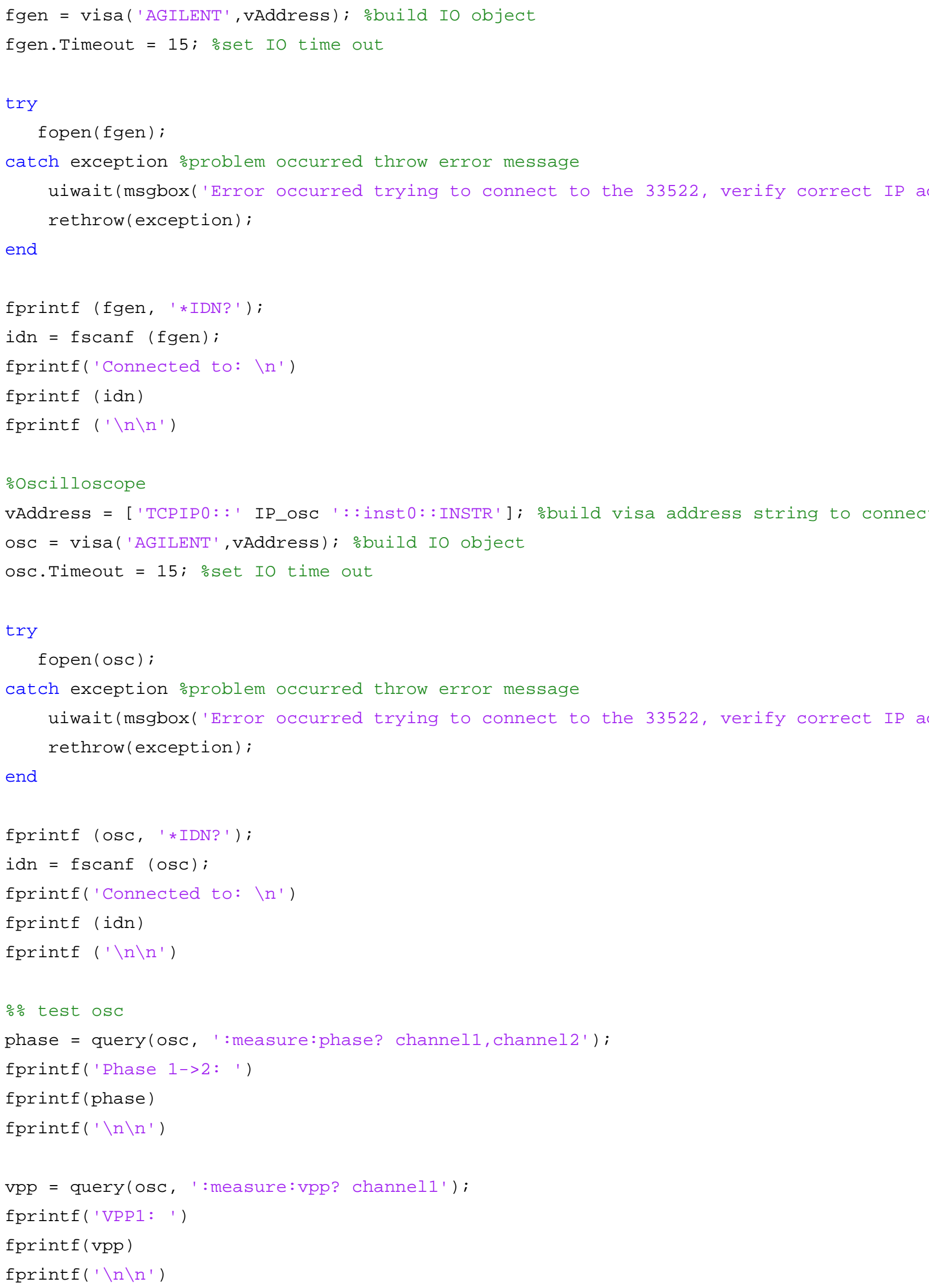




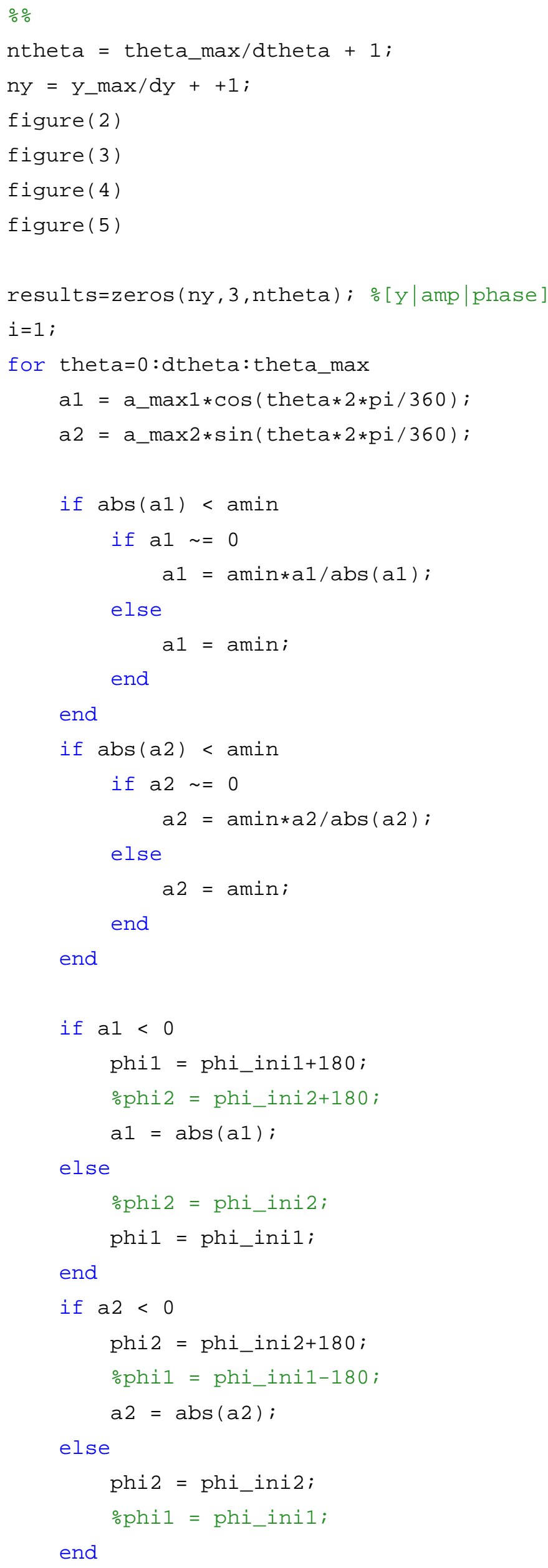




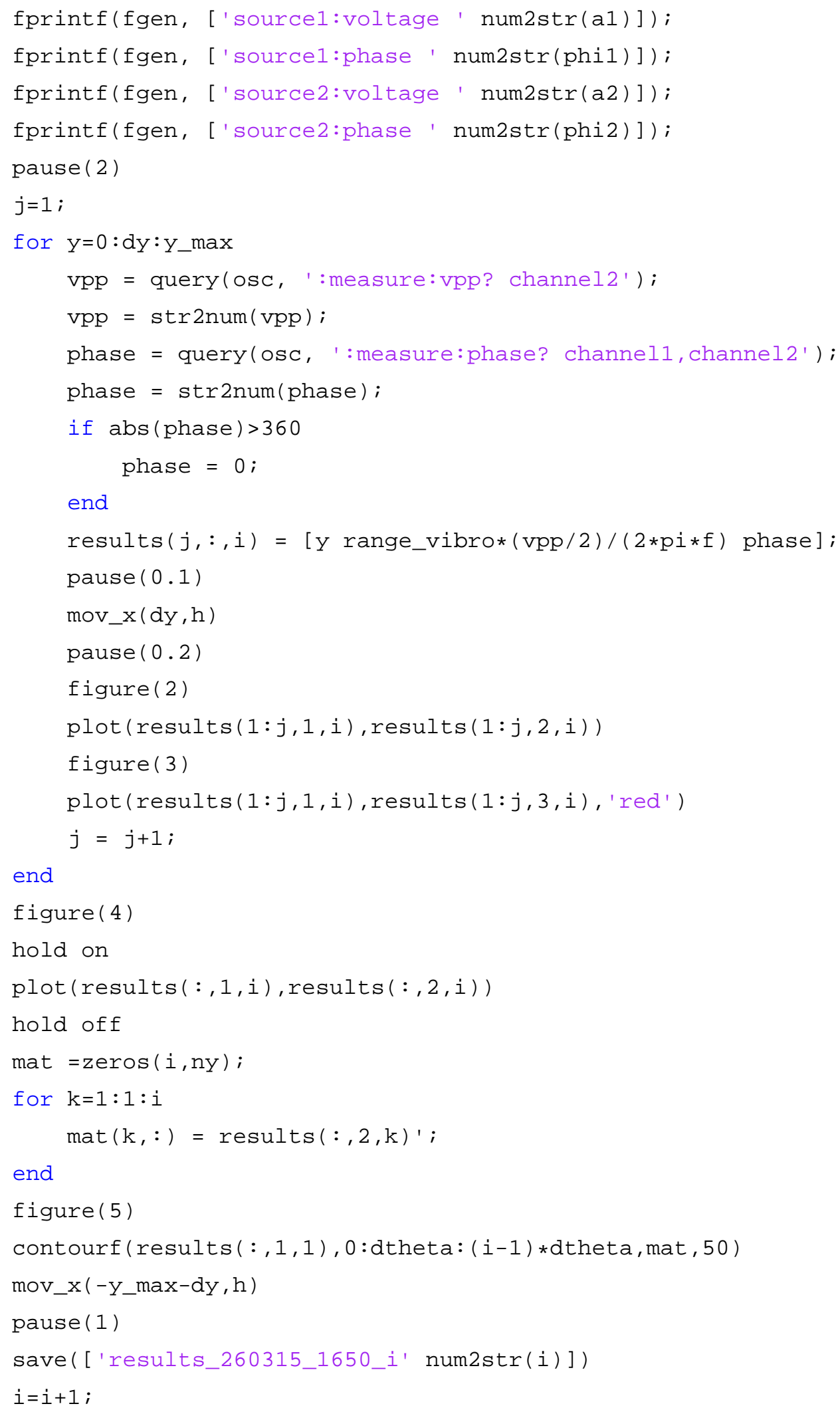




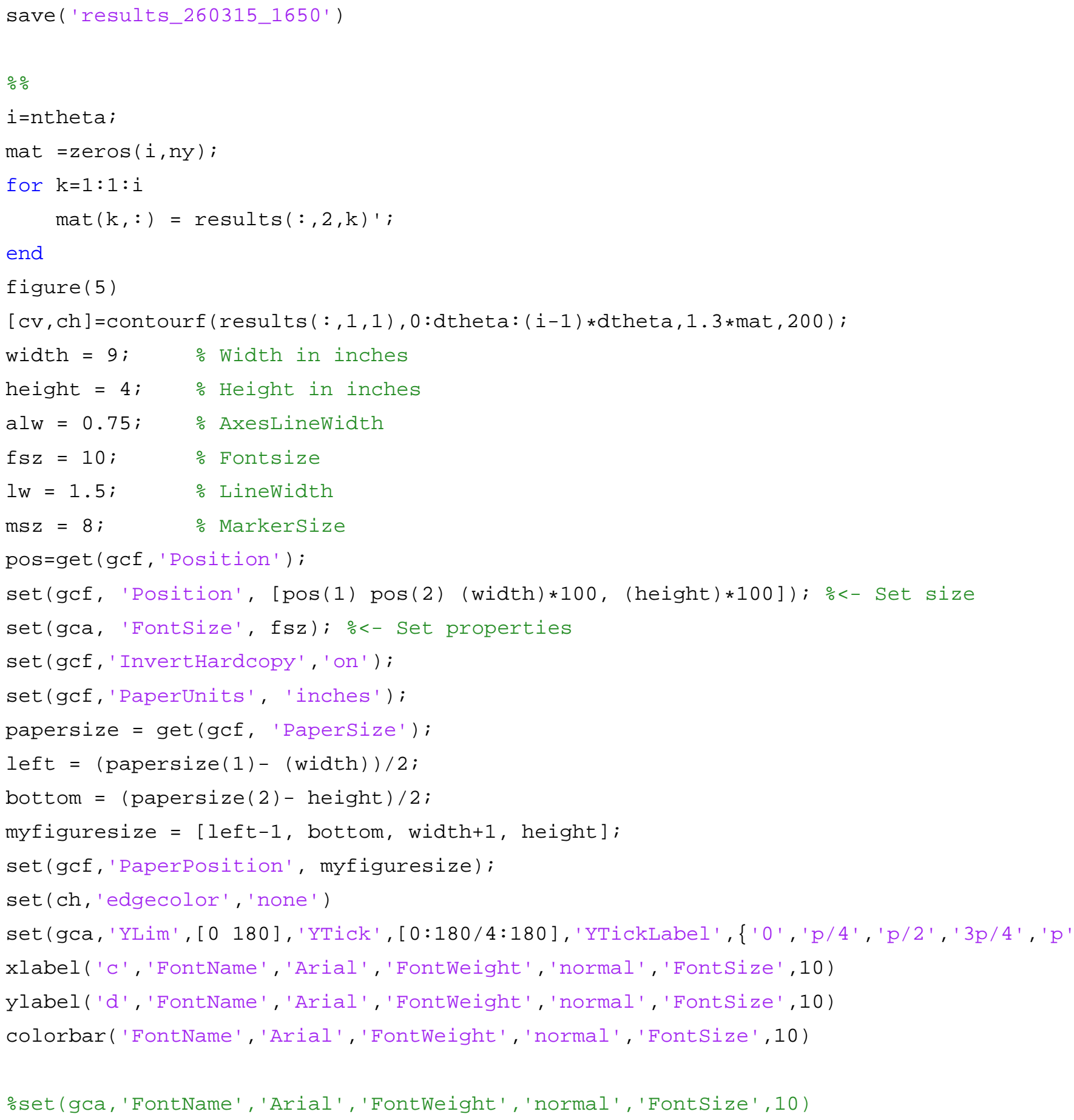

